\title{
SUPERCONGRUENCES FOR RIGID HYPERGEOMETRIC CALABI-YAU THREEFOLDS
}

\author{
LING LONG, FANG-TING TU, NORIKO YUI, WADIM ZUDILIN
}

\begin{abstract}
We establish the supercongruences for the fourteen rigid hypergeometric CalabiYau threefolds over $\mathbb{Q}$ conjectured by Rodriguez-Villegas in 2003. Our first method is based on Dwork's theory of $p$-adic unit roots and it allows us to establish the supercongruences between the truncated hypergeometric series and the corresponding unit roots for ordinary primes. The other method makes use of the theory of hypergeometric motives, in particular, adapts the techniques from the recent work of Beukers, Cohen and Mellit on finite hypergeometric sums over $\mathbb{Q}$. Essential ingredients in executing the both approaches are the modularity of the underlying Calabi-Yau threefolds and a $p$-adic perturbation method applied to hypergeometric functions.
\end{abstract}

\section{INTRODUCTION}

The purpose of this paper is to establish the supercongruences satisfied by fourteen rigid Calabi-Yau threefolds. Each of those threefolds is a particular instance of a one-parameter "hypergeometric" family of Calabi-Yau threefolds - a family whose periods are solutions of the hypergeometric equation with parameters $\boldsymbol{\alpha}=\left\{r_{1}, 1-r_{1}, r_{2}, 1-r_{2}\right\}$ and $\boldsymbol{\beta}=\{1,1,1,1\}$ viewed as multi-sets, where the fourteen possible rational pairs $\left(r_{1}, r_{2}\right)$ are listed in Table 1 below. The corresponding families $V_{\boldsymbol{\alpha}}(\psi)$ are realized as either one-parameter families of hypersurfaces in weighted projective spaces or complete intersections of several such families of hypersurfaces (all explicitly recorded in Tables 3 and 4 below); see [13, 44, 50, 60, for related details. The mirrors of these Calabi-Yau threefolds exist and they are one-parameter families $\hat{\mathcal{V}}_{\boldsymbol{\alpha}}(\lambda)$ of Calabi-Yau threefolds with the Hodge number $h^{2,1}\left(\hat{\mathcal{V}}_{\boldsymbol{\alpha}}(\lambda)\right)=1$ for generic $\lambda$. In all the fourteen cases, the Picard-Fuchs differential equations of $\hat{\mathcal{V}}_{\alpha}(\lambda)$ are precisely the same order 4 hypergeometric differential equations attached to the data $\boldsymbol{\alpha}$ and $\boldsymbol{\beta}$ (see [4 and [16] for more information on this aspect). Their particular analytical solution at the maximally unipotent point - singularity $\lambda=0$ - is given by the hypergeometric function

$$
{ }_{4} F_{3}\left[\begin{array}{l}
\boldsymbol{\alpha} \\
\boldsymbol{\beta}
\end{array} ; \lambda\right]={ }_{4} F_{3}\left[\begin{array}{c}
\alpha_{1}, \alpha_{2}, \alpha_{3}, \alpha_{4} \\
1,1,1
\end{array} ; \lambda:=\sum_{k=0}^{\infty} \frac{\left(\alpha_{1}\right)_{k}\left(\alpha_{2}\right)_{k}\left(\alpha_{3}\right)_{k}\left(\alpha_{4}\right)_{k}}{k !^{4}} \lambda^{k},\right.
$$

Date: $2021 / 10 / 05$

2010 Mathematics Subject Classification. 11F33, 11T24, 14G10, 14J32, 14J33, 33C20.

Key words and phrases. Rigid Calabi-Yau threefold; hypergeometric motive; supercongruence; hypergeometric function; character sum; Picard-Fuchs differential equation; modular form; $p$-adic analysis.

Long is supported by NSF DMS \#1602047 and Tu is supported by a start-up fund at Louisiana State University. Both Long and Tu received travel funding from NSF DMS \#1642598 to attend the MATRIX workshop, Australia. Yui is partially supported by a Discovery Grant from NSERC, Canada. Zudilin was supported by funding from Queen's University to attend BIRS \#16w5009 workshop which initiated this project. Zudilin is partially supported by Laboratory of Mirror Symmetry NRU HSE, RF government grant, ag. no. 14.641.31.0001. 
where $(\alpha)_{k}:=\Gamma(\alpha+k) / \Gamma(\alpha)=\alpha(\alpha+1) \cdots(\alpha+k-1),(\alpha)_{0}:=1$ denotes the Pochhammer symbol (rising factorial) and $\Gamma(x)$ is the standard Gamma function; a general definition of classical hypergeometric functions is given in (28) below.

One of the most known and developed examples is the quintic threefold

$$
V(\psi):=V_{\left\{\frac{1}{5}, \frac{2}{5}, \frac{3}{5}, \frac{4}{5}\right\}}(\psi): \quad X_{1}^{5}+X_{2}^{5}+X_{3}^{5}+X_{4}^{5}+X_{5}^{5}-5 \psi X_{1} X_{2} X_{3} X_{4} X_{5}=0
$$

in $\mathbb{P}^{4}$. For a fixed $\psi$, the equation admits the action of the discrete group

$$
G=\left\{\left(\zeta_{5}^{a_{1}}, \ldots, \zeta_{5}^{a_{5}}\right): a_{1}+\cdots+a_{5} \equiv 0 \bmod 5\right\} \cong(\mathbb{Z} / 5 \mathbb{Z})^{4}
$$

via the map $\left(X_{1}, \ldots, X_{5}\right) \mapsto\left(\zeta_{5}^{a_{1}} X_{1}, \ldots, \zeta_{5}^{a_{5}} X_{5}\right)$, where $\zeta_{5}=e^{2 \pi i / 5}$ is the primitive 5 -th root of unity. Its mirror threefold is constructed from the orbifold $V(\psi) / G$. One way to realize the quotient is letting $y_{j}=X_{j}^{5}$ for $j=1, \ldots, 5, x_{1}=5 \psi X_{1} \cdots X_{5}$ and $\lambda=\psi^{-5}$; the image is

$$
\hat{\mathcal{V}}(\lambda):=\hat{\mathcal{V}}_{\left\{\frac{1}{5}, \frac{2}{5}, \frac{3}{5}, \frac{4}{5}\right\}}(\lambda): \quad y_{1}+\cdots+y_{5}-x_{1}=0, \quad 5^{-5} \lambda x_{1}^{5}=y_{1} \cdots y_{5}
$$

Resolving singularities, one gets a Calabi-Yau threefold $\overline{\hat{\mathcal{V}}(\lambda)}$ with generic $h^{2,1}$ equal to 1 (see [13, 19] for details). By [13], the Picard-Fuchs differential operator of this mirror Calabi-Yau threefold is given by

$$
\theta^{4}-5^{-4} \lambda(5 \theta+1)(5 \theta+2)(5 \theta+3)(5 \theta+4), \quad \text { where } \theta:=\lambda \frac{d}{d \lambda},
$$

whose unique (up to scalar) holomorphic solution near zero is given by the hypergeometric function

$$
\sum_{k=0}^{\infty} \frac{(5 k) !}{k !^{5}}\left(5^{-5} \lambda\right)^{k}={ }_{4} F_{3}\left[\begin{array}{c}
\frac{1}{5}, \frac{2}{5}, \frac{3}{5}, \frac{4}{5} \\
1,1,1
\end{array} ; \lambda .\right.
$$

When $\lambda=1$, the corresponding Calabi-Yau threefold $\overline{\hat{\mathcal{V}}(1)}$ is defined over $\mathbb{Q}$ and it becomes rigid, that is, $h^{2,1}(\overline{\hat{\mathcal{V}}(1)})=0$ meaning that its third Betti number $B_{3}=\operatorname{dim} H^{3}(\overline{\hat{\mathcal{V}}(1)}, \mathbb{C})$ is 2 . It is shown by Schoen [69] that the $\ell$-adic Galois representation (of the absolute Galois group $\left.G_{\mathbb{Q}}:=\operatorname{Gal}(\overline{\mathbb{Q}} / \mathbb{Q})\right)$ arising from étale cohomology $H_{\text {et }}^{3}\left(\overline{\hat{\mathcal{V}}(1)}, \mathbb{Q}_{\ell}\right)$ is modular in the sense that it is isomorphic to the Galois representation attached to a weight 4 level 25 Hecke eigenform $f=f_{\left\{\frac{1}{5}, \frac{2}{5}, \frac{3}{5}, \frac{4}{5}\right\}}$, labeled 25.4.a.b in the database [47]. An expression of $f$ through the Dedekind eta-function $\eta(\tau)=q^{1 / 24} \prod_{n=1}^{\infty}\left(1-q^{n}\right)$, where $q=e^{2 \pi i \tau}$, can be found in Table 1; there we implement the standard notation $\eta_{m}=\eta_{m}(\tau)$ for $\eta(m \tau)$. As shown by Wan in [76], in the ordinary prime case both $V_{\boldsymbol{\alpha}}(1)$ and its mirror $\hat{\mathcal{V}}_{\boldsymbol{\alpha}}(1)$ share the same unit root.

Similar properties hold for the other one-parameter families $V_{\boldsymbol{\alpha}}(\psi)$ listed in Tables 3 and 4 . Their corresponding mirror families $\hat{\mathcal{V}}_{\boldsymbol{\alpha}}(\lambda)$ are provided in Table 5 . When $\lambda=1$, the corresponding mirror fiber $\hat{\hat{V}}_{\boldsymbol{\alpha}}(1)$ happens to be a rigid Calabi-Yau threefold defined over $\mathbb{Q}$, hence its $L$-function computed from the third étale cohomology group is also modular by [22, 30] (see Theorem 5 below). In what follows,

$$
f_{\boldsymbol{\alpha}}(\tau)=\sum_{n=1}^{\infty} a_{n} q^{n}, \quad \text { where } a_{1}=1,
$$

denotes the weight 4 (normalized) modular form corresponding to $\overline{\hat{\mathcal{V}}_{\boldsymbol{\alpha}}(1)}$. 
Based on some numerical evidence, Rodriguez-Villegas came up in [66] with a simple recipe to compute the $p$-th Fourier coefficients $a_{p}=a_{p}\left(f_{\boldsymbol{\alpha}}\right)$ (therefore, all of them) for these fourteen rigid Calabi-Yau threefolds. Proofs of his conjectural (super)congruences are the main result of the paper.

Theorem 1. Let $r_{1}, r_{2} \in\left\{\frac{1}{2}, \frac{1}{3}, \frac{1}{4}, \frac{1}{6}\right\}$ or $\left(r_{1}, r_{2}\right) \in\left\{\left(\frac{1}{5}, \frac{2}{5}\right),\left(\frac{1}{8}, \frac{3}{8}\right),\left(\frac{1}{10}, \frac{3}{10}\right),\left(\frac{1}{12}, \frac{5}{12}\right)\right\}$. Then for each prime $p>5$, the truncated hypergeometric series

$$
{ }_{4} F_{3}\left[\begin{array}{c}
r_{1}, 1-r_{1}, r_{2}, 1-r_{2} ; 1 \\
1,1,1
\end{array}\right]_{p-1}=\sum_{k=0}^{p-1} \frac{\left(r_{1}\right)_{k}\left(1-r_{1}\right)_{k}\left(r_{2}\right)_{k}\left(1-r_{2}\right)_{k}}{k !^{4}}
$$

satisfies

$$
{ }_{4} F_{3}\left[\begin{array}{c}
r_{1}, 1-r_{1}, r_{2}, 1-r_{2} ; 1 \\
1,1,1
\end{array}\right]_{p-1} \equiv a_{p}\left(f_{\left\{r_{1}, 1-r_{1}, r_{2}, 1-r_{2}\right\}}\right) \quad \bmod p^{3} .
$$

The cases $\left(r_{1}, r_{2}\right)=\left(\frac{1}{2}, \frac{1}{2}\right)$ and $\left(\frac{1}{5}, \frac{2}{5}\right)$ have been obtained earlier by Kilbourn [43] and McCarthy [57], respectively. Furthermore, the reduction of case $\left(r_{1}, r_{2}\right)=\left(\frac{1}{2}, \frac{1}{4}\right)$ to Kilbourn's result in [43] has been performed by McCarthy and Fuselier [27]. The remaining eleven cases are new. Our methods provide uniform proofs of all the fourteen hypergeometric cases.

Notice that the Fourier coefficients of $f_{\boldsymbol{\alpha}}$ can be related to the point counting on the corresponding variety over finite fields. A general strategy for this is set up in the recent paper [10] by Beukers, Cohen and Mellit. In particular, the point counting on $\overline{\hat{\mathcal{V}}_{\left\{\frac{1}{5}, \frac{2}{5}, \frac{3}{5}, \frac{4}{5}\right\}}(\lambda)}$ over any finite field of characteristic different from 5 is given explicitly in terms of hypergeometric functions over finite fields, also known as finite hypergeometric functions.

A unifying perspective underneath both classical and finite hypergeometric functions is absorbed by the notion of hypergeometric motives [65, 67, 77]; see [21, 37, 53, 52, 49] for some recent progress and further development of these theme. Postponing details about them to Section 4 , we only indicate that the related hypergeometric motives in our settings are attached to the hypergeometric data

$$
\lambda=1, \quad \boldsymbol{\alpha}=\left\{r_{1}, 1-r_{1}, r_{2}, 1-r_{2}\right\}, \quad \boldsymbol{\beta}=\{1,1,1,1\},
$$

where either $r_{1}, r_{2} \in\left\{\frac{1}{2}, \frac{1}{3}, \frac{1}{4}, \frac{1}{6}\right\}$ or $\left(r_{1}, r_{2}\right) \in\left\{\left(\frac{1}{5}, \frac{2}{5}\right),\left(\frac{1}{8}, \frac{3}{8}\right),\left(\frac{1}{10}, \frac{3}{10}\right),\left(\frac{1}{12}, \frac{5}{12}\right)\right\}$. These choices guarantee that the corresponding motives are all defined over $\mathbb{Q}$. For any prime $p>5$, the corresponding character sum $H_{p}(\boldsymbol{\alpha}, \boldsymbol{\beta} ; 1)$ is explicitly defined in (46) below.

Our next result gives an alternative expression for the Fourier coefficients of Hecke eigenform $f_{\boldsymbol{\alpha}}$ in terms of $H_{p}(\boldsymbol{\alpha}, \boldsymbol{\beta} ; 1)$ and the information about its level.

Theorem 2. Let $p>5$ be a prime and $\boldsymbol{\alpha}$ and $\boldsymbol{\beta}$ as above. Then the following equality holds:

$$
H_{p}(\boldsymbol{\alpha}, \boldsymbol{\beta} ; 1)=a_{p}\left(f_{\boldsymbol{\alpha}}\right)+\chi_{\boldsymbol{\alpha}}(p) \cdot p,
$$

where $a_{p}\left(f_{\boldsymbol{\alpha}}\right)$ is the $p$-th coefficient of the normalized Hecke eigenform and $\chi_{\boldsymbol{\alpha}}$ is a Dirichlet character of order at most 2 , whose precise description is given in Section 3.2. The levels of $f_{\boldsymbol{\alpha}}$ and the characters $\chi_{\boldsymbol{\alpha}}$ are listed in Table 1 (and in Lemma 4 below).

In Table 1, the notation $\chi_{d}$ in the final column stands for the quadratic character $\chi_{d}(p)=$ $\left(\frac{d}{p}\right)$, the Legendre symbol, for unramified $p$; in particular, $\chi_{1}$ denotes the trivial character. In addition, Table 1 records the instances of known formulas for the eigenforms in the second column. 
TABLE 1. The Hecke eigenforms for rigid hypergeometric Calabi-Yau threefolds

\begin{tabular}{|c|c|c|c|c|}
\hline$\left(r_{1}, r_{2}\right)$ & $f_{\boldsymbol{\alpha}}(\tau)$ & level & LMFDB label & $\chi_{\alpha}$ \\
\hline$\left(\frac{1}{2}, \frac{1}{2}\right)$ & $\eta_{2}^{4} \eta_{4}^{4}$ & $8=2^{3}$ & 8.4.a.a & $\chi_{1}$ \\
\hline$\left(\frac{1}{2}, \frac{1}{3}\right)$ & $\eta_{6}^{14} /\left(\eta_{2}^{3} \eta_{18}^{3}\right)-3 \eta_{2}^{3} \eta_{6}^{2} \eta_{18}^{3}$ & $36=2^{2} \cdot 3^{2}$ & $36.4 . a \cdot a$ & $\chi_{3}$ \\
\hline$\left(\frac{1}{2}, \frac{1}{4}\right)$ & $\eta_{4}^{16} /\left(\eta_{2}^{4} \eta_{8}^{4}\right)$ & $16=2^{4}$ & $16.4 . \mathrm{a} . \mathrm{a}$ & $\chi_{2}$ \\
\hline$\left(\frac{1}{2}, \frac{1}{6}\right)$ & $\begin{array}{c}\eta_{4}^{3} \eta_{6}^{6} \eta_{18}^{2} /\left(\eta_{12}^{2} \eta_{36}\right)-3 \eta_{2}^{2} \eta_{6}^{6} \eta_{36}^{3} /\left(\eta_{4} \eta_{12}^{2}\right) \\
\quad+8 \eta_{2}^{3} \eta_{12}^{6} \eta_{36}^{2} /\left(\eta_{6}^{2} \eta_{18}\right)-16 \eta_{12}^{12} / \eta_{6}^{4} \\
\end{array}$ & $72=2^{3} \cdot 3^{2}$ & $72.4 . a \cdot b$ & $\chi_{1}$ \\
\hline$\left(\frac{1}{3}, \frac{1}{3}\right)$ & $\eta_{1}^{3} \eta_{3}^{4} \eta_{9}-27 \eta_{3} \eta_{9}^{4} \eta_{27}^{3}$ & $27=3^{3}$ & $27.4 . a \cdot a$ & $\chi_{1}$ \\
\hline$\left(\frac{1}{3}, \frac{1}{4}\right)$ & $\eta_{3}^{8}$ & $9=3^{2}$ & $9.4 . a \cdot a$ & $\chi_{6}$ \\
\hline$\left(\frac{1}{3}, \frac{1}{6}\right)$ & $\eta_{6}^{10} / \eta_{18}^{2}-27 \eta_{18}^{10} / \eta_{6}^{2}+9 \eta_{6}^{7} \eta_{54}^{3} / \eta_{18}^{2}-9 \eta_{2}^{3} \eta_{18}^{7} / \eta_{6}^{2}$ & $108=2^{2} \cdot 3^{3}$ & $108.4 . \mathrm{a} . \mathrm{a}$ & $\chi_{3}$ \\
\hline$\left(\frac{1}{4}, \frac{1}{4}\right)$ & $\eta_{4}^{10} / \eta_{8}^{2}-8 \eta_{8}^{10} / \eta_{4}^{2}$ & $32=2^{5}$ & $32.4 . a \cdot a$ & $\chi_{1}$ \\
\hline$\left(\frac{1}{4}, \frac{1}{6}\right)$ & $\eta_{12}^{32} /\left(\eta_{6}^{12} \eta_{24}^{12}\right)+16 \eta_{6}^{4} \eta_{24}^{4}$ & $144=2^{4} \cdot 3^{2}$ & 144.4.a.f & $\chi_{2}$ \\
\hline$\left(\frac{1}{6}, \frac{1}{6}\right)$ & & $216=2^{3} \cdot 3^{3}$ & $216.4 . a . c$ & $\chi_{1}$ \\
\hline$\left(\frac{1}{5}, \frac{2}{5}\right)$ & $\eta_{5}^{10} /\left(\eta_{1} \eta_{25}\right)+5 \eta_{1}^{2} \eta_{5}^{4} \eta_{25}^{2}$ & $25=5^{2}$ & $25.4 . a \cdot b$ & $\chi_{5}$ \\
\hline$\left(\frac{1}{8}, \frac{3}{8}\right)$ & $\begin{array}{l}\eta_{1}^{2} \eta_{2} \eta_{4}^{3} \eta_{8}^{3} / \eta_{16}+2 \eta_{2}^{2} \eta_{4}^{3} \eta_{8}^{2} \eta_{16}+8 \eta_{4}^{2} \eta_{8}^{2} \eta_{16}^{2} \eta_{32}^{2}-24 \eta_{4}^{2} \eta_{16}^{2} \eta_{32}^{4} \\
\quad-16 \eta_{2}^{2} \eta_{4} \eta_{8}^{2} \eta_{64}^{4} / \eta_{16}-64 \eta_{8} \eta_{16}^{2} \eta_{32}^{3} \eta_{64}^{2}+32 \eta_{16}^{2} \eta_{32}^{5} \eta_{64}^{2} / \eta_{8} \\
\quad-32 \eta_{4}^{4} \eta_{8} \eta_{32} \eta_{64} \eta_{128}^{2} / \eta_{16}-64 \eta_{8} \eta_{16}^{3} \eta_{32} \eta_{64} \eta_{128}^{2} \\
\quad-256 \eta_{16}^{3} \eta_{32}^{3} \eta_{64} \eta_{128}^{2} / \eta_{8}+128 \eta_{4}^{2} \eta_{32}^{2} \eta_{64}^{3} \eta_{128}^{2} / \eta_{16} \\
\quad-256 \eta_{4}^{4} \eta_{32} \eta_{128}^{4} / \eta_{8}-128 \eta_{16}^{4} \eta_{32} \eta_{128}^{4} / \eta_{8}-512 \eta_{4}^{2} \eta_{64}^{2} \eta_{128}^{4}\end{array}$ & $128=2^{7}$ & $128.4 . a . b$ & $\chi_{2}$ \\
\hline$\left(\frac{1}{10}, \frac{3}{10}\right)$ & & $200=2^{3} \cdot 5^{2}$ & $200.4 . a . f$ & $\chi_{1}$ \\
\hline$\left(\frac{1}{12}, \frac{5}{12}\right)$ & & $864=2^{5} \cdot 3^{3}$ & $864.4 . \mathrm{a} \cdot \mathrm{a}$ & $\chi_{1}$ \\
\hline
\end{tabular}

The supercongruences in Theorem 1 are of interest not only for their own sake; they also happen to encode remarkable symmetries of underlying varieties. In terms of applications, the symmetries can be used to determine the $L$-functions of hypergeometric motives more efficiently.

The paper is organized as follows. In Section 2, we briefly go through historical developments of the supercongruences. Section 3 is for the ordinary case, namely, when the finite sum in (11) does not vanish modulo a prime $p>5$. In this case we show, in Theorem 3 , that the truncated hypergeometric series and Dwork $p$-adic unit root agree modulo $p^{3}$. This is based on Dwork's method and the p-adic perturbation method. En route, a hypergeometric machinery is used to design auxiliary identities - a machinery that naturally complements the $p$-adic perturbation method from [54. We believe this approach is new. Then, in Section 4, hypergeometric motives and finite hypergeometric functions are introduced. Section 5 discuses the fourteen one-parameter families of Calabi-Yau threefolds as well as the associated mirror Calabi-Yau threefolds and corresponding rigid Calabi-Yau threefolds. This section concludes with the proof of Theorem 2. Then our proof of Theorem 1 for all primes $p>5$ is presented in Section [6 building on the results from the previous sections. Importantly, the hypergeometric machinery in Section 3 also gives us a good control of the $p$-adic 
error terms here. Finally, in Section 7 we review our two strategies of proving the supercongruences and indicate some future potential development of the methods. The relation between the Dwork unit roots and varieties $V_{\boldsymbol{\alpha}}(1)$ listed in Table 5 is given in Appendix A via 1-dimensional commutative formal group laws (1-CFGL). In part A.2 we link the cohomology groups of singular Calabi-Yau threefolds (at the conifold point) with their smooth models via Stienstra's work [70, 71]. These relations present interconnections among the $p$ adic unit roots. We demonstrate them at length on one particular example $\left(r_{1}, r_{2}\right)=\left(\frac{1}{2}, \frac{1}{2}\right)$ which corresponds to the complete intersection of four hypersurfaces in a weighted projective space. The other cases are characterized as intersections of two hypersurfaces or as a single hypersurface; they can be computed in a similar manner, and we limit ourselves to listing the logarithms of the corresponding 1-CFGL in Table 6.

We would like to point out that McCarthy previously attempted [58] to prove supercongruences (2), in their full generality, by defining a class of $p$-adic hypergeometric functions which he called $G$-functions.

\section{REVIEW OF RELATED BACKGROUND}

2.1. Supercongruences. In the notation of Section 1, let $\overline{\hat{\mathcal{V}}(1)}$ denote the quintic rigid Calabi-Yau threefold and $f=f_{\left\{\frac{1}{5}, \frac{2}{5}, \frac{3}{5}, \frac{4}{5}\right\}}$ the weight 4 modular form associated with it. One way to see the relation of the $p$-th Fourier coefficient $a_{p}(f)$ to a truncated hypergeometric sum comes from Stienstra's results in [70]. We know from this work that there is a onedimensional formal group arising from $H^{3}\left(V(1), \hat{G}_{m, V(1)}\right)$ whose logarithm is given by

$$
\sum_{n \geq 1} \frac{A_{n}}{n} \tau^{n}=\sum_{n=1}^{\infty}\left(\sum_{k \geq 0}\left(\begin{array}{c}
n-1 \\
5 k
\end{array}\right) \frac{(5 k) !}{k !^{5}}(-5)^{n-1-5 k}\right) \frac{\tau^{n}}{n}
$$

see [70, Theorem 1] for the formula for $A_{n}$. Similar conclusions can be drawn for other thirteen cases using the homogeneous equations listed in Tables 3 and 4 , the recipe is given explicitly in the recent paper [11] by Beukers and Vlasenko, which is an extension of [70]. By the modularity of $\overline{\mathcal{V}(1)}$, this formal group is isomorphic to the formal group whose logarithm is defined by

$$
\sum_{n=1}^{\infty} a_{n}(f) \frac{\tau^{n}}{n}
$$

In particular, when $n=p$ is a prime different from 5 ,

$$
A_{p}=\sum_{k \geq 0}\left(\begin{array}{c}
p-1 \\
5 k
\end{array}\right) \frac{(5 k) !}{k !^{5}}(-5)^{p-1-5 k} \equiv \sum_{k=0}^{p-1} \frac{(5 k) !}{k !^{5}} 5^{-5 k} \bmod p .
$$

From the standard results in commutative formal group laws, it then follows that for each prime $p>5$,

$$
{ }_{4} F_{3}\left[\begin{array}{c}
\frac{1}{5}, \frac{2}{5}, \frac{3}{5}, \frac{4}{5} ; 1 \\
1,1,1
\end{array}\right]_{p-1}=\sum_{k=0}^{p-1} \frac{(5 k) !}{k !^{5}} 5^{-5 k} \equiv a_{p}(f) \quad \bmod p .
$$

Rodriguez-Villegas later noticed and conjectured in [66] that this congruence (and, similarly, the remaining thirteen ones for rigid hypergeometric Calabi-Yau threefolds) hold true modulo $p^{3}$; these are precisely the supercongruences (2) in Theorem 1 . 
The more recent work [64] of Roberts and Rodriguez-Villegas brings to life refined predictions related to (2); it also indicates a heuristics underlying the supercongruences that explains the exponent 3 of the modulus by the Hodge filtration of the corresponding hypergeometric motives. One part of the story is Dwork's unit-root theory in [25], in which the truncated hypergeometric series

$$
F_{s}=F_{s}(\boldsymbol{\alpha}):={ }_{4} F_{3}\left[\begin{array}{c}
r_{1}, 1-r_{1}, r_{2}, 1-r_{2} ; 1 \\
1,1,1
\end{array}\right]_{p^{s}-1} \quad \text { for } s \geq 1
$$

plays an important role. Following Dwork, if $F_{1} \not \equiv 0 \bmod p$, then there exists $\gamma_{p} \in \mathbb{Z}_{p}^{\times}$such that

$$
F_{s} / F_{s-1} \equiv \gamma_{p} \bmod p^{s}
$$

for $s \geq 1$. The claim $F_{s+1} / F_{s} \equiv F_{1} \bmod p^{3}$ for $s=1,2$ (hence for all $s \geq 0$ ) - Theorem 3 below - and its proof form the contents of Section 3. Later in [25], Dwork showed how such unit roots can be used to compute the zeta functions of the fibers on the example of one-parameter deformation of Fermat hypersurfaces. In [79, Theorem 4.3], Yu generalized Dwork's result to Dwork families of Calabi-Yau manifolds, including the quintic family from the Introduction. For the fiber $\psi=1$ of the family, this means that if $F_{1}\left(\left\{\frac{1}{5}, \frac{2}{5}, \frac{3}{5}, \frac{4}{5}\right\}\right) \not \equiv 0$ $\bmod p$, then $\gamma_{p}$ defined in (17) happens to be a reciprocal root of the zeta function of $V(1)$ which is invertible in $\mathbb{Z}_{p}$. Using the construction of the quintic mirror, it is known that it is also the reciprocal root of the zeta function of $\overline{\hat{V}}_{\boldsymbol{\alpha}}(1)$ which is invertible in $\mathbb{Z}_{p}$ (see [14] by Candelas, de la Ossa and Rodriguez-Villegas).

2.2. $p$-adic perturbation method. Among a variety of techniques proving supercongruences for truncated hypergeometric sums, the most relevant one is the so-called $p$-adic perturbation method described in [54] and originated in [15, 51]. It is efficient in dealing with entities that can be represented through Gamma values. In plain language, the method breaks down the entities into two parts, one in terms of the $p$-adic Gamma functions $\Gamma_{p}(x)$ that have good local analytic property and the other one that collects all $p$-powers. (We will use this strategy in our later discussion.) As a consequence, we can reduce a desired supercongruence to a major term and error terms. In the final stage, eliminations of the error terms are often done through known hypergeometric identities, which are perturbed $p$-adically using additional inputs like Galois symmetries. This approach was later used in [20, 73].

2.3. Hidden symmetries. Supercongruences often seem to highlight some hidden symmetries typically appearing as classical hypergeometric identities, symmetries that are crucial to efficiency of the $p$-adic perturbation method. For example, Ahlgren and Ono [3] used a "hidden symmetry" identity

$$
\sum_{k=1}^{n}\left(\begin{array}{c}
n+k \\
k
\end{array}\right)^{2}\left(\begin{array}{l}
n \\
k
\end{array}\right)^{2}\left(1+2 k H_{n+k}+2 k H_{n-k}-4 k H_{k}\right)=0
$$

to establish the following supercongruence of the Apéry numbers: for any prime $p>2$,

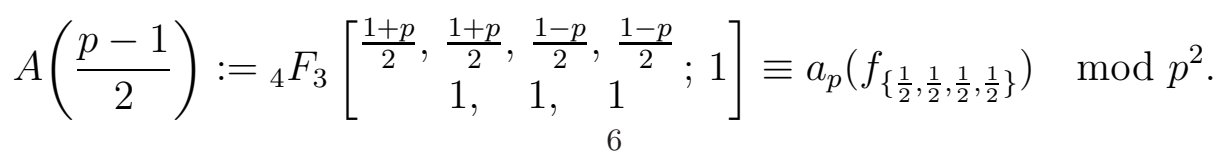


Here $f_{\left\{\frac{1}{2}, \frac{1}{2}, \frac{1}{2}, \frac{1}{2}\right\}}(\tau)=\eta(2 \tau)^{4} \eta(4 \tau)^{4}$ (as in Table 1) and

$$
H_{k}:=\sum_{j=1}^{k} \frac{1}{j}
$$

denotes the $k$-th partial harmonic sum, with $H_{0}=0$. As shown in [9], the formal group method leads to the congruence (9) modulo $p$ only; identity (8) extends it modulo $p^{2}$. This latter identity (8) was verified by a clever execution of the Wilf-Zeiberger method of creative telescoping, though, in fact, it is a rather elementary analysis of the residue sum for the rational function

$$
\frac{\prod_{j=1}^{n}(t-j)^{2}}{\prod_{j=0}^{n}(t+j)^{2}}
$$

(see [83] and also [56], 61, Lemma 5.1]). Extending the work [3], Kilbourn [43] demonstrated that, for primes $p>2$,

$$
{ }_{4} F_{3}\left[\begin{array}{c}
\frac{1}{2}, \frac{1}{2}, \frac{1}{2}, \frac{1}{2} ; 1 \\
1,1,1
\end{array}\right]_{p-1} \equiv a_{p}\left(f_{\left\{\frac{1}{2}, \frac{1}{2}, \frac{1}{2}, \frac{1}{2}\right\}}\right) \quad \bmod p^{3},
$$

a supercongruence originally conjectured by van Hamme in [74. The later development of the method towards proving some other instances of (2) was undertaken in [27, 57].

Quite remarkably, a somewhat simpler companion identity associated to (86) exists,

$$
\sum_{k=0}^{n}\left(\begin{array}{c}
n+k \\
k
\end{array}\right)^{2}\left(\begin{array}{l}
n \\
k
\end{array}\right)^{2}\left(H_{n+k}+H_{n-k}-2 H_{k}\right)=0,
$$

that possesses the same (if not simpler) proof [83]. With the help of (12), we can deduce the supercongruence (11) for ordinary primes $p$ without using a heavy machinery of character sums - somewhat more straightforward than [43, 57]. The next section details the related approach and gives a generalization of (12), which on addition of $p$-adic perturbation terms is capable of treating all the cases in Theorem 1 for ordinary primes.

\section{DWORK'S APPROACH FOR ORDINARY PRIMES}

3.1. $p$-adic setup. Dwork [25] laid down a framework for $p$-adic hypergeometric functions, which we discuss here in relation to the objectives of this paper. In particular, we show in Theorem 3 below that for any ordinary good prime $p$ the finite sum (1) agrees with the corresponding Dwork unit root modulo $p^{3}$.

We use $\lfloor x\rfloor$ for the floor function of $x \in \mathbb{R}$ and denote $\{x\}:=x-\lfloor x\rfloor$ the fractional part. For the discussion in this section, we work over the ring of $p$-adic integers $\mathbb{Z}_{p}$ with $p>5$ being any fixed prime; see [18] for more detailed information on $\mathbb{Z}_{p}$ and the $p$-adic Gamma function $\Gamma_{p}(x)$. Furthermore, for $r \in \mathbb{Z}_{p}$, let $[r]_{0}$ denote its first $p$-adic digit.

Lemma 1. Given an integer $k, 0 \leq k<p$, and $r \in \mathbb{Z}_{p}^{\times}$, the rising factorial $(r)_{k}$ is in $\mathbb{Z}_{p}^{\times}$if and only if $k \leq[-r]_{0}$.

Proof. This follows from $(r)_{k}=r(r+1) \cdots(r+k-1)$ and the definition of $[-r]_{0}$.

The following discussion is based on Dwork's work on $p$-adic cycles [25]. We will use Dwork's dash operation - the map $\mathbb{Q} \cap \mathbb{Z}_{p} \rightarrow \mathbb{Q} \cap \mathbb{Z}_{p}$ defined by

$$
r^{\prime}=\left(r+[-r]_{0}\right) / p
$$


which despite of its notational appearance has nothing to do with the usual derivative or derivations. It is easy to see that $p r^{\prime}-r=[-r]_{0} \in\{0,1, \ldots, p-1\}$. If we write $r=\ell / d$ with $\operatorname{gcd}(\ell, d)=1$, the $p$-adic integer $r^{\prime}$ is $\tilde{\ell} / d$ with $\tilde{\ell} \equiv \ell / p \bmod d$. For each multi-set $\boldsymbol{\alpha}=\left\{r_{1}, r_{2}, r_{3}, r_{4}\right\}=\left\{r_{1}, r_{2}, 1-r_{1}, 1-r_{2}\right\}$ in Table1 and a prime $p$ not dividing the common denominator of $r_{1}, r_{2}$, the multi-set $\left\{r_{1}^{\prime}, r_{2}^{\prime}, r_{3}^{\prime}, r_{4}^{\prime}\right\}$ is just a permutation of $\left\{r_{1}, r_{2}, r_{3}, r_{4}\right\}$ (we will cast this property of $\boldsymbol{\alpha}$ as the closedness under the Galois conjugation in Section 44). In particular, the dash operation preserves any of our fourteen multi-sets $\boldsymbol{\alpha}$ for every prime $p>5$.

Proposition 1 (Dwork). Given a prime $p$ and a multi-set $\boldsymbol{\alpha}=\left\{r_{1}, r_{2}, r_{3}, r_{4}\right\}$ with $r_{1}, r_{2} \in$ $(0,1) \cap \mathbb{Z}_{p}^{\times}$such that $\boldsymbol{\alpha}$ is preserved by the dash operation, for any integers $s \geq t \geq 1$ we have $F_{s} F_{t-1} \equiv F_{t} F_{s-1} \bmod p^{t}$, where $F_{s}=F_{s}(\boldsymbol{\alpha})$ is defined in (6) $)$. In particular, if $F_{1}(\boldsymbol{\alpha}) \not \equiv 0$ $\bmod p$, then there exists a unique $\gamma_{p}=\gamma_{p}(\boldsymbol{\alpha}) \in \mathbb{Z}_{p}^{\times}$such that for any integer $s \geq 1$,

$$
F_{s} / F_{s-1} \equiv \gamma_{p} \bmod p^{s}
$$

Proof. Here we indicate how to deduce the proposition from the results in [25]. Denote $A(n)=\left(\prod_{i=1}^{4}\left(r_{i}\right)_{n}\right) / n !^{4}$. Since $\boldsymbol{\alpha}$ is closed under the dash operator, by [25, Corollary 2] we deduce that $A(n) / A(\lfloor n / p\rfloor) \in \mathbb{Z}_{p}$ and for all integers $m, s \geq 1$,

$$
\frac{A(n)}{A(\lfloor n / p\rfloor)} \equiv \frac{A\left(n+m p^{s+1}\right)}{A\left(\lfloor n / p\rfloor+m p^{s}\right)} \bmod p^{s+1} .
$$

This property and [25, Theorems 2,3] imply that $B^{(i)}(n)=A(n)$ for all $i=1,2, \ldots$, and [25, Equation $\left.\left(3.2^{\prime}\right)\right]$ at $x=1$ becomes equivalent to the claim $F_{s} F_{t-1} \equiv F_{t} F_{s-1} \bmod p^{t}$ for integers $s \geq t \geq 1$. This implies the existense of the $p$-adic limit $\gamma_{p}$ of $F_{s} / F_{s-1}$ as $s \rightarrow \infty$ when $F_{1} \not \equiv 0(\bmod p)$; in other words, when $p$ is an ordinary prime. The limit $\gamma_{p}$ is a unit in $\mathbb{Z}_{p}$, because $\gamma_{p} \equiv F_{1}(\bmod p)$.

Theorem 3. Let $\boldsymbol{\alpha}=\left\{r_{1}, r_{2}, 1-r_{1}, 1-r_{2}\right\}$ be one of the fourteen multi-sets from Table 1 and $p$ a prime such that $r_{1}, r_{2} \in \mathbb{Z}_{p}^{\times}$. Then for any integer $s \geq 1$,

$$
F_{s+1}(\boldsymbol{\alpha}) \equiv F_{s}(\boldsymbol{\alpha}) F_{1}(\boldsymbol{\alpha}) \bmod p^{3} .
$$

We will require some preparation before proving Theorem 3 . The following reduction of a quotient of rising factorials makes use of the relation between the Gamma and $p$-adic Gamma functions: for prime $p>2$ and a positive integer $n$,

$$
\Gamma(n)=(-1)^{n} \Gamma_{p}(n)\left\lfloor\frac{n-1}{p}\right\rfloor ! p^{\lfloor(n-1) / p\rfloor} .
$$

Lemma 2. Let $k \in \mathbb{Z}_{\geq 0}, a=[k]_{0}$ and $b=(k-a) / p$, that $i s, k=a+b p$. Then for any $r \in \mathbb{Z}_{p}^{\times}$,

$$
\frac{(r)_{k}}{(1)_{k}}=\frac{-\Gamma_{p}(r+k)}{\Gamma_{p}(1+k) \Gamma_{p}(r)} \frac{\left(r^{\prime}\right)_{b}}{(1)_{b}} \cdot\left(\left(r^{\prime}+b\right) p\right)^{\nu\left(a,[-r]_{0}\right)}
$$

where

$$
\nu(a, x)=-\left\lfloor\frac{x-a}{p-1}\right\rfloor_{8}= \begin{cases}0 & \text { if } a \leq x, \\ 1 & \text { if } x<a<p .\end{cases}
$$


Proof. Write $r \in \mathbb{Z}_{p}^{\times}$as $r=[r]_{0}+p h$. Then $[-r]_{0}=p-[r]_{0}$ and $r^{\prime}=h+1$. First, assume that $r$ is a positive integer. Then from (13) we deduce that

$$
\frac{(r)_{k}}{(1)_{k}}=\frac{\Gamma(r+k)}{\Gamma(1+k) \Gamma(r)}=\frac{-\Gamma_{p}(r+k)}{\Gamma_{p}(1+k) \Gamma_{p}(r)} \frac{\lfloor(r+k-1) / p\rfloor !}{\lfloor k / p\rfloor !\lfloor(r-1) / p\rfloor !} p^{\lfloor(r+k-1) / p\rfloor-\lfloor k / p\rfloor-\lfloor(r-1) / p\rfloor} .
$$

Since $r+k=a+[r]_{0}+p(h+b)$, we have

$$
\left\lfloor\frac{r+k-1}{p}\right\rfloor=h+b+ \begin{cases}0 & \text { if } a \leq p-[r]_{0}=[-r]_{0} \\ 1 & \text { if }[-r]_{0}<a<p\end{cases}
$$

so that for $0<a \leq[-r]_{0}$,

$$
\frac{\lfloor(r+k-1) / p\rfloor !}{\lfloor k / p\rfloor !\lfloor(r-1) / p\rfloor !} p^{\lfloor(r+k-1) / p\rfloor-\lfloor k / p\rfloor-\lfloor(r-1) / p\rfloor}=\frac{(h+b) !}{b ! h !}=\frac{(h+1)_{b}}{(1)_{b}}=\frac{\left(r^{\prime}\right)_{b}}{(1)_{b}},
$$

and for $[-r]_{0}<a<p$,

$$
\frac{\lfloor(r+k-1) / p\rfloor !}{\lfloor k / p\rfloor !\lfloor(r-1) / p\rfloor !} p^{\lfloor(r+k-1) / p\rfloor-\lfloor k / p\rfloor-\lfloor(r-1) / p\rfloor}=\frac{(h+b) !}{b ! h !}(h+b+1) p=\frac{\left(r^{\prime}\right)_{b}}{(1)_{b}}\left(r^{\prime}+b\right) p .
$$

Therefore,

$$
\frac{(r)_{k}}{(1)_{k}}=\frac{-\Gamma_{p}(r+k)}{\Gamma_{p}(1+k) \Gamma_{p}(r)} \frac{\left(r^{\prime}\right)_{b}}{(1)_{b}}\left(\left(r^{\prime}+b\right) p\right)^{\nu\left(a,[-r]_{0}\right)} .
$$

The identity holds for all $r \in \mathbb{Z}_{p}^{\times}$by the continuity of the $p$-adic Gamma function.

We complement Lemma 2 by its particular instance $k=a$ (so that $b=0$ ):

$$
\frac{-\Gamma_{p}(r+a)}{\Gamma_{p}(1+a) \Gamma_{p}(r)}=\frac{(r)_{a}}{a !} \cdot \frac{1}{\left(r^{\prime} p\right)^{\nu\left(a,[-r]_{0}\right)}}
$$

which in turn implies the following for $k=a+b p$ :

$$
\begin{aligned}
\frac{(r)_{a+b p}}{(1)_{a+b p}} & =\frac{-\Gamma_{p}(r+a)}{\Gamma_{p}(1+a) \Gamma_{p}(r)} \frac{\left(r^{\prime}\right)_{b}}{(1)_{b}}\left(\left(r^{\prime}+b\right) p\right)^{\nu\left(a,[-r]_{0}\right)} \frac{\Gamma_{p}((r+a)+b p) \Gamma_{p}(1+a)}{\Gamma_{p}(r+a) \Gamma_{p}((1+a)+b p)} \\
& =\frac{(r)_{a}}{a !} \frac{\left(r^{\prime}\right)_{b}}{(1)_{b}}\left(1+\frac{b}{r^{\prime}}\right)^{\nu\left(a,[-r]_{0}\right)} \frac{\Gamma_{p}((r+a)+b p) \Gamma_{p}(1+a)}{\Gamma_{p}(r+a) \Gamma_{p}((1+a)+b p)} .
\end{aligned}
$$

This "key reduction" formula (16) is instrumental in separating the $p$-adic terms in a way convenient to our future analysis.

We will use the following local analytic properties of the $p$-adic Gamma function (see for example [54]). For $t, s \in \mathbb{Z}_{p}$,

$$
\Gamma_{p}(t+s p)=\Gamma_{p}(t)\left(1+s p G_{1}(t)+\frac{(s p)^{2}}{2} G_{2}(t)\right) \quad \bmod p^{3},
$$

where

From definition,

$$
G_{1}(t):=\frac{\mathrm{d}}{\mathrm{d} t} \log \Gamma_{p}(t)=\frac{\mathrm{d} \Gamma_{p}(t) / \mathrm{d} t}{\Gamma_{p}(t)} \quad \text { and } \quad G_{2}(t):=\frac{\mathrm{d}^{2} \Gamma_{p}(t) / \mathrm{d} t^{2}}{\Gamma_{p}(t)} .
$$

$$
\frac{\mathrm{d}}{\mathrm{d} t} G_{1}(t)=G_{2}(t)-G_{1}(t)^{2}
$$


Lemma 3. For any $t \in \mathbb{Z}_{p}$ and an integer $a \in\{0,1, \ldots, p-1\}$, we have

$$
\begin{aligned}
\frac{\mathrm{d}}{\mathrm{d} t}(t)_{a}=(t)_{a} & \left(G_{1}(t+a)-G_{1}(t)+\frac{\nu\left(a,[-t]_{0}\right)}{t+[-t]_{0}}\right) ; \\
\frac{\mathrm{d}^{2}}{\mathrm{~d} t^{2}}(t)_{a}=(t)_{a} & \left(\left(G_{1}(t+a)-G_{1}(t)+\frac{\nu\left(a,[-t]_{0}\right)}{t+[-t]_{0}}\right)^{2}\right. \\
& \left.+G_{2}(t+a)-G_{2}(t)-G_{1}(t+a)^{2}+G_{1}(t)^{2}-\frac{\nu\left(a,[-t]_{0}\right)}{\left(t+[-t]_{0}\right)^{2}}\right),
\end{aligned}
$$

where $\nu(a, x)$ is defined in (14). Notice that $t+[-t]_{0}=p t^{\prime}$.

Proof. By the functional equation of the $p$-adic Gamma function, for $0 \leq a<p$ we have

$$
(t)_{a}=t(t+1) \cdots(t+a-1)=(-1)^{a} \frac{\Gamma_{p}(t+a)}{\Gamma_{p}(t)}\left(t+[-t]_{0}\right)^{\nu\left(a,[-t]_{0}\right)} .
$$

Logarithmically differentiating the equality we derive the required claims.

Let $\boldsymbol{\alpha}=\left\{r_{1}, r_{2}, r_{3}, r_{4}\right\}=\left\{r_{1}, r_{2}, 1-r_{1}, 1-r_{2}\right\}$ be one of the fourteen multi-sets from Table 1 and $p$ a prime such that $r_{1}, r_{2} \in \mathbb{Z}_{p}^{\times}$. The dash operation preserves the multi-set; from now on we will numerate the entries in the multi-set $\left\{r_{1}, r_{2}, r_{3}, r_{4}\right\}$ in such a way that

$$
r_{1}^{\prime} \leq r_{2}^{\prime} \leq r_{3}^{\prime} \leq r_{4}^{\prime}
$$

This inequality, the structure of the entries in $\left\{r_{1}, r_{2}, r_{3}, r_{4}\right\}=\left\{r_{1}^{\prime}, r_{2}^{\prime}, r_{3}^{\prime}, r_{4}^{\prime}\right\}$ and the trivial property $(1-r)^{\prime}=1-r^{\prime}$ for rational $r \in(0,1) \cap \mathbb{Z}_{p}^{\times}$result in

$$
r_{1}+r_{4}=r_{2}+r_{3}=1 \text { and } r_{1}^{\prime}+r_{4}^{\prime}=r_{2}^{\prime}+r_{3}^{\prime}=1 \text {. }
$$

Furthermore, denote

$$
a_{j}:=\left[-r_{j}\right]_{0}=p r_{j}^{\prime}-r_{j}, \quad \text { for } j=1,2,3,4 .
$$

From the ordering chosen in (19), if $i<j$ then $a_{i}-a_{j}=p\left(r_{i}^{\prime}-r_{j}^{\prime}\right)-\left(r_{i}-r_{j}\right) \leq-\left(r_{i}-r_{j}\right)<1$, hence $a_{i} \leq a_{j}$ as they are both integers. Putting together,

$$
a_{1} \leq a_{2} \leq a_{3} \leq a_{4} \quad \text { and } \quad a_{1}+a_{4}=a_{2}+a_{3}=p-1 .
$$

The extra factors appearing in (16) are collected in the expression

$$
\Lambda_{\alpha}(a+b p):=\prod_{j=1}^{4}\left(1+\frac{b}{r_{j}^{\prime}}\right)^{\nu\left(a,\left[-r_{j}\right]_{0}\right)}= \begin{cases}1 & \text { if } 0 \leq a \leq a_{1} \\ \left(1+b / r_{1}^{\prime}\right) & \text { if } a_{1}<a \leq a_{2} \\ \left(1+b / r_{1}^{\prime}\right)\left(1+b / r_{2}^{\prime}\right) & \text { if } a_{2}<a \leq a_{3}\end{cases}
$$

(we omit the other cases in view of their irrelevance) and, for $0 \leq a<p$, the $p$-adic order of the Pochhammer quotient

$$
\frac{\prod_{j=1}^{4}\left(r_{j}\right)_{a}}{a !^{4}}
$$

is equal to $s \in\{0,1,2,3,4\}$ if and only if $a_{s}<a \leq a_{s+1}$, where we additionally set $a_{0}=-1$ and $a_{5}=p-1$.

It follows from (17) that

$$
\frac{\prod_{j=1}^{4} \Gamma_{p}\left(\left(r_{j}+a\right)+b p\right)}{\Gamma_{p}((1+a)+b p)^{4}} \equiv \frac{\prod_{j=1}^{4} \Gamma_{p}\left(r_{j}+a\right)}{\Gamma_{p}(1+a)^{4}}\left(1+J_{1}(a) b p+J_{2}(a)(b p)^{2}\right) \quad \bmod p^{3},
$$


where the coefficients $J_{1}(a)$ and $J_{2}(a)$ are given by

$$
\begin{aligned}
J_{1}(a)=J_{1}(a, \boldsymbol{\alpha}):= & \sum_{j=1}^{4}\left(G_{1}\left(r_{j}+a\right)-G_{1}(1+a)\right) \\
J_{2}(a)=J_{2}(a, \boldsymbol{\alpha}):= & 10 G_{1}(1+a)^{2}-4 G_{1}(1+a) \sum_{j=1}^{4} G_{1}\left(r_{j}+a\right) \\
& +\sum_{1 \leq j<\ell \leq 4} G_{1}\left(r_{j}+a\right) G_{1}\left(r_{\ell}+a\right)+\frac{1}{2} \sum_{j=1}^{4}\left(G_{2}\left(r_{j}+a\right)-G_{2}(1+a)\right) .
\end{aligned}
$$

By the key reduction formula (16) and from (22),

$$
\begin{aligned}
F_{s+1}(\boldsymbol{\alpha})= & \sum_{a=0}^{p-1} \sum_{b=0}^{p^{s}-1} \frac{\prod_{j=1}^{4}\left(r_{j}\right)_{a+b p}}{(1)_{a+b p}^{4}} \\
\equiv & \sum_{b=0}^{p^{s}-1} \frac{\prod_{j=1}^{4}\left(r_{j}^{\prime}\right)_{b}}{b !^{4}} \sum_{a=0}^{p-1} \frac{\prod_{j=1}^{4}\left(r_{j}\right)_{a}}{a !^{4}} \\
& \quad \times \Lambda_{\boldsymbol{\alpha}}(a+b p)\left(1+J_{1}(a) \cdot b p+J_{2}(a) \cdot(b p)^{2}\right) \bmod p^{3} .
\end{aligned}
$$

3.2. Dirichlet characters. For $r \in \mathbb{Z}_{p}^{\times}$, the reflection formula of the $p$-adic Gamma function reads $\Gamma_{p}(r) \Gamma_{p}(1-r)=(-1)^{[r]_{0}}$. In our situation $\left[r_{j}\right]_{0}=p-\left[-r_{j}\right]_{0} \equiv a_{j}(\bmod 2)$ for $j=1,2,3,4$ and $p>5$, therefore

$$
\chi_{\boldsymbol{\alpha}}(p):=\prod_{j=1}^{4} \Gamma_{p}\left(r_{j}\right)=\Gamma_{p}\left(r_{1}\right) \Gamma_{p}\left(1-r_{1}\right) \Gamma_{p}\left(r_{2}\right) \Gamma_{p}\left(1-r_{2}\right)=(-1)^{a_{1}+a_{2}} .
$$

We will use the result in our further derivations.

Lemma 4. For each multi-set $\boldsymbol{\alpha}=\left\{r_{1}, 1-r_{1}, r_{2}, 1-r_{2}\right\}$ as in Theorem 1, $\chi_{\boldsymbol{\alpha}}$ defines the quadratic Dirichlet character as in the last column of Table 1 .

Proof. It is straightforward that $\Gamma_{p}(r) \Gamma_{p}(1-r)$ agrees with the Legendre symbol $\left(\frac{-1}{p}\right)$, $\left(\frac{-3}{p}\right),\left(\frac{-2}{p}\right)$ and $\left(\frac{-1}{p}\right)$ when $r=\frac{1}{2}, \frac{1}{3}, \frac{1}{4}, \frac{1}{6}$, respectively. Furthermore, a direct verification implies that the right-hand side of (25) is 1 when $\left(r_{1}, r_{2}\right)=\left(\frac{1}{10}, \frac{3}{10}\right)$ or $\left(\frac{1}{12}, \frac{5}{12}\right)$; $\left(\frac{5}{p}\right)$ when $\left(r_{1}, r_{2}\right)=\left(\frac{1}{5}, \frac{2}{5}\right) ;$ and $\left(\frac{2}{p}\right)$ when $\left(r_{1}, r_{2}\right)=\left(\frac{1}{8}, \frac{3}{8}\right)$.

3.3. Proof of Theorem 3. In view of (24), Theorem 3 is implied by the following lemma.

Lemma 5. For any $b \in \mathbb{Z}_{\geq 0}$, the congruence

$$
\sum_{a=0}^{p-1} \frac{\prod_{j=1}^{4}\left(r_{j}\right)_{a}}{a !^{4}}\left(\Lambda(a+b p)\left(1+J_{1}(a) \cdot b p+J_{2}(a) \cdot(b p)^{2}\right)-1\right) \equiv 0 \bmod p^{3}
$$

holds. 
Proof. From the $p$-adic evaluation of (21) and the definition of $\Lambda(a+b p)$, we conclude that the left-hand side modulo $p^{3}$ is a quadratic polynomial $C_{0}+C_{1} b+C_{2} b^{2}$ in $b$, with the constant term $C_{0}=0$, and

$$
\begin{aligned}
C_{1}=p & \sum_{a=0}^{a_{1}} \frac{\prod_{j=1}^{4}\left(r_{j}\right)_{a}}{a !^{4}} J_{1}(a)+\sum_{a=a_{1}+1}^{a_{2}} \frac{\prod_{j=1}^{4}\left(r_{j}\right)_{a}}{a !^{4}}\left(\frac{1}{r_{1}^{\prime}}+p J_{1}(a)\right) \\
& +\left(\frac{1}{r_{1}^{\prime}}+\frac{1}{r_{2}^{\prime}}\right) \sum_{a=a_{2}+1}^{a_{3}} \frac{\prod_{j=1}^{4}\left(r_{j}\right)_{a}}{a !^{4}} ; \\
C_{2}=p^{2} & \sum_{a=0}^{a_{1}} \frac{\prod_{j=1}^{4}\left(r_{j}\right)_{a}}{a !^{4}} J_{2}(a)+\frac{p}{r_{1}^{\prime}} \sum_{a=a_{1}+1}^{a_{2}} \frac{\prod_{j=1}^{4}\left(r_{j}\right)_{a}}{a !^{4}} J_{1}(a) \\
& +\frac{1}{r_{1}^{\prime} r_{2}^{\prime}} \sum_{a=a_{2}+1}^{a_{3}} \frac{\prod_{j=1}^{4}\left(r_{j}\right)_{a}}{a !^{4}}
\end{aligned}
$$

where the terms, which are zero modulo $p^{3}$ for trivial reasons, are discarded. Our goal is to demonstrate that $C_{1} \equiv 0 \bmod p^{3}$ and $C_{2} \equiv 0 \bmod p^{3}$.

Introduce the rational function

$$
R(t)=\frac{\prod_{j=1}^{4} \prod_{i=1}^{a_{j}}\left(t-i+p r_{j}^{\prime}\right)}{\prod_{i=0}^{p-1}(t+i)^{2}} .
$$

This function is a generalization of the one in (10), with the correction terms $p r_{j}^{\prime}$ added to make our argument below more efficient. The degree of its numerator $a_{1}+a_{2}+a_{3}+a_{4}=$ $\left(a_{1}+a_{4}\right)+\left(a_{2}+a_{3}\right)=(p-1)+(p-1)=2(p-1)$ is by 2 less than the degree $2 p$ of its denominator; hence it can be represented as the sum of partial fractions,

$$
R(t)=\sum_{k=0}^{p-1}\left(\frac{A_{k}}{(t+k)^{2}}+\frac{B_{k}}{t+k}\right)
$$

for which the identity

$$
\sum_{k=0}^{p-1} B_{k}=\sum_{k=0}^{p-1} \operatorname{Res}_{t=-k} R(t)=-\operatorname{Res}_{t=\infty} R(t)=0
$$

is implied by the residue sum theorem. The coefficients in the partial-fraction decomposition can be computed explicitly:

$$
\begin{aligned}
& A_{k}=\left.R(t)(t+k)^{2}\right|_{t=-k}=\frac{\prod_{j=1}^{4} \prod_{i=1}^{a_{j}}\left(k+i-p r_{j}^{\prime}\right)}{k !^{2}(p-1-k) !^{2}}=\frac{\prod_{j=1}^{4}\left(k+1-p r_{j}^{\prime}\right)_{a_{j}}}{(1)_{k}^{2}(1)_{p-1-k}^{2}} \\
& B_{k}=A_{k}\left(-\sum_{j=1}^{4} \sum_{i=1}^{a_{j}} \frac{1}{k+i-p r_{j}^{\prime}}+2 H_{k}-2 H_{p-1-k}\right)
\end{aligned}
$$


where as before $H_{k}$ stands for the $k$-th partial harmonic sum. Using equation (18) and the similar transformation

$$
\begin{aligned}
(k+1-p \lambda)_{a} & =(-1)^{a} \frac{\Gamma_{p}(k+1+a-p \lambda)}{\Gamma_{p}(k+1-p \lambda)}\left(k+1-p \lambda+[-(k+1-p \lambda)]_{0}\right)^{\nu\left(a,[-k-1]_{0}\right)} \\
& =(-1)^{a} \frac{\Gamma_{p}(k+1+a-p \lambda)}{\Gamma_{p}(k+1-p \lambda)}(p(1-\lambda))^{\nu\left(a,[-k-1]_{0}\right)}
\end{aligned}
$$

for $0 \leq k \leq p-1$ and $0<a<p$, as well as noticing that

$$
\nu\left(a_{j},[-k-1]_{0}\right)=\nu\left(k+1,\left[-a_{j}\right]_{0}\right)=\nu\left(k+1,\left[r_{j}\right]_{0}\right)
$$

for $j=1,2,3,4$, we find out that

$$
\begin{aligned}
A_{k} & =\frac{\prod_{j=1}^{4} \Gamma_{p}\left(k+1+a_{j}-p r_{j}^{\prime}\right)\left(p\left(1-r_{j}^{\prime}\right)\right)^{\nu\left(a_{j},[-k-1]_{0}\right)}}{\Gamma_{p}(k+1)^{2} \Gamma_{p}(p-k)^{2} \prod_{j=1}^{4} \Gamma_{p}\left(k+1-p r_{j}^{\prime}\right)} \\
& =\frac{\Gamma_{p}(k+1-p)^{2} \prod_{j=1}^{4} \Gamma_{p}\left(k+1-r_{j}\right)\left(p\left(1-r_{j}^{\prime}\right)\right)^{\nu\left(k+1,\left[r_{j}\right]_{0}\right)}}{\Gamma_{p}(k+1)^{2} \prod_{j=1}^{4} \Gamma_{p}\left(k+1-p r_{j}^{\prime}\right)} \\
& =\frac{\prod_{j=1}^{4}\left(-r_{j}\right)_{k+1}}{k !^{4}} \frac{\Gamma_{p}(k+1)^{2} \Gamma_{p}(k+1-p)^{2} \prod_{j=1}^{4} \Gamma_{p}\left(-r_{j}\right)}{\prod_{j=1}^{4} \Gamma_{p}\left(k+1-p r_{j}^{\prime}\right)} \\
& =\frac{r_{1} r_{2} r_{3} r_{4} \prod_{j=1}^{4}\left(1-r_{j}\right)_{k}}{k !^{4}} \frac{\Gamma_{p}(k+1)^{2} \Gamma_{p}(k+1-p)^{2} \prod_{j=1}^{4} \Gamma_{p}\left(1-r_{j}\right)}{r_{1} r_{2} r_{3} r_{4} \prod_{j=1}^{4} \Gamma_{p}\left(k+1-p r_{j}^{\prime}\right)} \\
& =\frac{\prod_{j=1}^{4}\left(r_{j}\right)_{k}}{k !^{4}} \prod_{j=1}^{4} \Gamma_{p}\left(r_{j}\right)\left(1+O\left(p^{2}\right)\right) \\
& =(-1)^{a_{1}+a_{2}} \frac{\prod_{j=1}^{4}\left(r_{j}\right)_{k}}{k !^{4}}\left(1+O\left(p^{2}\right)\right) .
\end{aligned}
$$

Furthermore,

$$
\begin{aligned}
- & \sum_{j=1}^{4} \sum_{i=1}^{a_{j}} \frac{1}{k+i-p r_{j}^{\prime}}+2 H_{k}-2 H_{p-1-k} \\
= & -\sum_{j=1}^{4}\left(G_{1}\left(k+1+a_{j}-p r_{j}^{\prime}\right)-G_{1}\left(k+1-p r_{j}^{\prime}\right)+\frac{\nu\left(a_{j},[-k-1]_{0}\right)}{p\left(1-r_{j}^{\prime}\right)}\right)+2 H_{k}-2 H_{p-1-k} \\
=- & \left(\sum_{j=1}^{4} G_{1}\left(k+1-r_{j}\right)-4 G_{1}(k+1)\right)-\sum_{j=1}^{4} \frac{\nu\left(k+1,\left[-a_{j}\right]_{0}\right)}{p\left(1-r_{j}^{\prime}\right)} \\
& +\sum_{j=1}^{4} G_{1}\left(k+1-p r_{j}^{\prime}\right)-4 G_{1}(1)-2 H_{k}-2 H_{p-1-k}
\end{aligned}
$$




$$
\begin{aligned}
& \equiv-\left(\sum_{j=1}^{4} G_{1}\left(k+1-r_{j}\right)-4 G_{1}(k+1)\right)-\sum_{j=1}^{4} \frac{\nu\left(k+1,\left[-a_{j}\right]_{0}\right)}{p\left(1-r_{j}^{\prime}\right)} \bmod p^{2} \\
& =-\left(J_{1}(k)+\sum_{j=1}^{4} \frac{\nu\left(k+1,\left[-a_{j}\right]_{0}\right)}{p\left(1-r_{j}^{\prime}\right)}\right),
\end{aligned}
$$

so that

$$
B_{k} \equiv(-1)^{a_{1}+a_{2}+1} \frac{\prod_{j=1}^{4}\left(r_{j}\right)_{k}}{k !^{4}} J_{1}(k) \bmod p^{2}
$$

for $0 \leq k \leq a_{1}$

$$
B_{k} \equiv(-1)^{a_{1}+a_{2}+1} \frac{\prod_{j=1}^{4}\left(r_{j}\right)_{k}}{k !^{4}}\left(J_{1}(k)+\frac{1}{p\left(1-r_{4}^{\prime}\right)}\right) \quad \bmod p^{2}
$$

for $a_{1}<k \leq a_{2}$;

$$
B_{k} \equiv(-1)^{a_{1}+a_{2}+1} \frac{\prod_{j=1}^{4}\left(r_{j}\right)_{k}}{k !^{4}}\left(J_{1}(k)+\frac{1}{p\left(1-r_{4}^{\prime}\right)}+\frac{1}{p\left(1-r_{3}^{\prime}\right)}\right) \quad \bmod p^{2}
$$

for $a_{2}<k \leq a_{3}$; and $B_{k} \equiv 0 \bmod p^{2}$ for $k>a_{3}$. Since $1-r_{4}^{\prime}=r_{1}^{\prime}$ and $1-r_{3}^{\prime}=r_{2}^{\prime}$, we obtain

$$
\begin{aligned}
(-1)^{a_{1}+a_{2}+1} \sum_{k=0}^{p-1} B_{k} \equiv \sum_{k=0}^{a_{3}} & \frac{\prod_{j=1}^{4}\left(r_{j}\right)_{k}}{k !^{4}} J_{1}(k) \\
& +\frac{1}{p r_{1}^{\prime}} \sum_{k=a_{1}+1}^{a_{3}} \frac{\prod_{j=1}^{4}\left(r_{j}\right)_{k}}{k !^{4}}+\frac{1}{p r_{2}^{\prime}} \sum_{k=a_{2}+1}^{a_{3}} \frac{\prod_{j=1}^{4}\left(r_{j}\right)_{k}}{k !^{4}} \bmod p^{2} .
\end{aligned}
$$

By comparing this with (26) and the formula defining $C_{1}$ we conclude that $C_{1} \equiv 0 \bmod p^{3}$.

We next show that $C_{2} \equiv 0 \bmod p^{3}$ using the different rational function

$$
\widetilde{R}(t)=\frac{\prod_{i=1}^{a_{1}}(t-i)}{\prod_{j=1}^{3} \prod_{i=0}^{a_{j}}(t+i)}=\sum_{k=0}^{a_{1}} \frac{\widetilde{A}_{k}}{(t+k)^{3}}+\sum_{k=0}^{a_{2}} \frac{\widetilde{B}_{k}}{(t+k)^{2}}+\sum_{k=0}^{a_{3}} \frac{\widetilde{D}_{k}}{t+k}
$$

and the related residue-sum identity

$$
\sum_{k=0}^{a_{3}} \widetilde{D}_{k}=\sum_{k=0}^{a_{3}} \operatorname{Res}_{t=-k} \widetilde{R}(t)=-\operatorname{Res}_{t=\infty} \widetilde{R}(t)=0
$$

for it. By construction, $\widetilde{R}(t)$ only has poles of order $j \in\{1,2,3\}$ at the points $t=-k$ with $a_{j-1}<k \leq a_{j}$ (recall the additional setting $a_{0}=-1$ ). With the argument used in the proof of Lemma 3 and reflection formula for the $p$-adic Gamma function we record

$$
\begin{aligned}
\left.\prod_{i=1}^{a_{1}}(t-i)\right|_{t=-k} & =(-1)^{k+1} \frac{\Gamma_{p}\left(k+1+a_{1}\right)}{k !}=\frac{\Gamma_{p}\left(k+1-r_{1}\right)}{\Gamma_{p}(k+1)}(1+O(p)) \\
& =\frac{\Gamma_{p}\left(k+r_{4}\right)}{\Gamma_{p}(k+1)}(1+O(p)) \quad \text { for } 0 \leq k \leq a_{3}
\end{aligned}
$$


and

$$
\begin{aligned}
\left.\frac{t+k}{\prod_{i=0}^{a_{j}}(t+i)}\right|_{t=-k} & =\frac{(-1)^{k}}{k !\left(a_{j}-k\right) !}=\frac{(-1)^{a_{j}-k}}{\Gamma_{p}(k+1) \Gamma_{p}\left(a_{j}-k+1\right)}=-\frac{\Gamma_{p}\left(k-a_{j}\right)}{\Gamma_{p}(k+1)} \\
& =-\frac{\Gamma_{p}\left(k+r_{j}\right)}{\Gamma_{p}(k+1)}(1+O(p)) \quad \text { for } 0 \leq k \leq a_{j} ; \\
\left.\frac{1}{\prod_{i=0}^{a_{j}}(t+i)}\right|_{t=-k} & =\frac{1}{(-k)_{a_{j}+1}}=\frac{(-1)^{a_{j}+1} \Gamma_{p}(-k)}{\Gamma_{p}\left(a_{j}-k+1\right)}=\frac{\Gamma_{p}\left(k-a_{j}\right)}{\Gamma_{p}(k+1)} \\
& =\frac{\Gamma_{p}\left(k+r_{j}\right)}{\Gamma_{p}(k+1)}(1+O(p)) \quad \text { for } a_{j}<k \leq a_{3},
\end{aligned}
$$

where $j=1,2,3$. Similar formulas but involving the functions $G_{1}$ and $G_{2}$ are valid for the $t$-derivatives of the left-hand sides and afterwards substitution $t=-k$, because all the terms in these formulas belong to $\mathbb{Z}_{p}^{\times}$: for any $s=0,1,2, \ldots$,

$$
\begin{aligned}
&\left.\frac{1}{s !} \frac{\mathrm{d}^{s}}{\mathrm{~d} t^{s}}\left(\prod_{i=1}^{a_{1}}(t-i)\right)\right|_{t=-k}\left.\equiv(-1)^{s} \frac{1}{s !} \frac{\mathrm{d}^{s}}{\mathrm{~d} t^{s}}\left(\frac{\Gamma_{p}\left(t+r_{4}\right)}{\Gamma_{p}(t+1)}\right)\right|_{t=k} \quad \bmod p \quad \text { for } 0 \leq k \leq a_{3} ; \\
&\left.\frac{1}{s !} \frac{\mathrm{d}^{s}}{\mathrm{~d} t^{s}}\left(\frac{t+k}{\prod_{i=0}^{a_{j}}(t+i)}\right)\right|_{t=-k} \equiv-\left.(-1)^{s} \frac{1}{s !} \frac{\mathrm{d}^{s}}{\mathrm{~d} t^{s}}\left(\frac{\Gamma_{p}\left(t+r_{j}\right)}{\Gamma_{p}(t+1)}\right)\right|_{t=k} \quad \bmod p \quad \text { for } 0 \leq k \leq a_{j} ; \\
&\left.\left.\frac{1}{s !} \frac{\mathrm{d}^{s}}{\mathrm{~d} t^{s}}\left(\frac{1}{\prod_{i=0}^{a_{j}}(t+i)}\right)\right|_{t=-k} \equiv(-1)^{s} \frac{1}{s !} \frac{\mathrm{d}^{s}}{\mathrm{~d} t^{s}}\left(\frac{\Gamma_{p}\left(t+r_{j}\right)}{\Gamma_{p}(t+1)}\right)\right|_{t=k} \bmod p \text { for } a_{j}<k \leq a_{3} .
\end{aligned}
$$

This computation implies that

$$
\begin{aligned}
\widetilde{D}_{k} & =\left.\frac{1}{(j-1) !} \frac{\mathrm{d}^{j-1}}{\mathrm{~d} t^{j-1}}\left(\widetilde{R}(t)(t+k)^{j}\right)\right|_{t=-k} \\
& \equiv-\left.\frac{1}{(j-1) !} \frac{\mathrm{d}^{j-1}}{\mathrm{~d} t^{j-1}}\left(\frac{\prod_{\ell=1}^{4} \Gamma_{p}\left(r_{\ell}+t\right)}{\Gamma_{p}(1+t)^{4}}\right)\right|_{t=k} \bmod p
\end{aligned}
$$

for $j=1,2,3$ and $a_{3-j}<k \leq a_{4-j}$; therefore,

$$
\begin{aligned}
-\sum_{k=0}^{a_{3}} \widetilde{D}_{k}=\left.\sum_{k=0}^{a_{1}} \frac{1}{2} \frac{\mathrm{d}^{2}}{\mathrm{~d} t^{2}}\left(\frac{\prod_{\ell=1}^{4} \Gamma_{p}\left(r_{\ell}+t\right)}{\Gamma_{p}(1+t)^{4}}\right)\right|_{t=k} \\
+\left.\sum_{k=a_{1}+1}^{a_{2}} \frac{\mathrm{d}}{\mathrm{d} t}\left(\frac{\prod_{\ell=1}^{4} \Gamma_{p}\left(r_{\ell}+t\right)}{\Gamma_{p}(1+t)^{4}}\right)\right|_{t=k} \\
+\left.\sum_{k=a_{2}+1}^{a_{3}} \frac{\prod_{\ell=1}^{4} \Gamma_{p}\left(r_{\ell}+t\right)}{\Gamma_{p}(1+t)^{4}}\right|_{t=k} \bmod p .
\end{aligned}
$$


Expanding the derivatives on the right-hand side of the resulting equality using both identities of Lemma 3 and (15) we arrive at

$$
\begin{aligned}
\left.\frac{\prod_{\ell=1}^{4} \Gamma_{p}\left(r_{\ell}+t\right)}{\Gamma_{p}(1+t)^{4}}\right|_{t=k} & =\frac{\prod_{\ell=1}^{4} \Gamma_{p}\left(r_{\ell}\right)}{k !^{4}} \frac{\prod_{\ell=1}^{4} \Gamma_{p}\left(r_{\ell}+k\right)}{\prod_{\ell=1}^{4} \Gamma_{p}\left(r_{\ell}\right)} \\
& =\frac{(-1)^{a_{1}+a_{2}}}{k !^{4}}\left(\frac{1}{p r_{1}^{\prime}}\right)^{\nu\left(k, a_{1}\right)}\left(\frac{1}{p r_{2}^{\prime}}\right)^{\nu\left(k, a_{2}\right)} \prod_{\ell=1}^{4}\left(r_{\ell}\right)_{k} .
\end{aligned}
$$

It follows that

$$
-p^{2} \sum_{k=0}^{a_{3}} \widetilde{D}_{k} \equiv(-1)^{a_{1}+a_{2}} C_{2} \bmod p^{3} .
$$

Thus, the residue sum formula (27) implies the desired congruence $C_{2} \equiv 0 \bmod p^{3}$ and completes the proof of Lemma 5 .

3.4. Companion congruences. We now record two companion congruences which will be employed in our second proof of Theorem 1, for all primes $p>5$ (not necessary ordinary). This is a bi-product of the derivation above, when the residue sum computation is performed for the rational functions $t R(t)$ and $t \widetilde{R}(t)$ in place of $R(t)$ and $\widetilde{R}(t)$, on using $\operatorname{Res}_{t=\infty} t R(t)=1$ (respectively, $\operatorname{Res}_{t=\infty} t \widetilde{R}(t)=0$ ) as well as (25). In these settings, the analysis in Section 3.3 reveals us with the following claim.

Lemma 6. We have

$$
\begin{aligned}
\widetilde{C}_{1}:= & p \sum_{k=0}^{a_{2}} \frac{\prod_{j=1}^{4}\left(r_{j}\right)_{k}}{k !^{4}}\left(J_{1}(k) k+1\right) \\
& \quad+\frac{1}{r_{1}^{\prime}} \sum_{k=a_{1}+1}^{a_{3}} \frac{\prod_{j=1}^{4}\left(r_{j}\right)_{k}}{k !^{4}} k+\frac{1}{r_{2}^{\prime}} \sum_{k=a_{2}+1}^{a_{3}} \frac{\prod_{j=1}^{4}\left(r_{j}\right)_{k}}{k !^{4}} k \\
\equiv & (-1)^{a_{1}+a_{2} p \bmod p^{3} ;} \\
\widetilde{C}_{2}:= & p^{2} \sum_{k=0}^{a_{1}} \frac{\prod_{j=1}^{4}\left(r_{j}\right)_{k}}{k !^{4}}\left(J_{2}(k) k^{2}+J_{1}(k) k\right) \\
& \quad+p \sum_{k=a_{1}+1}^{a_{2}} \frac{\prod_{j=1}^{4}\left(r_{j}\right)_{k}}{k !^{4}}\left(J_{1}(k) k+1\right) \frac{k}{r_{1}^{\prime}}+\frac{1}{r_{1}^{\prime} r_{2}^{\prime}} \sum_{k=a_{2}+1}^{a_{3}} \frac{\prod_{j=1}^{4}\left(r_{j}\right)_{k}}{k !^{4}} k^{2} \\
\equiv & -(-1)^{a_{1}+a_{2}} p^{2} \bmod p^{3} .
\end{aligned}
$$

We will use these congruences later in Section 6 for our different treatment of Theorem 1 , where $\widetilde{C}_{1}$ and $\widetilde{C}_{2}$ will play a role similar to that of $C_{1}$ and $C_{2}$ in the proof of Lemma 5 .

\section{Hypergeometric motives and the modularity OF RIGID CALABI-YAU THREEFOLDS}

It is now timely to formally introduce hypergeometric motives and their connection to both classical and finite hypergeometric functions, and to $L$-functions. The concept was proposed 
by Katz in [40] and developed into a fundamental object of study by Roberts, RodriguezVillegas and Watkins [65]. The main emphasis of such developments is on hypergeometric motives that admit lifts to $\mathbb{Q}$; all cases considered below fall into this category.

In the formulation of hypergeometric motives defined over $\mathbb{Q}$, the data consist of a parameter $\lambda \in \mathbb{Q}^{\times}$and two multi-sets

$$
\boldsymbol{\alpha}=\left\{\alpha_{1}, \ldots, \alpha_{m}\right\} \quad \text { and } \quad \boldsymbol{\beta}=\left\{\beta_{1}, \ldots, \beta_{m}\right\}
$$

from $\mathbb{Q}^{m}$, each closed under the Galois conjugation, that is, $\prod_{j=1}^{m}\left(x-e^{2 \pi i \alpha_{j}}\right) \in \mathbb{Z}[x]$ and $\prod_{j=1}^{m}\left(x-e^{2 \pi i \beta_{j}}\right) \in \mathbb{Z}[x]$. Recall that the closedness of $\boldsymbol{\alpha}$ under the Galois conjugation implies that $c \boldsymbol{\alpha} \equiv \boldsymbol{\alpha} \bmod \mathbb{Z}^{m}$ as multi-sets, for any non-zero $c \in \mathbb{Z}$ relatively prime to the common denominator of $\alpha_{1}, \ldots, \alpha_{m}$.

Additionally, assume that the hypergeometric data are primitive meaning that $\alpha_{j}-\beta_{\ell} \notin \mathbb{Z}$ for all $j$ and $\ell$. Under the latter constraint, there is an explicit way to numerically compute the $L$-function of generic degree $m$ attached to the hypergeometric data; the details are recorded in the notes [65] and the related Magma documentation on hypergeometric motives [77] by Watkins.

In the classical setting, when $\beta_{m}=1$ and none of $\beta_{j}$ is a non-negative integer, the corresponding (generalized) hypergeometric function is defined as

$$
\begin{aligned}
{ }_{m} F_{m-1}\left[\begin{array}{c}
\alpha_{1}, \alpha_{2}, \ldots, \alpha_{m} \\
\beta_{1}, \ldots, \beta_{m-1}
\end{array} ; \lambda\right] & =\sum_{k=0}^{\infty} \prod_{j=1}^{m} \frac{\left(\alpha_{j}\right)_{k}}{\left(\beta_{j}\right)_{k}} \lambda^{k} \\
& =\sum_{k \in \mathbb{Z}} \prod_{j=1}^{m} \frac{\Gamma\left(\alpha_{j}+k\right)}{\Gamma\left(\alpha_{j}\right)} \frac{\Gamma\left(1-\beta_{j}-k\right)}{\Gamma\left(1-\beta_{j}\right)}\left((-1)^{m} \lambda\right)^{k}
\end{aligned}
$$

where $(\alpha)_{k}=\Gamma(\alpha+k) / \Gamma(\alpha)$ as in the introduction.

We stress on the fact that the above conditions on hypergeometric data are invariant under shifts of any $\alpha_{j}$ or $\beta_{j}$ by integers; the invariance will be also featured by the finite-field analogue of the hypergeometric function. However, the hypergeometric function (28) itself is sensitive to such integer shifts - this is the subject of contiguous relations in the classical setting [5, Section 2.5]. To avoid unnecessary sophistication we will always assume that the multi-sets $\boldsymbol{\alpha}$ and $\boldsymbol{\beta}$ are normalized so that $0<\alpha_{j} \leq 1$ and $0<\beta_{j} \leq 1$ for $j=1, \ldots, m$. This is the case (3) we deal with in our applications.

4.1. Finite hypergeometric functions. The theory of finite hypergeometric functions was initiated by Greene [31] and Katz [40], with considerable developments over the recent years - see [10, 55, 67]. An interpretation of the theory in connection with Galois representations [40, 26] yields some fruitful results in computing zeta functions of algebraic varieties defined over finite fields.

We first fix some more notation. Let $\mathbb{F}_{q}$ denote the finite field of size $q$ and $\theta$ a fixed nontrivial additive character of $\mathbb{F}_{q}$. Let $\widehat{\mathbb{F}_{q}^{\times}}$be the set of all multiplicative characters of $\mathbb{F}_{q}$ with values in $\mathbb{C}_{p}^{\times}$, which is a cyclic group of size $q-1$, and $\omega$ a generator of $\widehat{\mathbb{F}_{q}^{\times}}$, the Teichmüller character, so that $\widehat{\mathbb{F}_{q}^{\times}}=\left\{\omega^{k}\right\}_{k=0}^{q-2}$. Denote by $\varepsilon$ the trivial multiplicative character. For any character $\chi \in \widehat{\mathbb{F}_{q}^{\times}}$including $\varepsilon$, we adapt the convention that $\chi(0)=0$. Define the Gauss sum 
of $\chi$ by

$$
g(\chi):=\sum_{x \in \mathbb{F}_{q}} \chi(x) \theta(x) .
$$

Under the hypothesis $(q-1) \alpha_{j},(q-1) \beta_{j} \in \mathbb{Z}$ for $j=1, \ldots, m$, Katz defines in [40, Section 8.2] the character sum

$$
H_{q}(\boldsymbol{\alpha}, \boldsymbol{\beta} ; \lambda)^{K}:=\frac{1}{q-1} \sum_{k=0}^{q-2} \omega^{-k}(\lambda) \prod_{j=1}^{m} g\left(\omega^{k+(q-1) \alpha_{j}}\right) g\left(\omega^{-k-(q-1) \beta_{j}}\right) \omega^{k+(q-1) \beta_{j}}(-1) .
$$

Following [10, Definition 1.1], we use the following modification. It corresponds to Katz's "canonical" version (independent of the choice of an additive character) in his later paper [42, Section 4]:

$$
H_{q}(\boldsymbol{\alpha}, \boldsymbol{\beta} ; \lambda):=\frac{1}{1-q} \sum_{k=0}^{q-2} \prod_{j=1}^{m}\left(\frac{g\left(\omega^{k+(q-1) \alpha_{j}}\right) g\left(\omega^{-k-(q-1) \beta_{j}}\right)}{g\left(\omega^{(q-1) \alpha_{j}}\right) g\left(\omega^{-(q-1) \beta_{j}}\right)}\right) \omega^{k}\left((-1)^{m} \lambda\right) .
$$

For $\boldsymbol{\alpha}=\left\{r_{1}, 1-r_{1}, r_{2}, 1-r_{2}\right\}, \beta=\{1,1,1,1\}$ and $\lambda=1$ as in our context, we have

$$
H_{q}(\boldsymbol{\alpha}, \beta ; 1)=-\omega^{(q-1)\left(r_{1}+r_{2}\right)}(-1) \cdot H_{q}(\boldsymbol{\alpha}, \beta ; 1)^{K} / q^{2} .
$$

Gauss sums are known to be finite-field analogues of the Gamma function values (see [26] for a dictionary between the classical and finite-field settings), therefore, the right-hand side of (30) reminisces (28). Next, we follow [10] to extend the definition to almost all finite fields $\mathbb{F}_{q}$, without the restriction on $q$ to satisfy $(q-1) \alpha_{j} \in \mathbb{Z}$ and $(q-1) \beta_{j} \in \mathbb{Z}$ for $j=1, \ldots, m$.

4.2. Finite hypergeometric sums. For a given integer $d \geq 1$, let

$$
\varphi(d)=\sum_{n \mid d} \mu\left(\frac{d}{n}\right) n
$$

denote the Euler number of $d$, where $\mu(\cdot)$ is the Möbius function. Write

$$
\frac{1}{(x-1)^{\varphi(d)}} \cdot \prod_{n \mid d}\left(x^{n}-1\right)^{\mu(d / n)}=\frac{\prod_{i=1}^{r}\left(x^{p_{i}}-1\right)}{\prod_{j=1}^{s}\left(x^{q_{j}}-1\right)}
$$

where $\left\{p_{i}\right\},\left\{q_{j}\right\}$ are disjoint multi-sets of positive integers, and define

$$
M_{d}:=\prod_{n \mid d}\left(n^{n}\right)^{\mu(d / n)} .
$$

Then it is not hard to verify (see [10]) that for $k \in \mathbb{Z}_{>0}$,

$$
\prod_{\substack{\ell=1 \\(\ell, d)=1}}^{d} \frac{(\ell / d)_{k}}{k !}=\frac{\left(p_{1} k\right) ! \cdots\left(p_{r} k\right) !}{\left(q_{1} k\right) ! \cdots\left(q_{s} k\right) !} M_{d}^{-k}=\frac{1}{k ! \varphi(d)} \prod_{n \mid d}\left((n k) ! n^{-n k}\right)^{\mu(d / n)}
$$

Now define a function $S_{d}(\chi)$ on characters $\chi \in \widehat{\mathbb{F}_{q}^{x}}$ by

$$
S_{d}(\chi):=g\left(\chi^{-1}\right)^{\varphi(d)} \cdot \prod_{n \mid d}\left(g\left(\chi^{n}\right) \chi\left(n^{-n}\right)\right)^{\mu(d / n)} .
$$


Noting that $k !=\Gamma(k+1)$ and $(n k) !=\Gamma(n k+1)$ we see that this is a finite-field analogue of the last term in (33).

Notice that each multi-set $\boldsymbol{\alpha}=\left\{\alpha_{1}, \ldots, \alpha_{m}\right\}$ closed under the Galois conjugation and satisfying $0<\alpha_{j} \leq 1$ for $j=1, \ldots, m$, can be partitioned in the form

$$
\boldsymbol{\alpha}=\bigcup_{i=1}^{t} \Sigma_{d_{i}}, \quad \text { where } \Sigma_{d}=\{\ell / d: 0<\ell \leq d,(\ell, d)=1\} \text { for } d \in\left\{d_{1}, \ldots, d_{t}\right\}
$$

with $\varphi\left(d_{1}\right)+\cdots+\varphi\left(d_{t}\right)=m$. The examples $\boldsymbol{\alpha}=\left\{r_{1}, 1-r_{1}, r_{2}, 1-r_{2}\right\}$ showing up in Theorem 1 all come from the multi-sets $\Sigma_{d}$ with $\varphi(d)=1,2$ or 4 ; the related data are collected in Table 2, Our next proposition is a version of a special case of Theorem $1.3 \mathrm{in}$ [10], in which we escape the use of reflection formula for the Gauss sums, so that denominators are kept in the intermediate character sums as they are.

Proposition 2. Assume that the hypergeometric data consist of $\lambda \in \mathbb{Q}^{\times}, \boldsymbol{\alpha}=\bigcup_{i=1}^{t} \Sigma_{d_{i}}$ and $\boldsymbol{\beta}=\{1, \ldots, 1\}$, and are primitive. Then for any finite field $\mathbb{F}_{q}$ with $q \equiv 1 \bmod d_{i}$ for all $i$,

$$
H_{q}(\boldsymbol{\alpha}, \boldsymbol{\beta} ; \lambda)=\frac{1}{1-q} \sum_{k=0}^{q-2} \prod_{i=1}^{t} S_{d_{i}}\left(\omega^{k}\right) \omega^{k}\left((-1)^{m} \lambda\right) .
$$

Proof. First, by [10] the finite hypergeometric function in (30) can be rewritten as

$$
H_{q}(\boldsymbol{\alpha}, \boldsymbol{\beta} ; \lambda)=\frac{1}{1-q} \sum_{k=0}^{q-2} \prod_{i=1}^{t} \mathrm{P}_{d_{i}}(k) \omega^{k}\left((-1)^{m} \lambda\right),
$$

where

$$
\mathrm{P}_{d}(k)=\prod_{\ell \in(\mathbb{Z} / d \mathbb{Z})^{\times}} \frac{g\left(\omega^{k+(q-1) \ell / d}\right) g\left(\omega^{-k}\right)}{g\left(\omega^{(q-1) \ell / d}\right) g(\varepsilon)}=g\left(\omega^{-k}\right)^{\varphi(d)} \prod_{\ell \in(\mathbb{Z} / d \mathbb{Z})^{\times}} \frac{g\left(\omega^{k+(q-1) \ell / d}\right)}{g\left(\omega^{(q-1) \ell / d}\right) g(\varepsilon)} .
$$

Following the proof of [10, Theorem 1.3], we can reformulate $\mathrm{P}_{d}(k)$ as

$$
\mathrm{P}_{d}(k)=g\left(\omega^{-k}\right)^{\varphi(d)} \prod_{n \mid d}\left(g\left(\omega^{n k}\right) \omega^{k}\left(n^{-n}\right)\right)^{\mu(d / n)}
$$

using the multiplication formula of Gauss sums (also known as the Hasse-Davenport relations [8, 10]). Notice that the function $\mathrm{P}_{d}(k)$ agrees with $S_{d}\left(\omega^{k}\right)$, hence the desired identity follows.

We remark that $H_{q}(\boldsymbol{\alpha}, \boldsymbol{\beta} ; \lambda)$ can be also given directly via Gauss sums (see [10, Definition 1.1]) and that the right-hand side of (36) is well defined for any finite field $\mathbb{F}_{q}$. Following [10], we use this more general definition of $H_{q}(\boldsymbol{\alpha}, \boldsymbol{\beta} ; \lambda)$ in further discussions.

4.3. Reduction to $p$-adic Gamma sums. The Gross-Koblitz formula [32] expresses Gauss sums $g(\chi)$ in terms of the $p$-adic Gamma function: for $0 \leq k \leq p-2$,

$$
g\left(\omega^{-k}\right)=-\pi_{p}^{k} \Gamma_{p}\left(\frac{k}{p-1}\right)
$$


TABle 2. Cyclotomic data

\begin{tabular}{|c|c|c|c|c|}
\hline$d$ & $\frac{\prod_{i=1}^{r}\left(x^{p_{i}}-1\right)}{\prod_{j=1}^{s}\left(x^{q_{j}}-1\right)}$ & $(r, s)$ & $\left\{p_{i}\right\},\left\{q_{j}\right\}$ & $M_{d}(\operatorname{see}(\underline{32}))$ \\
\hline 2 & $\frac{x^{2}-1}{(x-1)^{2}}$ & $(1,2)$ & $\begin{array}{c}p_{1}=2 \\
q_{1}=q_{2}=1\end{array}$ & $2^{2}$ \\
\hline 3 & $\frac{x^{3}-1}{(x-1)^{3}}$ & $(1,3)$ & $\begin{aligned} p_{1} & =3 \\
q_{1}=q_{2} & =q_{3}=1\end{aligned}$ & $3^{3}$ \\
\hline 4 & $\frac{x^{4}-1}{(x-1)^{2}\left(x^{2}-1\right)}$ & $(1,3)$ & $\begin{array}{c}p_{1}=4 \\
q_{1}=q_{2}=1, q_{3}=2\end{array}$ & $2^{6}$ \\
\hline 6 & $\frac{x^{6}-1}{(x-1)\left(x^{2}-1\right)\left(x^{3}-1\right)}$ & $(1,3)$ & $\begin{aligned} p_{1} & =6 \\
q_{1}=1, q_{2} & =2, q_{3}=3\end{aligned}$ & $2^{4} \cdot 3^{3}$ \\
\hline 5 & $\frac{x^{5}-1}{(x-1)^{5}}$ & $(1,5)$ & $\begin{array}{c}p_{1}=5 \\
q_{1}=\cdots=q_{5}=1\end{array}$ & $5^{5}$ \\
\hline 8 & $\frac{x^{8}-1}{(x-1)^{4}\left(x^{4}-1\right)}$ & $(1,5)$ & $\begin{aligned} p_{1} & =8 \\
q_{1}=\cdots & =q_{4}=1, \\
q_{5} & =4\end{aligned}$ & $2^{16}$ \\
\hline 10 & $\frac{x^{10}-1}{(x-1)^{3}\left(x^{2}-1\right)\left(x^{5}-1\right)}$ & $(1,5)$ & $\begin{array}{c}p_{1}=10 \\
q_{1}=q_{2}=q_{3}=1 \\
q_{4}=2, q_{5}=5\end{array}$ & $2^{8} \cdot 5^{5}$ \\
\hline 12 & $\frac{\left(x^{12}-1\right)\left(x^{2}-1\right)}{(x-1)^{4}\left(x^{4}-1\right)\left(x^{6}-1\right)}$ & $(2,6)$ & $\begin{array}{c}p_{1}=2, p_{2}=12 \\
q_{1}=\cdots=q_{4}=1 \\
q_{5}=4, q_{6}=6\end{array}$ & $2^{12} \cdot 3^{6}$ \\
\hline
\end{tabular}

where $\pi_{p}$ is a fixed root of $x^{p-1}+p=0$ in $\mathbb{C}_{p}$. For $p>2$ and an integer $n$ relatively prime with $p$, the product formula of the $p$-adic Gamma function [18, 57] reads

$$
\prod_{\ell=0}^{n-1} \Gamma_{p}\left(\frac{x+\ell}{n}\right)=\omega\left(n^{(1-x)(1-p)}\right) \Gamma_{p}(x) \prod_{\ell=1}^{n-1} \Gamma_{p}\left(\frac{\ell}{n}\right)
$$

where $x$ is of the form $m /(p-1)$ with $0 \leq m<p-1$.

Lemma 7. Given a prime $p>2$ and an integer $d>1$ such that $(d, p)=1$, the function $\nu(k, x)$ defined in (14) satisfies

$$
\sum_{\substack{\ell=1 \\ \ell, d)=1}}^{d} \nu\left(k, \frac{\ell}{d}(p-1)\right)=-\sum_{n \mid d} \mu\left(\frac{d}{n}\right)\left\lfloor\frac{-n k}{p-1}\right\rfloor .
$$

Proof. Consider

$$
f(x)=\nu(k, x(p-1))=-\left\lfloor x-\frac{k}{p-1}\right\rfloor
$$


and notice that

$$
\sum_{\ell=0}^{n-1} f\left(\frac{\ell}{n}\right)=-\left\lfloor-\frac{n k}{p-1}\right\rfloor
$$

since $\lfloor x\rfloor+\left\lfloor x+\frac{1}{n}\right\rfloor+\cdots+\left\lfloor x+\frac{n-1}{n}\right\rfloor=\lfloor n x\rfloor$ (see [63, Division 8, Problem 9]). It remains to combine this summation with the combinatorial identity

$$
\sum_{n \mid d} \mu\left(\frac{d}{n}\right) \sum_{\ell=0}^{n-1} f\left(\frac{\ell}{n}\right)=\sum_{n \mid d} \mu\left(\frac{d}{n}\right) \sum_{\ell=1}^{n-1} f\left(\frac{\ell}{n}\right)=\sum_{\substack{\ell=1 \\(\ell, d)=1}}^{d} f\left(\frac{\ell}{d}\right)
$$

valid for a generic function $f(x)$ defined on the interval $0 \leq x<1$ (see [63, Division 8, Problem 35]).

Lemma 8. Let an integer $d \geq 2$ be relatively prime with $p>2$. For the character sum defined in (34) and an integer $k, 0 \leq k \leq p-2$,

$$
S_{d}\left(\omega^{k}\right)=(-1)^{k \varphi(d)} \prod_{\substack{\ell=1 \\ \ell, d)=1}}^{d} \frac{\Gamma_{p}\left(\left\{\frac{\ell}{d}-\frac{k}{p-1}\right\}\right)}{\Gamma_{p}\left(\frac{\ell}{d}\right) \Gamma_{p}\left(1-\frac{k}{p-1}\right)}(-p)^{\nu(k, \ell(p-1) / d)},
$$

where $\nu(k, x)$ is as in (14).

Proof. Substitution of the Gross-Koblitz formula into (34) implies

$$
S_{d}\left(\omega^{k}\right)=(-1)^{\varphi(d)} \pi_{p}^{k \varphi(d)} \Gamma_{p}\left(\frac{k}{p-1}\right)^{\varphi(d)} \prod_{n \mid d}\left(-\pi_{p}^{(p-1)\left\{\frac{-n k}{p-1}\right\}} \Gamma_{p}\left(\left\{\frac{-n k}{p-1}\right\}\right) \omega^{k}\left(n^{-n}\right)\right)^{\mu(d / n)} .
$$

First notice that the exponent of $\pi_{p}$ on the right-hand side is equal to

$$
\begin{aligned}
k \varphi(d) & +(p-1) \sum_{n \mid d} \mu\left(\frac{d}{n}\right)\left\{\frac{-n k}{p-1}\right\} \\
& =k \varphi(d)+(p-1) \sum_{n \mid d} \mu\left(\frac{d}{n}\right) \cdot \frac{(-n k)}{p-1}-(p-1) \sum_{n \mid d} \mu\left(\frac{d}{n}\right)\left\lfloor\frac{-n k}{p-1}\right\rfloor=(p-1) \nu_{d}(k),
\end{aligned}
$$

where by Lemma 7

$$
\nu_{d}(k):=\sum_{\substack{\ell=1 \\(\ell, d)=1}}^{d} \nu\left(k, \frac{\ell}{d}(p-1)\right),
$$

reducing the character sum to

$$
S_{d}\left(\omega^{k}\right)=(-1)^{\varphi(d)}(-p)^{\nu_{d}(k)} \Gamma_{p}\left(\frac{k}{p-1}\right)^{\varphi(d)} \prod_{n \mid d}\left(\Gamma_{p}\left(\left\{\frac{-n k}{p-1}\right\}\right) \omega^{k}\left(n^{-n}\right)\right)^{\mu(d / n)} .
$$

For a real number $y$, the two sets

$$
\bigcup_{\ell=0}^{n-1} \frac{\{n y\}+\ell}{n}=\bigcup_{\ell=0}^{n-1}\left(y+\frac{\ell-\lfloor n y\rfloor}{n}\right) \text { and } \bigcup_{\ell=0}^{n-1}\left(y+\frac{\ell}{n}\right)
$$


coincide modulo $\mathbb{Z}$. Because the first set involves $n$ numbers equally distributed in the $n$ equal subintervals of $0 \leq x<1$, we conclude that

$$
\bigcup_{\ell=0}^{n-1} \frac{\{n y\}+\ell}{n}=\bigcup_{\ell=0}^{n-1}\left\{y+\frac{\ell}{n}\right\}
$$

Therefore, application of the product formula (37) with $x=\left\{\frac{-n k}{p-1}\right\}$ results in

$$
\begin{aligned}
\Gamma_{p}\left(\left\{\frac{-n k}{p-1}\right\}\right) \omega^{k}\left(n^{-n}\right) & =\omega^{p-1}\left(n^{1+\lfloor-n k /(p-1)\rfloor}\right) \frac{\prod_{\ell=0}^{n-1} \Gamma_{p}\left(\left\{-\frac{k}{p-1}+\frac{\ell}{n}\right\}\right)}{\prod_{\ell=1}^{n-1} \Gamma_{p}\left(\frac{\ell}{n}\right)} \\
& =\frac{\prod_{\ell=0}^{n-1} \Gamma_{p}\left(\left\{-\frac{k}{p-1}+\frac{\ell}{n}\right\}\right)}{\prod_{\ell=1}^{n-1} \Gamma_{p}\left(\frac{\ell}{n}\right)},
\end{aligned}
$$

as $\omega^{p-1}$ is the trivial character. Now, combining this formula with the combinatorial identity (38) and using

$$
\Gamma_{p}\left(\frac{k}{p-1}\right)^{\varphi(d)}=\frac{(-1)^{[k /(p-1)]_{0} \varphi(d)}}{\Gamma_{p}\left(1-\frac{k}{p-1}\right)^{\varphi(d)}}=(-1)^{[-k]_{0} \varphi(d)} \prod_{\substack{\ell=1 \\ \ell, d \\(\ell, d)=1}}^{d} \frac{1}{\Gamma_{p}\left(1-\frac{k}{p-1}\right)},
$$

we transform the right-hand side in (39) to the desired form.

With Lemma 8 at our disposal, we can give a different expression for a special case of the finite hypergeometric function, an expression that resembles the classical hypergeometric function (28).

Proposition 3. Assume that the hypergeometric data consist of $\lambda \in \mathbb{Q}^{\times}$, multi-sets $\boldsymbol{\alpha}=$ $\left\{\alpha_{1}, \ldots, \alpha_{m}\right\}$ and $\boldsymbol{\beta}=\{1, \ldots, 1\}$ closed under the Galois conjugation, and are primitive. Then for any finite field $\mathbb{F}_{p}$ with $p$ not dividing the least common denominator of $\alpha_{1}, \ldots, \alpha_{m}$,

$$
H_{p}(\boldsymbol{\alpha}, \boldsymbol{\beta} ; \lambda)=\frac{1}{\prod_{j=1}^{m} \Gamma_{p}\left(\alpha_{j}\right)} \frac{1}{1-p} \sum_{k=0}^{p-2} \frac{\prod_{j=1}^{m} \Gamma_{p}\left(\left\{\alpha_{j}-\frac{k}{p-1}\right\}\right)}{\Gamma_{p}\left(1-\frac{k}{p-1}\right)^{m}}(-1)^{k m}(-p)^{\nu_{\boldsymbol{\alpha}}(k)} \omega^{k}\left((-1)^{m} \lambda\right),
$$

where

$$
\nu_{\boldsymbol{\alpha}}(k):=\sum_{j=1}^{m} \nu\left(k, \alpha_{j}(p-1)\right) .
$$

Proof. Represent $\boldsymbol{\alpha}$ in the form (35) and apply Lemma 8 to get

$$
\prod_{i=1}^{t} S_{d_{i}}\left(\omega^{k}\right)=(-1)^{k m} \prod_{j=1}^{m} \frac{\Gamma_{p}\left(\left\{\alpha_{j}-\frac{k}{p-1}\right\}\right)}{\Gamma_{p}\left(\alpha_{j}\right) \Gamma_{p}\left(1-\frac{k}{p-1}\right)}(-p)^{\nu\left(k, \alpha_{j}(p-1)\right)} .
$$

The result then follows from Proposition 2 ,

Remark that the factor $\prod_{j=1}^{m} \Gamma_{p}\left(\alpha_{j}\right)$ in (40) is a quadratic character and the formula looks particularly friendly when the hypergeometric data (3) are as in Theorem 1:

$$
H_{p}(\boldsymbol{\alpha}, \boldsymbol{\beta} ; 1)=\frac{\chi_{\boldsymbol{\alpha}}(p)}{1-p} \sum_{k=0}^{p-2} \frac{\prod_{j=1}^{m} \Gamma_{p}\left(\left\{\alpha_{j}-\frac{k}{p-1}\right\}\right)}{\Gamma_{p}\left(1-\frac{k}{p-1}\right)^{m}}(-p)^{\nu_{\boldsymbol{\alpha}}(k)} .
$$




\section{Hypergeometric families of Calabi-Yau threefolds}

5.1. Algebraic models of hypergeometric Calabi-Yau threefolds. Each $\boldsymbol{\alpha}$ as stated in Theorem 1 corresponds to a one-parameter family of Calabi-Yau threefolds; see [16, Table 1] for their description and references. The construction was used in [50, 7] for hypersurfaces (and complete intersections) in projective spaces and later was extended from projective spaces to weighted projective spaces [60, 44]. Here a weighted projective space is denoted by $\mathbb{P}^{N}\left(w_{1}, \ldots, w_{N+1}\right)$, where $N$ is the dimension and $w_{i}$ denotes the weight of the corresponding variable $X_{i}$, while $X\left(n_{1}, \ldots, n_{r}\right)$ means the complete intersection of $r$ homogeneous polynomials of degree $n_{1}, \ldots, n_{r}$, respectively.

First, according to [60] there are four choices of $\boldsymbol{\alpha}$ that correspond to degree $n=\sum_{i=1}^{5} w_{i}$ homogeneous polynomials in $\mathbb{P}^{4}\left(w_{1}, \ldots, w_{5}\right)$ of the form

$$
V_{\boldsymbol{\alpha}}(\psi): \quad w_{1} X_{1}^{e_{1}}+w_{2} X_{2}^{e_{2}}+w_{3} X_{3}^{e_{3}}+w_{4} X_{4}^{e_{4}}+w_{5} X_{5}^{e_{5}}-n \psi X_{1} X_{2} X_{3} X_{4} X_{5}=0 .
$$

Compared to Table 1 of [60], the weights $w_{i}$ are added to the terms $X_{i}^{{ }^{e_{i}}}$ so that the $\psi$-values of the desired fibers are normalized to be equal to 1 . Each one is a one-parameter deformation of a Fermat type hypersurface $\sum_{i=1}^{5} w_{i} X_{i}^{e_{i}}=0$ with a large automorphism group

$$
G_{\boldsymbol{\alpha}}=\left\{\left(\zeta_{e_{1}}^{a_{1}}, \ldots, \zeta_{e_{5}}^{a_{5}}\right): \zeta_{e_{1}}^{a_{1}} \cdots \zeta_{e_{5}}^{a_{5}}=1\right\}
$$

For each $\psi \neq 0$, the fiber is a Calabi-Yau threefold. A classical paper on computing points of varieties like $V_{\boldsymbol{\alpha}}(\psi)$ over finite fields is [46] by Koblitz. In [70], Stienstra associated a one-dimensional commutative formal group over $\mathbb{Z}$ to each $V_{\boldsymbol{\alpha}}(\psi)$ with $\psi \in \mathbb{Z}$ in such a way that its formal logarithm

$$
\sum_{n \geq 1} \frac{A_{n}(\psi, \boldsymbol{\alpha})}{n} \tau^{n}
$$

(like in equation (5) of Section 2.1) satifsies, for almost all primes $p$,

$$
A_{p}(\psi, \boldsymbol{\alpha}) \equiv{ }_{4} F_{3}\left[\begin{array}{c}
r_{1}, 1-r_{1}, r_{2}, 1-r_{2} ; \lambda \\
1,1,1
\end{array}\right]_{p-1} \bmod p
$$

where $\lambda$ is the parameter of the mirror family $\mathcal{V}_{\boldsymbol{\alpha}}(\lambda)$. It is convenient to call $A_{p}(\psi, \boldsymbol{\alpha})$ a generalized Hasse invariant of the variety $V_{\boldsymbol{\alpha}}(\psi)$ at $p$. A good prime $p$ is ordinary for $V_{\boldsymbol{\alpha}}(\psi)$ when the value of the truncated hypergeometric series

$$
{ }_{4} F_{3}\left[\begin{array}{cc}
r_{1}, 1-r_{1}, r_{2}, 1-r_{2} \\
1, & 1,1
\end{array}\right]_{p-1}
$$

can be embedded into $\mathbb{Z}_{p}^{\times}$.

For other families, the defining equations are listed in Table 4. They are computed in the following way. First, the forth column is listed in [16] from which we know the number of equations, variables and the corresponding homogenous degree. Note that for the case $\left(\frac{1}{12}, \frac{5}{12}\right)$ we use $X(12,12)$ in $\mathbb{P}^{5}(1,1,4,6,6,6)$ instead of $X(2,12)$ in $\mathbb{P}^{5}(1,1,1,1,4,6)$. Then we look for each equations of one-parameter deformation of a Fermat type such that under the de-homogenization - the map like

$$
y_{i}=w_{i} X_{i}^{e_{i}} \text { for } i=1, \ldots, 5, \quad x_{1}=n \psi \prod_{i=1}^{5} X_{i}, \quad \lambda=\psi^{-n}
$$


TABLE 3. One-parameter families of hypersurfaces for $V_{\left\{r_{1}, r_{2}, 1-r_{1}, 1-r_{2}\right\}}(\psi)$

\begin{tabular}{|c|c|c||c|c|}
\hline$\left(d_{1}, \ldots, d_{t}\right)$ & $n$ & $\left(r_{1}, r_{2}\right)$ & $X(n) \in \mathbb{P}^{4}\left(w_{0}, \ldots, w_{4}\right)$ & Calabi-Yau threefold equation \\
\hline \hline$(5)$ & 5 & $\left(\frac{1}{5}, \frac{2}{5}\right)$ & $X(5) \subset \mathbb{P}^{4}(1,1,1,1,1)$ & $\sum_{j=1}^{5} X_{j}^{5}-5 \psi \prod_{j=1}^{5} X_{j}=0$ \\
\hline$(10)$ & 10 & $\left(\frac{1}{10}, \frac{3}{10}\right)$ & $X(10) \subset \mathbb{P}^{4}(1,1,1,2,5)$ & $\sum_{j=1}^{3} X_{j}^{10}+2 X_{4}^{5}+5 X_{5}^{2}-10 \psi \prod_{j=1}^{5} X_{j}=0$ \\
\hline$(8)$ & 8 & $\left(\frac{1}{8}, \frac{3}{8}\right)$ & $X(8) \subset \mathbb{P}^{4}(1,1,1,1,4)$ & $\sum_{j=1}^{4} X_{j}^{8}+4 X_{5}^{2}-8 \psi \prod_{j=1}^{5} X_{j}=0$ \\
\hline$(3,6)$ & 6 & $\left(\frac{1}{6}, \frac{1}{3}\right)$ & $X(6) \subset \mathbb{P}^{4}(1,1,1,1,2)$ & $\sum_{j=1}^{4} X_{j}^{6}+2 X_{5}^{3}-6 \psi \prod_{j=1}^{5} X_{j}=0$ \\
\hline
\end{tabular}

in the case of (42) - they are sent to the mirrors whose equations can be worked out using [10]; we outline this recipe below. Furthermore, we compute their generalized Hasse invariants as in [70] and verify that they agree with the truncated hypergeometric functions when reduced modulo $p$.

There are different ways, such as the Griffiths-Dwork method, to compute the PicardFuchs equation of such a family $V_{\boldsymbol{\alpha}}(\psi)$. A detailed computation was given in [60] by Morrison; the task can be alternatively done with the technology of GKZ hypergeometric functions [35]. For a discussion of computation using period integrals see the recent work [36] by Huang, Lian, Yau and Yu.

One way to obtain the mirror of $V_{\boldsymbol{\alpha}}(\psi)$ is via the orbifold $V_{\boldsymbol{\alpha}}(\psi) / G_{\boldsymbol{\alpha}}$ as described in Section 1 for the quintic case: one uses the map (43), which sends $V_{\boldsymbol{\alpha}}(\psi)$ to

$$
\hat{\mathcal{V}}_{\boldsymbol{\alpha}}(\lambda): \quad \sum_{i=1}^{5} y_{i}=x_{1}, \quad M_{n}^{-1} \lambda x_{1}^{n}=\prod_{i=1}^{5} y_{i}^{w_{i}}
$$

Such maps can be given for other hypergeometric cases and a general recipe, related to the discussion in [10, Section 6], is as follows. Using the partitioning (35) and Table 2 write

$$
\frac{\prod_{j=1}^{4}\left(x-e^{2 \pi i r_{j}}\right)}{(x-1)^{4}}=\frac{\left(x^{p_{1}}-1\right) \cdots\left(x^{p_{r}}-1\right)}{\left(x^{q_{1}}-1\right) \cdots\left(x^{q_{s}}-1\right)}
$$

so that $\left\{p_{i}\right\}$ and $\left\{q_{j}\right\}$ are disjoint multi-sets of positive integers. Set $\lambda=\psi^{-\left(p_{1}+\cdots+p_{r}\right)}$ and $\left(a_{1}, \ldots, a_{k}\right)=\left(p_{1}, \ldots, p_{r},-q_{1}, \ldots,-q_{s}\right)$, where $k=r+s$. For example, $\left(a_{i}\right)_{i=1, \ldots, 6}=$ $(6,-1,-1,-1,-1,-2)$ when $\boldsymbol{\alpha}=\left\{\frac{1}{3}, \frac{2}{3}, \frac{1}{6}, \frac{5}{6}\right\}$. Next fragmentate the index set $\{1,2, \ldots, k\}$ of $\left(a_{1}, a_{2}, \ldots, a_{k}\right)$ into a disjoint union of subsets $K_{1}, \ldots, K_{m}$ such that $\sum_{i \in K_{j}} a_{i}=0$ for each $j=1, \ldots, m$ and each part $\left(a_{i}\right)_{i \in K_{j}}$ cannot be fragmented likewise further; we call such fragmentations of the index set minimal. In the example above, the index set $\{1,2, \ldots, 6\}$ is already a minimal fragmentation, while the index set $\{1, \ldots, 8\}$ of $(6,4,-1,-3,-1,-1,-2,-2)$ (corresponding to $\boldsymbol{\alpha}=\left\{\frac{1}{4}, \frac{3}{4}, \frac{1}{6}, \frac{5}{6}\right\}$ ) can be fragmented into $K_{1}=\{1,3,4,7\}$ and $K_{2}=$ $\{2,5,6,8\}$ (this fragmentation is minimal but not unique - another minimal fragmentation can be found in the related row of Table 5 ). Then there is a natural morphism from the 
TABLE 4. Complete intersection of one-parameter families of hypersurfaces for $V_{\left\{r_{1}, r_{2}, 1-r_{1}, 1-r_{2}\right\}}(\psi)$

\begin{tabular}{|c|c|c|c|}
\hline$\left(d_{1}, \ldots, d_{t}\right)$ & $\left(r_{1}, r_{2}\right)$ & $X\left(n_{1}, \ldots, n_{r}\right)$ & Calabi-Yau threefold equations \\
\hline$(2,2,2,2)$ & $\left(\frac{1}{2}, \frac{1}{2}\right)$ & $X(2,2,2,2) \subset \mathbb{P}^{7}$ & $\begin{array}{c}X_{1}^{2}+X_{2}^{2}-2 \psi X_{3} X_{4}=0 \\
X_{3}^{2}+X_{4}^{2}-2 \psi X_{5} X_{6}=0 \\
X_{5}^{2}+X_{6}^{2}-2 \psi X_{7} X_{8}=0 \\
X_{7}^{2}+X_{8}^{2}-2 \psi X_{1} X_{2}=0 \\
\end{array}$ \\
\hline$(3,3)$ & $\left(\frac{1}{3}, \frac{1}{3}\right)$ & $X(3,3) \subset \mathbb{P}^{5}$ & $\begin{array}{l}X_{1}^{3}+X_{2}^{3}+X_{3}^{3}-3 \psi X_{4} X_{5} X_{6}=0 \\
X_{4}^{3}+X_{5}^{3}+X_{6}^{3}-3 \psi X_{1} X_{2} X_{3}=0\end{array}$ \\
\hline$(2,2,3)$ & $\left(\frac{1}{2}, \frac{1}{3}\right)$ & $X(2,2,3) \subset \mathbb{P}^{6}$ & $\begin{array}{c}X_{1}^{2}+X_{2}^{2}+X_{3}^{2}-3 \psi X_{4} X_{5}=0 \\
X_{4}^{3}+X_{5}^{3}-2 \psi X_{1} X_{6} X_{7}=0 \\
X_{6}^{2}+X_{7}^{2}-2 \psi X_{2} X_{3}=0\end{array}$ \\
\hline$(2,2,4)$ & $\left(\frac{1}{2}, \frac{1}{4}\right)$ & $X(2,4) \subset \mathbb{P}^{5}$ & $\begin{array}{c}X_{1}^{2}+X_{2}^{2}+X_{3}^{2}+X_{4}^{2}-4 \psi X_{5} X_{6}=0 \\
X_{5}^{4}+X_{6}^{4}-2 \psi X_{1} X_{2} X_{3} X_{4}=0 \\
\end{array}$ \\
\hline$(12)$ & $\left(\frac{1}{12}, \frac{5}{12}\right)$ & $X(12,12) \subset \mathbb{P}^{5}(1,1,4,6,6,6)$ & $\begin{array}{c}X_{1}^{12}+X_{2}^{12}-2 \psi X_{5} X_{6}=0 \\
X_{5}^{2}+X_{6}^{2}+4 X_{3}^{3}+6 X_{4}^{2}-12 \psi X_{1} X_{2} X_{3} X_{4}=0\end{array}$ \\
\hline$(4,4)$ & $\left(\frac{1}{4}, \frac{1}{4}\right)$ & $X(4,4) \subset \mathbb{P}^{5}(1,1,2,1,1,2)$ & $\begin{array}{r}X_{1}^{4}+X_{2}^{4}+2 X_{3}^{2}-4 \psi X_{4} X_{5} X_{6}=0 \\
X_{4}^{4}+X_{5}^{4}+2 X_{6}^{2}-4 \psi X_{1} X_{2} X_{3}=0 \\
\end{array}$ \\
\hline$(4,6)$ & $\left(\frac{1}{4}, \frac{1}{6}\right)$ & $X(4,6) \subset \mathbb{P}^{5}(1,1,2,1,2,3)$ & $\begin{array}{c}X_{1}^{4}+X_{2}^{4}+2 X_{3}^{2}+2 X_{5}^{2}-6 \psi X_{4} X_{6}=0 \\
X_{4}^{6}+3 X_{6}^{2}-4 \psi X_{1} X_{2} X_{3} X_{5}=0\end{array}$ \\
\hline$(3,4)$ & $\left(\frac{1}{3}, \frac{1}{4}\right)$ & $X(3,4) \subset \mathbb{P}^{5}(1,1,1,1,1,2)$ & $\begin{array}{c}X_{1}^{3}+X_{2}^{3}+X_{3}^{3}+X_{4}^{3}-4 \psi X_{5} X_{6}=0 \\
X_{5}^{4}+2 X_{6}^{2}-3 \psi X_{1} X_{2} X_{3} X_{4}=0\end{array}$ \\
\hline$(6,6)$ & $\left(\frac{1}{6}, \frac{1}{6}\right)$ & $X(6,6) \subset \mathbb{P}^{5}(1,2,3,1,2,3)$ & $\begin{array}{l}X_{1}^{6}+2 X_{2}^{3}+3 X_{3}^{2}-6 \psi X_{4} X_{5} X_{6}=0 \\
X_{4}^{6}+2 X_{5}^{3}+3 X_{6}^{2}-6 \psi X_{1} X_{2} X_{3}=0\end{array}$ \\
\hline$(2,2,6)$ & $\left(\frac{1}{2}, \frac{1}{6}\right)$ & $X(2,6) \subset \mathbb{P}^{5}(1,1,1,1,1,3)$ & $\begin{array}{c}3 X_{1}^{2}+X_{2}^{2}+X_{3}^{2}+X_{4}^{2}-6 \psi X_{1} X_{5}=0 \\
X_{5}^{6}+X_{6}^{2}-2 \psi X_{2} X_{3} X_{4} X_{6}=0\end{array}$ \\
\hline
\end{tabular}

corresponding homogeneous polynomial(s) in Tables 3 and 4 to

$$
W_{\boldsymbol{\alpha}}(\lambda): \quad \sum_{i \in K_{1}} x_{i}=\cdots=\sum_{i \in K_{m}} x_{i}=0, \quad \lambda M^{-1} x_{1}^{a_{1}} \cdots x_{k}^{a_{k}}=(-1)^{q_{1}+\cdots+q_{j}}
$$

where $M=M_{d_{1}} \cdots M_{d_{t}}$. Using the description of subsets $K_{1}, \cdots, K_{m}$ in Table 5 , the latter equation is the product of the $m$ equations

$$
\psi^{-p_{i}} M_{p_{i}}^{-1} \prod_{j \in K_{i}} x_{j}^{a_{j}}=\prod_{g_{j} \in K_{i}}(-1)^{q_{j}}, \quad \text { where } i=1, \ldots, m .
$$

With $\lambda=\psi^{-\left(p_{1}+\cdots+p_{r}\right)}$, the mirror family is then given by the following set of $2 m$ equations:

$$
\hat{\mathcal{V}}_{\boldsymbol{\alpha}}(\lambda): \quad \sum_{j \in K_{i}} x_{j}=0, \quad \psi^{-p_{i}} M_{p_{i}}^{-1} \prod_{j \in K_{i}} x_{j}^{a_{j}}=\prod_{g_{j} \in K_{i}}(-1)^{q_{j}}, \quad \text { where } i=1, \ldots, m .
$$

We record the information for each minor family in Table 5 , they are not unique - some instances differ from the descriptions in other sources, for example, in [17].

In the literature, there are many papers on counting the number of solutions of these algebraic equations of type (42) over finite fields; see, e.g., [24, 38, 39, 62, 76]. In particular, it is shown in [76] that $V_{\boldsymbol{\alpha}}(\psi)$ and its mirror $\hat{\mathcal{V}}_{\boldsymbol{\alpha}}(\lambda)$ share the same unit root. 
TABLE 5. Mirror families $\hat{\mathcal{V}}_{\left\{r_{1}, r_{2}, 1-r_{1}, 1-r_{2}\right\}}(\lambda)$

\begin{tabular}{|c|c|c|c|c|}
\hline$\left(r_{1}, r_{2}\right)$ & $M$ & $\left(a_{1}, \ldots, a_{k}\right)$ & fragmentation & $\operatorname{map} V_{\boldsymbol{\alpha}}(\psi) \rightarrow \mathcal{V}_{\boldsymbol{\alpha}}(\lambda)$ \\
\hline$\left(\frac{1}{5}, \frac{2}{5}\right)$ & $5^{5}$ & $(5,-1,-1,-1,-1,-1)$ & $\{1,2,3,4,5,6\}$ & $x_{1}=-5 \psi X_{1} \cdots X_{5}, x_{i+1}=X_{i}^{5}$ for $i=1,2,3,4,5, \lambda=\psi^{-5}$ \\
\hline$\left(\frac{1}{10}, \frac{3}{10}\right)$ & $2^{8} \cdot 5^{5}$ & $(10,-1,-1,-1,-2,-5)$ & $\{1,2,3,4,5,6\}$ & $\begin{array}{c}x_{1}=-10 \psi X_{1} \cdots X_{5}, x_{i+1}=X_{i}^{10} \text { for } i=1,2,3 \\
x_{5}=2 X_{4}^{5}, x_{6}=5 X_{5}^{2}, \lambda=\psi^{-10}\end{array}$ \\
\hline$\left(\frac{1}{8}, \frac{3}{8}\right)$ & $2^{16}$ & $(8,-1,-1,-1,-1,-4)$ & $\{1,2,3,4,5,6\}$ & $x_{1}=-8 \psi X_{1} \cdots X_{5}, x_{i+1}=X_{i}^{8}$ for $i=1,2,3,4, x_{6}=4 X_{5}^{2}, \lambda=\psi^{-8}$ \\
\hline$\left(\frac{1}{6}, \frac{1}{3}\right)$ & $2^{4} \cdot 3^{6}$ & $(6,-1,-1,-1,-1,-2)$ & $\{1,2,3,4,5,6\}$ & $x_{1}=-6 \psi X_{1} \cdots X_{5}, x_{i+1}=X_{i}^{6}$ for $i=1,2,3,4, x_{6}=2 X_{5}^{3}, \lambda=\psi^{-6}$ \\
\hline$\left(\frac{1}{2}, \frac{1}{2}\right)$ & $2^{8}$ & $(2,2,2,2,-1,-1,-1,-1,-1,-1,-1,-1)$ & $\begin{array}{c}\{1,5,6\},\{2,7,8\} \\
\{3,9,10\},\{4,11,12\}\end{array}$ & $\begin{array}{c}x_{1}=-2 \psi X_{3} X_{4}, x_{2}=-2 \psi X_{5} X_{6}, x_{3}=-2 \psi X_{7} X_{8} \\
x_{4}=-2 \psi X_{1} X_{2}, x_{4+i}=X_{i}^{2} \text { for } i=1, \ldots, 8, \lambda=\psi^{-8}\end{array}$ \\
\hline$\left(\frac{1}{3}, \frac{1}{3}\right)$ & $3^{6}$ & $(3,3,-1,-1,-1,-1,-1,-1)$ & $\begin{array}{l}\{1,3,4,5\} \\
\{2,6,7,8\} \\
\end{array}$ & $\begin{array}{c}x_{1}=-3 \psi X_{3} X_{4} X_{6}, x_{2}=-3 \psi X_{1} X_{2} X_{3} \\
x_{2+i}=X_{i}^{3} \text { for } i=1, \ldots, 6, \lambda=\psi^{-6}\end{array}$ \\
\hline$\left(\frac{1}{2}, \frac{1}{3}\right)$ & $2^{4} \cdot 3^{3}$ & $(3,2,2,-1,-1,-1,-1,-1,-1,-1)$ & $\begin{array}{c}\{1,4,5,6\} \\
\{2,7,8\},\{3,9,10\} \\
\end{array}$ & $\begin{array}{c}x_{1}=-3 \psi X_{4} X_{5}, x_{2}=-2 \psi X_{1} X_{6} X_{7}, x_{3}=-2 \psi X_{2} X_{3}, \\
x_{i+3}=X_{i}^{3} \text { for } i=1,2,3,4,5, x_{9}=X_{6}^{2}, x_{10}=X_{7}^{2}, \lambda=\psi^{-7}\end{array}$ \\
\hline$\left(\frac{1}{2}, \frac{1}{4}\right)$ & $2^{10}$ & $(4,2,-1,-1,-1,-1,-1,-1)$ & $\begin{array}{c}\{1,3,4,5,6\} \\
\{2,7,8\} \\
\end{array}$ & $\begin{array}{c}x_{1}=-4 \psi X_{5} X_{6}, x_{2}=-2 \psi X_{1} X_{2} X_{3} X_{4} \\
x_{i+2}=X_{i}^{2} \text { for } i=1,2,3,4, x_{7}=X_{5}^{4}, x_{8}=X_{6}^{4}, \lambda=\psi^{-6}\end{array}$ \\
\hline$\left(\frac{1}{12}, \frac{5}{12}\right)$ & $2^{12} \cdot 3^{6}$ & $(12,2,-1,-1,-4,-6,-1,-1)$ & $\begin{array}{c}\{1,3,4,5,6\} \\
\quad\{2,7,8\}\end{array}$ & $\begin{aligned} x_{1}= & -12 \psi X_{1} X_{2} X_{3} X_{4}, x_{2}=-2 \psi X_{5} X_{6}, x_{3}=X_{1}^{12}, x_{4}=X_{2}^{12}, \\
& x_{5}=X_{5}^{2}, x_{6}=X_{6}^{2}, x_{7}=4 X_{3}^{3}, x_{8}=6 X_{6}^{2}, \lambda=\psi^{-14}\end{aligned}$ \\
\hline$\left(\frac{1}{4}, \frac{1}{4}\right)$ & $2^{12}$ & $(4,4,-1,-1,-2,-1,-1,-2)$ & $\begin{array}{l}\{1,3,4,5\} \\
\{2,6,7,8\}\end{array}$ & $\begin{array}{c}x_{1}=-4 \psi X_{4} X_{5} X_{6}, x_{2}=-4 \psi X_{1} X_{2} X_{3} \\
x_{i+2}=X_{i}^{4} \text { for } i=1,2,4,5, x_{5}=2 X_{3}^{2}, x_{8}=2 X_{6}^{2}, \lambda=\psi^{-8}\end{array}$ \\
\hline$\left(\frac{1}{4}, \frac{1}{6}\right)$ & $2^{10} \cdot 3^{3}$ & $(6,4,-1,-3,-1,-1,-2,-2)$ & $\begin{array}{c}\{1,5,6,7,8\} \\
\quad\{2,3,4\}\end{array}$ & $\begin{array}{c}x_{1}=-6 \psi X_{4} X_{6}, x_{2}=-4 \psi X_{1} X_{2} X_{3} X_{5}, x_{3}=X_{4}^{6}, x_{4}=3 X_{6}^{2} \\
x_{5}=X_{1}^{4}, x_{6}=X_{2}^{4}, x_{7}=2 X_{3}^{2}, x_{8}=2 X_{5}^{2}, \lambda=\psi^{-10}\end{array}$ \\
\hline$\left(\frac{1}{3}, \frac{1}{4}\right)$ & $2^{6} \cdot 3^{3}$ & $(4,3,-1,-1,-1,-1,-1,-2)$ & $\begin{array}{l}\{1,3,4,5,6\} \\
\quad\{2,7,8\}\end{array}$ & $\begin{array}{c}x_{1}=-4 \psi X_{5} X_{6}, x_{2}=-3 \psi X_{1} X_{2} X_{3} X_{4} \\
x_{i+2}=X_{i}^{3} \text { for } i=1,2,3,4, x_{7}=x_{5}^{4}, x_{8}=2 X_{6}^{2}, \lambda=\psi^{-7}\end{array}$ \\
\hline$\left(\frac{1}{6}, \frac{1}{6}\right)$ & $2^{8} \cdot 3^{6}$ & $(6,6,-1,-2,-3,-1,-2,-3)$ & $\begin{array}{l}\{1,3,4,5\} \\
\{2,6,7,8\}\end{array}$ & $\begin{array}{c}x_{1}=-6 \psi X_{4} X_{5} X_{6}, x_{2}=-6 \psi X_{1} X_{2} X_{3}, x_{3}=X_{1}^{6}, x_{4}=2 X_{2}^{3} \\
x_{5}=3 X_{3}^{2}, x_{6}=X_{4}^{6}, x_{7}=2 X_{5}^{3}, x_{8}=3 X_{6}^{2}, \lambda=\psi^{-12}\end{array}$ \\
\hline$\left(\frac{1}{2}, \frac{1}{6}\right)$ & $2^{8} \cdot 3^{3}$ & $(6,2,-1,-1,-1,-1,-1,-3)$ & $\begin{array}{c}\{1,5,6,7,8\} \\
\{2,3,4\}\end{array}$ & $\begin{array}{c}x_{1}=-6 \psi X_{1} X_{5}, x_{2}=-2 \psi X_{2} X_{3} X_{4} X_{6} \\
x_{i+2}=X_{i}^{6} \text { for } i=1,2,3,4, x_{7}=X_{5}^{6}, x_{8}=3 X_{6}^{2}, \lambda=\psi^{-8}\end{array}$ \\
\hline
\end{tabular}


5.2. Modularity. For each of $\hat{\mathcal{V}}_{\boldsymbol{\alpha}}(\lambda)$, the smooth model of the fiber at $\lambda=1$ corresponds to a Calabi-Yau threefold $\hat{\mathcal{V}}_{\boldsymbol{\alpha}}(1)$ defined over $\mathbb{Q}$.

We first state a theorem due to Katz which is relevant to our discussion.

Theorem 4 (Katz). Let $r_{1}, r_{2}$ be as above, $N$ be the least positive common denominators of $r_{1}, r_{2}$, and $\boldsymbol{\alpha}=\left\{r_{1}, r_{2}, 1-r_{1}, 1-r_{2}\right\}, \boldsymbol{\beta}=\{1,1,1,1\}$. Assume that $\ell$ is a fixed prime dividing $N$ and $\lambda \in \mathbb{Z}[1 / N], \lambda \neq 0$. Take $K=\mathbb{Q}\left(\zeta_{N}\right)$. Then there is a continuous representation $\rho_{\boldsymbol{\alpha}, \ell, \lambda}$ of $G_{K}$, of degree 4 when $\lambda \neq 1$ and of degree 3 when $\lambda=1$, such that at each rational prime $p$ which splits completely in $\mathbb{Z}\left[\zeta_{N}\right]$,

$$
\operatorname{Tr} \rho_{\boldsymbol{\alpha}, \ell, \lambda}\left(\operatorname{Frob}_{p}\right)=H_{p}(\boldsymbol{\alpha}, \boldsymbol{\beta} ; \lambda) .
$$

Moreover, at $\lambda=1$, the representation $\rho_{\boldsymbol{\alpha}, \ell, 1}$ decomposes into a direct sum of 2-dimensional and 1-dimensional representations.

Proof. Let $\boldsymbol{\alpha}, \boldsymbol{\beta}$ be as above, and let $k=\mathbb{F}_{q}$ be a finite field such that $q \equiv 1 \bmod N, \psi$ a fixed nontrivial additive character of $k$. Let $k^{\text {sep }}$ denote a separable closure of $k$. Katz constructed in [40, Section 8.2] an $\ell$-adic sheaf $\mathcal{F}:=\mathcal{H}(!, \psi, \boldsymbol{\alpha}, \boldsymbol{\beta})$ of $\mathbb{G}_{m} / k$, which is lisse on $\mathbb{G}_{m}-\{1\}$ of rank 4 and is self-dual, as both $\boldsymbol{\alpha}, \boldsymbol{\beta}$ are defined over $\mathbb{Q}$.

At any geometric point $\bar{\lambda}$ of $\mathbb{G}_{m}\left(k^{\mathrm{sep}}\right)$ lying over $\lambda$, the stalk $\mathcal{F}_{\bar{\lambda}}$ of $\mathcal{F}$ gives rise to a generically degree 4 representation of

$$
\rho_{\lambda, \ell, \mathbb{F}_{q}}: \operatorname{Gal}\left(k^{\mathrm{sep}} / k\right) \rightarrow G L\left(\mathcal{F}_{\bar{\lambda}}\right),
$$

see [40, Section 7.3.7]. In particular, the trace of the geometric Frobenius element of $\operatorname{Gal}\left(k^{\text {sep }} / k\right)$ acting on the representation space $\mathcal{F}_{\bar{\lambda}}$ agrees with $-H_{q}(\boldsymbol{\alpha}, \boldsymbol{\beta} ; \lambda)^{K}$ which is recalled in (29), see [40, Section 8.2]. These representations are compatible when one considers finite extensions $E$ of $\mathbb{F}_{q}$. They also form a compatible family when $\lambda$ varies over $\mathbb{A}^{1} / \bar{k}$, where $\bar{k}$ is an algebraic closure of $k$. The function field of the algebraic variety $\mathbb{A}^{1} / \bar{k}$ is $\bar{k}(x)$ with an indeterminant $x$. Hence each stalk also admits the action of Gal $\left(\bar{k}(x)^{\operatorname{sep}} / \bar{k}(x)\right)$, which describes the coverings of $\mathbb{A}^{1}$ as a curve defined over $\bar{k}$. Each closed point $x$ of $\mathbb{A}^{1}$ can be viewed as a discrete valuation of $\bar{k}(x) / \bar{k}$. Fix a place $\tilde{x}$ of $\bar{k}(x)^{\text {sep }}$ lying over it and denote by $I(x)$ the inertia group of Gal $\left(\bar{k}(x)^{\mathrm{sep}} / \bar{k}(x)\right)$ at $\tilde{x}$. For each of the 14 cases, $\Lambda$ the product of the characters corresponding to upper and lower parameters is trivial (notation as (30); this means that $\Lambda=\prod_{j=1}^{4} \omega^{(q-1) \alpha_{j}} \prod_{j=1}^{4} \omega^{-(q-1) \beta_{j}}$ is the trivial character). From Theorem 8.4.2 in [40] and its proof we know that the inertia group $I(1)$ acts on $\mathcal{F}$ by a tame pseudoreflection (namely, its space of invariants has codimension 1, see [40, Section 7.6]). Consequently, the rank of $\mathcal{F}_{\overline{1}}$, as a vector space over $\overline{\mathbb{Q}}_{\ell}$, has a 1 dimension drop (see the proof of Theorem 8.4.11 (3) in [40]), meaning that the rank becomes 3. Moreover, from $\Lambda$ being trivial we know that the pseudoreflection at 1 is unipotent. By the Parity Recognition Theorem 8.8.2 [40, Theorem 8.8.2], the auto-duality pairing of $\mathcal{F}$ on the open subset of $\mathbb{G}_{m}-\{1\}$ has to be alternating. Therefore, a nontrivial subspace of $\mathcal{F}_{\overline{1}}$ also admits an alternating pairing, requiring the dimension of this subspace to be even, which can only be 2 in this case. Thus, $\rho_{1, \ell, \mathbb{F}_{q}}$ has a decomposition into one 1-dimensional and one 2-dimensional representations of $\operatorname{Gal}\left(\mathbb{F}_{q}^{\text {sep }} / \mathbb{F}_{q}\right)$. The subspace admitting alternating pairing has pure weight $4+4-1$ (see Section 7.3.7 in [40] for the definition of weight and Theorem 8.4.2 for the claim).

Later Katz modified $\mathcal{H}(!, \psi, \boldsymbol{\alpha}, \boldsymbol{\beta})$ by a "canonical" twist - see [42, Section 4] — to get $\mathcal{F}^{\text {can }}:=\mathcal{H}^{\text {can }}(\boldsymbol{\alpha} ; \boldsymbol{\beta})$. The trace of geometric Frobenius of $\mathbb{F}_{q}^{\text {sep }} / \mathbb{F}_{q}$ is given by $H_{q}(\boldsymbol{\alpha}, \boldsymbol{\beta} ; \lambda)$. 
Due to the canonical twisting, the 2-dimensional subspace has pure weight 3 (see (31) for the weight dropping by 4). The canonical twist removes the dependence on the additive character $\psi$ of $\mathbb{F}_{q}$ and hence allows the canonical sheaf $\mathcal{F}^{\text {can }}$ to be lifted from $\mathbb{G}_{m}$ over finite fields to over $\mathbb{Z}\left[\zeta_{N}\right]$; for the discussion on the inertia group $I(1), \bar{k}$ can be lifted to an algebraic closure of $K=\mathbb{Q}\left(\zeta_{N}\right)$, while $I(1)$ lifted to the inertia group of $\operatorname{Gal}\left(\bar{K}(x)^{\mathrm{sep}} / \bar{K}(x)\right)$ at 1 . Consequently, the stalk of $\mathcal{F}^{\text {can }}$ at $\lambda$ gives rise to a generically 4-dimensional representation $\rho_{\boldsymbol{\alpha}, \ell, \lambda}$ of $G_{K}$. At $\lambda=1$, it is 3-dimensional as the local monodromy at 1 is a unipotent pseudoreflection, which also forces the self-paring on a generic stalk to be alternating. Hence $\rho_{\boldsymbol{\alpha}, \ell, 1}$ decomposes as $\rho_{\boldsymbol{\alpha}, \ell, 1}^{(1)} \oplus \rho_{\boldsymbol{\alpha}, \ell, 1}^{(2)}$ of dimension 1 and 2 , respectively, where the representation space of the latter has pure weight 3 .

Proof of Theorem 2. Let $\lambda=1$. For the hypergeometric data (3) we perform the partitioning (35) and introduce the corresponding cyclotomic extension $K=\mathbb{Q}\left(e^{2 \pi i / d_{1}}, \ldots, e^{2 \pi i / d_{t}}\right)$. For each fixed prime $\ell$, and $\lambda \in \mathbb{Z}\left[1 /\left(\ell d_{1} \cdots d_{t}\right)\right]$, we know from Theorem 4 that there is an $\ell$-adic Galois representation $\rho_{\boldsymbol{\alpha}, \ell}:=\rho_{\boldsymbol{\alpha}, \ell, 1}$ of $G_{K}:=\operatorname{Gal}(\overline{\mathbb{Q}} / K)$ such that if $p \equiv 1\left(\bmod d_{j}\right)$ for $j=1, \ldots, t$, then

$$
H_{p}(\boldsymbol{\alpha}, \boldsymbol{\beta} ; 1)=\operatorname{Tr} \rho_{\boldsymbol{\alpha}, \ell}\left(\operatorname{Frob}_{p}\right) .
$$

Here $\mathrm{Frob}_{p}$ is the geometric Frobenius element at unramified prime $p$ (which splits completely in $K)$; in particular,

$$
H_{q}(\boldsymbol{\alpha}, \boldsymbol{\beta} ; 1)=\frac{1}{1-q} \sum_{\chi \in \widehat{\mathbb{F}}_{q}^{\times}} \prod_{j=1}^{t} S_{d_{j}}(\chi)
$$

as in (36) with $\omega^{k}=\chi$.

Since the hypergeometric motives are defined over $\mathbb{Q}$, the Galois representation $\rho_{\boldsymbol{\alpha}, \ell}$ can be extended to representations of the absolute Galois group $G_{\mathbb{Q}} 1$ These extensions are not unique, but their irreducible components are only differed by finite order characters of $G_{\mathbb{Q}}$ which fix $G_{K}$. In [10], Beukers, Cohen, and Mellit gave such an explicit extension, which is still denoted by $\rho_{\boldsymbol{\alpha}, \ell}$, via Gauss sum properties and a geometric realization using toric varieties compatible with Katz's formulation, which are $\hat{\mathcal{V}}_{\boldsymbol{\alpha}}(1)$ in our cases. The extended expression (46) applies to almost all $\mathbb{F}_{p}$ 's.

From the established modularity lifting theorems we know that the extension $\rho_{\boldsymbol{\alpha}, \ell}^{(2)}$ to $G_{\mathbb{Q}}$ is modular. For example, Theorem 2.1.4 of [6] states that given a prime $\ell$ and a 2dimensional absolutely irreducible representation of $G_{\mathbb{Q}}$ over $\overline{\mathbb{Q}}_{\ell}$ that is odd, unramified at almost all primes, and its restriction to the decomposition group $D_{\ell}$ at $\ell$ is crystalline with Hodge-Tate weight $\{0, r\}$, where $1 \leq r \leq \ell-2$ and $\ell+1 \nmid 2 r$, then $\rho$ is modular and corresponds to a weight $r+1$ Hecke eigenform. For our cases, we pick $\ell$ to be any prime larger than 5 and congruent to 1 modulo $N$. From Katz's construction, $\rho_{\boldsymbol{\alpha}, \ell}^{(2)}$ is unramified almost everywhere, odd and absolutely irreducible due to the alternating pairing which is Galois invariant, and its restriction to $D_{\ell}$ is crystalline with Hodge-Tate weight $\{0,3\}$. This means there exists a weight 4 normalized Hecke eigenform $f_{\boldsymbol{\alpha}}$ such that $\rho_{\boldsymbol{\alpha}, \ell}^{(2)}$ is isomorphic to the Deligne representation of $G_{\mathbb{Q}}$ associated with $f_{\boldsymbol{\alpha}}$. To determine the $f_{\boldsymbol{\alpha}}$ individually, we

\footnotetext{
${ }^{1}$ Assume that $H$ is a finite index subgroup of a group $G$. Up to semi-simplification, a representation $\rho$ of $H$ can be extended to a (non-unique) representation of $G$ if and only if $\rho \sim \rho^{g}$ (its conjugate by $g$ ) for all $g$ in $G / H$.
} 
compute the traces of $\rho_{\boldsymbol{\alpha}, \ell}^{(2)}\left(\operatorname{Frob}_{p}\right)$, using the hypergeometric motives routines implemented in Magma by Watkins via $H_{p}(\boldsymbol{\alpha}, \boldsymbol{\beta} ; 1)$ defined by (46) (see [77] and [65]). As each $\rho_{\boldsymbol{\alpha}, \ell}^{(2)}$ is unramifed outside the set $\{2,3,5, \ell\}$, we use a result of Serre (Theorem 2.2 in [23]), which asserts that the $p$-exponents of the level are bounded by 8 for $p=2$, by 5 for $p=3$, and by 2 for all other bad primes. This theorem reduces our search to a finite list. We used Magma to compute the first few coefficients of all Hecke eigenforms of level either dividing $2^{8} \cdot 3^{5}$ or $2^{8} \cdot 5^{2}$, they are available at the database. For the Sagemath program we used to identify the target modular forms, see the online Cocalc file. This allowed us to confirm the levels of $f_{\boldsymbol{\alpha}}$ listed in Table 1. Consequently, the extension of $\rho_{\boldsymbol{\alpha}, \ell}^{(1)}$ can be determined as well. For a closely related discussion, see a recent paper [49] by Li, Long and Tu.

For example, when $\left(r_{1}, r_{2}\right)=\left(\frac{1}{3}, \frac{1}{3}\right)$, the level of $f_{\boldsymbol{\alpha}}$ can only be $3,9,27,81$, or 243 . Computing in Magma all weight 4 Hecke eigenforms with integer coefficients of such levels and comparing them with the explicit values of $H_{p}(\boldsymbol{\alpha}, \boldsymbol{\beta} ; 1)$ at $p=5$ and 7 already identify $f_{\boldsymbol{\alpha}}$ as Entry 27.4.1.a in the database [47]. Other cases are verified in a similar fashion.

We end this section with the following remark. As mentioned by Rodriguez-Villegas in [66], the fiber at $\lambda=1$ for each of the fourteen hypergeometric families is a rigid Calabi-Yau threefold defined over $\mathbb{Q}$, and the modularity of rigid Calabi-Yau threefolds over $\mathbb{Q}$ is covered by the following theorem (see also [81] for more background).

Theorem 5 (Dieulefait [22], Gouvêa and Yui [30]). For each prime $\ell$, there is a weight 4 modular form $f_{\boldsymbol{\alpha}}$ with integer coefficient such that the $\ell$-adic Galois representation arising from the third étale cohomology group of $\hat{\mathcal{V}}_{\boldsymbol{\alpha}}(1)$ is isomorphic to the $\ell$-adic Deligne representation associated to $f_{\boldsymbol{\alpha}}$.

Therefore, it remains to provide an argument for verifying that $\overline{\hat{\mathcal{V}}_{\boldsymbol{\alpha}}(1)}$ is indeed a rigid Calabi-Yau threefold over $\mathbb{Q}$. Our argument mainly follows the idea explained in [78, Appendix] (see also [59] for the quintic case via point counting). Let $h^{i, j}$ denote the Hodge numbers of $\hat{\mathcal{V}}_{\alpha}(1)$. Denote by $N$ the least common denominator of $r_{1}$ and $r_{2}$. For a field $\mathbb{F}_{q}$ of characteristic $p>5$ such that $q \equiv 1(\bmod N)$, it follows from Theorem 6.1 in [10] that

$$
\# \overline{\hat{\mathcal{V}}_{\boldsymbol{\alpha}}(1)}\left(\mathbb{F}_{q}\right)=-H_{q}(\boldsymbol{\alpha}, \boldsymbol{\beta} ; 1)+F_{\boldsymbol{\alpha}}(q)
$$

where $F_{\boldsymbol{\alpha}}(q)$ is a polynomial in $q$ with integer coefficients. By Weil's (ex-)conjectures, the local zeta function of $\overline{\mathcal{V}}_{\boldsymbol{\alpha}}(1)$ over $\mathbb{F}_{q}$ assumes the form

$$
\exp \left(\sum_{r=1}^{\infty} \# \overline{\hat{\mathcal{V}}_{\boldsymbol{\alpha}}(1)} \frac{T^{r}}{r}\right)=\frac{f_{3}(T)}{(1-T)(1-q T)^{h^{1,1}}\left(1-q^{2} T\right)^{h^{2,1}}\left(1-q^{3} T\right)},
$$

where $f_{3}(T)$ is a polynomial of degree $2+2 h^{2,1}$ with all roots of absolute value $q^{3 / 2}$. By (47), there are only two roots of this absolute value, hence $h^{2,1}=0$ and we conclude that $\overline{\hat{\mathcal{V}}_{\boldsymbol{\alpha}}(1)}$ is rigid.

\section{Character-Sum Proof of Theorem 1}

Throughout the section a prime $p>5$ is fixed.

The finite hypergeometric function $H_{p}(\boldsymbol{\alpha}, \boldsymbol{\beta} ; 1)$, which is to be compared with the truncated ${ }_{4} F_{3}$ hypergeometric sum, is defined by means of character sums. In this section we use 
its non-character-sum representation from Proposition 3 and a methodology reminiscent to the one we had in Section 3. A similar argument was used in [43, 57] to cover two cases of Theorem 1.

As in Section [3, given one of the fourteen multi-sets $\boldsymbol{\alpha}=\left\{r_{1}, r_{2}, r_{3}, r_{4}\right\}$, we assume it labelled in such a way that the corresponding "derivative" multi-set $\left\{r_{1}^{\prime}, r_{2}^{\prime}, r_{3}^{\prime}, r_{4}^{\prime}\right\}$ is ordered: $r_{1}^{\prime} \leq r_{2}^{\prime} \leq r_{3}^{\prime} \leq r_{4}^{\prime}$. This implies that the integers $a_{j}:=\left[-r_{j}\right]_{0}=p r_{j}^{\prime}-r_{j}$ for $j=1,2,3,4$ are ordered accordingly, $a_{1} \leq a_{2} \leq a_{3} \leq a_{4}$, but also the pairing of the parameters:

$$
r_{1}+r_{4}=r_{2}+r_{3}=1, \quad r_{1}^{\prime}+r_{4}^{\prime}=r_{2}^{\prime}+r_{3}^{\prime}=1 \quad \text { and } \quad a_{1}+a_{4}=a_{2}+a_{3}=p-1
$$

Lemma 9. For $0 \leq k \leq p-2$, the following congruences hold true modulo $p^{3}$ :

$$
\prod_{j=1}^{4} \frac{\Gamma_{p}\left(\left\{r_{j}-\frac{k}{p-1}\right\}\right)(-p)^{\nu\left(k, r_{j}(p-1)\right)}}{\Gamma_{p}\left(r_{j}-\frac{k}{p-1}\right)} \equiv \begin{cases}1 & \text { if } 0 \leq k \leq a_{1}, \\ p\left(r_{1}^{\prime}-\frac{k}{p-1}\right) & \text { if } a_{1}+1 \leq k \leq a_{2}, \\ p^{2}\left(r_{1}^{\prime}-\frac{k}{p-1}\right)\left(r_{2}^{\prime}-\frac{k}{p-1}\right) & \text { if } a_{2}+1 \leq k \leq a_{3} \\ 0 & \text { if } k \geq a_{3}+1 .\end{cases}
$$

Proof. Observe that for each $j=1,2,3,4$, if $k \leq r_{j}(p-1)$ then

$$
\Gamma_{p}\left(\left\{r_{j}-\frac{k}{p-1}\right\}\right)(-p)^{\nu\left(k, r_{j}(p-1)\right)}=\Gamma_{p}\left(r_{j}-\frac{k}{p-1}\right) ;
$$

and if $r_{j}(p-1)<k<p-1$ then

$\Gamma_{p}\left(\left\{r_{j}-\frac{k}{p-1}\right\}\right)(-p)^{\nu\left(k, r_{j}(p-1)\right)}=-p \Gamma_{p}\left(1+r_{j}-\frac{k}{p-1}\right)=p\left(r_{j}-\frac{k}{p-1}\right)^{\star} \Gamma_{p}\left(r_{j}-\frac{k}{p-1}\right)$

where the factor in $(\cdot)^{\star}$ is omitted when divisible by $p$, that is, when $k=\left[-r_{j}\right]_{0}=a_{j}$. The latter can only happen when $a_{j}>r_{j}(p-1)$, equivalently, when $r_{j}<r_{j}^{\prime}$ (hence $r_{j}=r_{\ell}^{\prime}$ for some $\ell<j$ in view of the ordering). Thus, for a given $k, 0 \leq k \leq p-2$,

$$
\begin{aligned}
\prod_{j=1}^{4} \frac{\Gamma_{p}\left(\left\{r_{j}-\frac{k}{p-1}\right\}\right)(-p)^{\nu\left(k, r_{j}(p-1)\right)}}{\Gamma_{p}\left(r_{j}-\frac{k}{p-1}\right)}=\prod_{\substack{j=1 \\
r_{j}(p-1)<k}}^{4} p\left(r_{j}-\frac{k}{p-1}\right)^{\star} \\
=\prod_{j=1}^{4} p\left(r_{j}^{\prime}-\frac{k}{p-1}\right)^{\star}=\prod_{\substack{j=1 \\
r_{j}+r_{j}-r_{j}^{\prime}<k}}^{4} p\left(r_{j}^{\prime}-\frac{k}{p-1}\right)^{\star} \\
=\left\{\begin{array}{ll}
1 & \text { if } 0 \leq k \leq a_{1}, \\
p\left(r_{1}^{\prime}-\frac{k}{p-1}\right) & \text { if } a_{1}+1 \leq k<a_{2}, \\
p\left(r_{1}^{\prime}-\frac{k}{p-1}\right) & \text { if } k=a_{2} \text { and } r_{2} \geq r_{2}^{\prime}, \\
p^{2}\left(r_{2}^{\prime}-\frac{k}{p-1}\right) & \text { if } k=a_{2} \text { and } r_{2}<r_{2}^{\prime}, \\
p^{2}\left(r_{1}^{\prime}-\frac{k}{p-1}\right)\left(r_{2}^{\prime}-\frac{k}{p-1}\right) \\
p^{2}\left(r_{1}^{\prime}-\frac{k}{p-1}\right)\left(r_{2}^{\prime}-\frac{k}{p-1}\right) \\
p^{3}\left(r_{1}^{\prime}-\frac{k}{p-1}\right)\left(r_{2}^{\prime}-\frac{k}{p-1}\right)\left(r_{3}^{\prime}-\frac{k}{p-1}\right) /\left(r_{3}-\frac{k}{p-1}\right) & \text { if } a_{2}+1 \leq k<a_{3}, \\
0 \quad \bmod p^{3} & \text { if } k=a_{3} \text { and } r_{3} \geq r_{3}^{\prime},
\end{array},\right.
\end{aligned}
$$


In the case $k=a_{3}$ and $r_{3}<r_{3}^{\prime}$, we have either $r_{1}^{\prime}-\frac{k}{p-1}$ or $r_{2}^{\prime}-\frac{k}{p-1}$ divisible by $p$, so that

$$
p^{2}\left(r_{1}^{\prime}-\frac{k}{p-1}\right)\left(r_{2}^{\prime}-\frac{k}{p-1}\right) \equiv 0 \equiv p^{3} \frac{\left(r_{1}^{\prime}-\frac{k}{p-1}\right)\left(r_{2}^{\prime}-\frac{k}{p-1}\right)\left(r_{3}^{\prime}-\frac{k}{p-1}\right)}{r_{3}-\frac{k}{p-1}} \bmod p^{3} ;
$$

while in the case $k=a_{2}$ and $r_{2}<r_{2}^{\prime}$, hence $r_{2}=r_{1}^{\prime}$, we get

$$
\begin{aligned}
p^{2}\left(r_{2}^{\prime}-\frac{k}{p-1}\right) & =p^{2}\left(r_{2}^{\prime}-\frac{p r_{2}^{\prime}-r_{2}}{p-1}\right) \\
& =p\left(r_{2}-\frac{p r_{2}^{\prime}-r_{2}}{p-1}\right)=p\left(r_{1}^{\prime}-\frac{k}{p-1}\right)
\end{aligned}
$$

meaning that the expression obtained in this case agrees with the one for $k=a_{2}$ and $r_{2} \geq r_{2}^{\prime}$. This completes the proof of the lemma.

Using successively (22) and (18) we have

$$
\begin{aligned}
& \frac{\prod_{j=1}^{4} \Gamma_{p}\left(r_{j}-\frac{k}{p-1}\right)}{\Gamma_{p}\left(1-\frac{k}{p-1}\right)^{4} \prod_{j=1}^{4} \Gamma_{p}\left(r_{j}\right)}=\frac{\prod_{j=1}^{4} \Gamma_{p}\left(r_{j}+k+\frac{k p}{1-p}\right)}{\Gamma_{p}\left(1+k+\frac{k p}{1-p}\right)^{4} \prod_{j=1}^{4} \Gamma_{p}\left(r_{j}\right)} \\
& \quad \equiv \frac{\prod_{j=1}^{4} \Gamma_{p}\left(r_{j}+k\right)}{\Gamma_{p}(1+k)^{4} \prod_{j=1}^{4} \Gamma_{p}\left(r_{j}\right)}\left(1+J_{1}(k) \frac{k p}{1-p}+J_{2}(k) \frac{(k p)^{2}}{(1-p)^{2}}\right) \bmod p^{3} \\
& \quad=\frac{\prod_{j=1}^{4}\left(r_{j}\right)_{k}}{k !^{4} \prod_{j=1}^{4}\left(p r_{j}^{\prime}\right)^{\nu\left(k, a_{j}\right)}}\left(1+J_{1}(k) \frac{k p}{1-p}+J_{2}(k) \frac{(k p)^{2}}{(1-p)^{2}}\right),
\end{aligned}
$$

where $J_{1}(k)$ and $J_{2}(k)$ are defined in (23).

Combining the calculation and Lemma 9 we obtain the following: if $0 \leq k \leq a_{1}$ then

$$
\frac{\prod_{j=1}^{4} \Gamma_{p}\left(\left\{r_{j}-\frac{k}{p-1}\right\}\right)(-p)^{\nu\left(k, r_{j}(p-1)\right)}}{\Gamma_{p}\left(1-\frac{k}{p-1}\right)^{4} \prod_{j=1}^{4} \Gamma_{p}\left(r_{j}\right)} \equiv \frac{\prod_{j=1}^{4}\left(r_{j}\right)_{k}}{k !^{4}}\left(1+J_{1}(k) \frac{k p}{1-p}+J_{2}(k) \frac{(k p)^{2}}{(1-p)^{2}}\right) \quad \bmod p^{3} ;
$$

if $a_{1}+1 \leq k \leq a_{2}$ then

$$
\begin{aligned}
\frac{\prod_{j=1}^{4} \Gamma_{p}\left(\left\{r_{j}-\frac{k}{p-1}\right\}\right)(-p)^{\nu\left(k, r_{j}(p-1)\right)}}{\Gamma_{p}\left(1-\frac{k}{p-1}\right)^{4} \prod_{j=1}^{4} \Gamma_{p}\left(r_{j}\right)} & \equiv \frac{\prod_{j=1}^{4}\left(r_{j}\right)_{k}}{k !^{4}} \frac{r_{1}^{\prime}-\frac{k}{p-1}}{r_{1}^{\prime}}\left(1+J_{1}(k) \frac{k p}{1-p}\right) \bmod p^{3} \\
& \equiv \frac{\prod_{j=1}^{4}\left(r_{j}\right)_{k}}{k !^{4}}\left(1+\frac{k}{r_{1}^{\prime}}+\frac{k p}{r_{1}^{\prime}}\right)\left(1+J_{1}(k) k p\right) \bmod p^{3} ;
\end{aligned}
$$

if $a_{2}+1 \leq k \leq a_{3}$ then

$$
\begin{aligned}
\frac{\prod_{j=1}^{4} \Gamma_{p}\left(\left\{r_{j}-\frac{k}{p-1}\right\}\right)(-p)^{\nu\left(k, r_{j}(p-1)\right)}}{\Gamma_{p}\left(1-\frac{k}{p-1}\right)^{4} \prod_{j=1}^{4} \Gamma_{p}\left(r_{j}\right)} & \equiv \frac{\prod_{j=1}^{4}\left(r_{j}\right)_{k}}{k !^{4}} \frac{\left(r_{1}^{\prime}-\frac{k}{p-1}\right)\left(r_{2}^{\prime}-\frac{k}{p-1}\right)}{r_{1}^{\prime} r_{2}^{\prime}} \bmod p^{3} \\
& \equiv \frac{\prod_{j=1}^{4}\left(r_{j}\right)_{k}}{k !^{4}}\left(1+\frac{k}{r_{1}^{\prime}}\right)\left(1+\frac{k}{r_{2}^{\prime}}\right) \bmod p^{3} ;
\end{aligned}
$$


and if $k \geq a_{3}+1$ then

$$
\frac{\prod_{j=1}^{4} \Gamma_{p}\left(\left\{r_{j}-\frac{k}{p-1}\right\}\right)(-p)^{\nu\left(k, r_{j}(p-1)\right)}}{\Gamma_{p}\left(1-\frac{k}{p-1}\right)^{4} \prod_{j=1}^{4} \Gamma_{p}\left(r_{j}\right)} \equiv 0 \equiv \frac{\prod_{j=1}^{4}\left(r_{j}\right)_{k}}{k !^{4}} \quad \bmod p^{3} .
$$

Proposition 3 now implies

$$
H_{p}(\boldsymbol{\alpha}, \boldsymbol{\beta} ; 1) \equiv \sum_{k=0}^{p-1} \frac{\prod_{j=1}^{4}\left(r_{j}\right)_{k}}{k !^{4}}+\widetilde{C}_{1} \cdot \frac{1}{1-p}+\widetilde{C}_{2} \cdot\left(\frac{1}{1-p}\right)^{2} \bmod p^{3},
$$

where $\widetilde{C}_{1}$ and $\widetilde{C}_{2}$ are as in Lemma 6. From that lemma and also Lemma 4 we therefore conclude that

$$
H_{p}(\boldsymbol{\alpha}, \boldsymbol{\beta} ; 1) \equiv \sum_{k=0}^{p-1} \frac{\prod_{j=1}^{4}\left(r_{j}\right)_{k}}{k !^{4}}+\chi_{\boldsymbol{\alpha}}(p) \cdot p \bmod p^{3}
$$

Thus, Theorem 1 follows from comparison of this congruence with the equality (4). Combining this result with Theorem 2 one obtains for $p>5$,

$$
F_{1}(\boldsymbol{\alpha}) \equiv a_{p}\left(f_{\alpha}\right) \quad \bmod p^{3}
$$

\section{Conclusion}

For the convenience of readers, here we summarize our strategies used in this paper for establishing the supercongruences

Our first result is built on Dwork's work 25]; especially, on Dwork's dash operation which allows us to derive the key reduction formula (16). The formula is then used to separate the first $p$-adic digit $a$ of a non-negative integer $k=a+b p$ in ratios $(r)_{k} /(1)_{k}$ of rising factorials and re-express the ratios by means of $(r)_{a} /(1)_{a}$ and explicit additional terms. Combined with Proposition 1, this leaves the task of showing that two particular coefficients, $C_{1}$ and $C_{2}$, are both congruent to 0 modulo $p^{3}$. The latter is done by a residue sum calculation for certain rational functions and an execution of $p$-adic perturbation techniques.

In the character-sum approach to Theorem 1 we use our Theorem 2 and first express the hypergeometric sums $H_{p}(\boldsymbol{\alpha}, \boldsymbol{\beta} ; 1)$ in the $p$-adic Gamma function form (41), in which the arguments are fractional parts, via the Gross-Koblitz formula. Then Lemma 9 plays a role similar to that of formula (20) and simplifies the expression; the newer version of $H_{p}(\boldsymbol{\alpha}, \boldsymbol{\beta} ; 1)$ can be further expanded in powers of $p /(1-p)$ using local analyticity properties of the $p$-adic Gamma function. The remaining part is verifying that two coefficients in this expansion, $\tilde{C}_{1}$ and $\tilde{C}_{2}$, are both congruent to 0 modulo $p^{3}$, somewhat that has been already established earlier (in Lemma 6) as a companion to the congruences for $C_{1}$ and $C_{2}$.

Among all fourteen weight 4 modular forms corresponding to the rigid hypergeometric Calabi-Yau threefolds, only $f_{\left\{\frac{1}{4}, \frac{3}{4}, \frac{1}{3}, \frac{2}{3}\right\}}$ is a CM modular form:

$$
a_{p}\left(f_{\left\{\frac{1}{4}, \frac{3}{4}, \frac{1}{3}, \frac{2}{3}\right\}}\right)=J\left(\chi_{3}, \chi_{3}\right)^{3}+J\left(\chi_{3}^{2}, \chi_{3}^{2}\right)^{3},
$$

when $p \equiv 1 \bmod 3$ where $\chi_{3}$ stands for any cubic character of $\mathbb{F}_{p}$; so $p \equiv 1 \bmod 3$ is ordinary and the unit root is $\gamma_{p}=-\Gamma_{p}\left(\frac{1}{3}\right)^{9}$; for prime $p \equiv 2 \bmod 3$, the Fourier coefficient 
simply vanishes. Numerically we observe that the supercongruence for this case also reflects the additional CM structure: data suggest that for all primes $p \equiv 1 \bmod 3$,

$$
{ }_{4} F_{3}\left[\begin{array}{c}
\frac{1}{4}, \frac{3}{4}, \frac{1}{3}, \frac{2}{3} ; 1 \\
1,1,1
\end{array}\right]_{p-1} \equiv-\Gamma_{p}\left(\frac{1}{3}\right)^{9} \bmod p^{4},
$$

which is sharp. In comparison, the power $p^{3}$ is sharp in the statement of Theorem 1 for all other thirteen non-CM cases. Any proof of this observation may lead to new techniques in proving supercongruences of such kind.

One may also hope to extend horizons of another powerful method of "creative microscoping" [28, 29] used for proving hypergeometric supercongruences related to quadratic characters rather than general Dwork's unit roots. The ideas behind the method are based on suitable $(q$-)deformations to replace $p$-adic perturbations, and missing ingredients at present are connections with combinatorics ("counting") and geometry. Further development of this theme has potentials to also address the refined predictions from [64] of Roberts and Rodriguez-Villegas mentioned in Section 2.1.

Finally, we would mention that the techniques developed in this paper are applicable to numerous other supercongruences of "geometric" or "motivic" origin; in particular, to the hypergeometric patterns observed in [64].

\section{ACKNowledgements}

The authors would like to thank the Banff International Research Station and the MATRIX Institute at the University of Melbourne in Creswick for excellent opportunities for collaboration. The authors are grateful to the enlightening discussions and interests of many colleagues including Frits Beukers, Charles Doran, Jerome Hoffman, Siu-Hung Ng, Robert Osburn, Ravi Ramakrishna, David Roberts, Fernando Rodriguez-Villegas, Duco van Straten, Masha Vlasenko, Mark Watkins and Jie Zhou. The authors would like to further thank François Brunault and Michael Somos for supplying us with some explicit $\eta$-expressions in Table 1, as well as the anonymous referees for several constructive suggestions.

\section{Appendix A. The role of the Unit Root}

A.1. 1-CFGL. Closely related to Dwork's congruence (Proposition 1) is the theory of 1dimensional Commutative Formal Group Laws (1-CFGL) over a commutative ring $R$; see [72, Appendix] for a review of this topic. A 1-CFGL is a formal power series $G(u, v)=$ $u+v+$ higher degree terms $\in R[[u, v]]$ that satisfies commutativity and associativity. It is determined by its logarithm which takes the form

$$
\ell(\tau)=\sum_{n \geq 1} \frac{b_{n}}{n} \tau^{n}
$$

where $b_{n} \in R$ and $b_{1}=1$; namely, $G(u, v)=\ell^{-1}(\ell(u)+\ell(v))$. In [34], Honda showed that for an elliptic curve $E$ defined over $\mathbb{Z}$ with good reduction modulo $p$, the logarithm $\ell(\tau)$ of the associated formal group can be taken as the integral of the normalized invariant differential of $E$ expanded at infinity. When $b_{p} \neq \equiv 0(\bmod p)$, the $p$-adic limit

$$
\tilde{\gamma}_{p}=\lim _{s \rightarrow \infty} \frac{b_{p^{s}}}{b_{p^{s-1}}}
$$


is the reciprocal of the unique invertable in $\mathbb{Z}_{p}$ root of the denominator of the zeta function of $E$. In view of the modularity of $E$, this $\gamma_{p}$ is a root of $T^{2}-a_{p}\left(f_{E}\right) T+p=0$ with $\gamma_{p} \not \equiv 0(\bmod p)$, where $f_{E}$ is the weight 2 cuspidal Hecke eigenform associated with $E$. Generalizations of Honda's result include [80, Theorem 9] by the third author for formal Dirichlet series over an integral domain $R$ of characteristic 0 . For $V_{\boldsymbol{\alpha}}(\psi)$ listed in Table 4 , the following theorem of Stienstra will be useful to us.

Theorem 6 (Stienstra [70, Theorem 1]). Let $K$ be a noetherian ring which is flat over $\mathbb{Z}$. Let $F_{1}, \ldots, F_{r}$ be a regular sequence of homogenous polynomial in $K\left[T_{0}, \ldots, T_{N}\right]$ and let $X$ be the scheme of $\mathbb{P}_{K}^{N}$ defined by the ideal $\left(F_{1}, \ldots, F_{r}\right)$. Put $d_{i}=\operatorname{deg} F_{i}$ and $d=\sum_{i=1}^{r} d_{i}$. Assume that $X$ is flat over $K$ and $d_{i} \geq d-N \geq 1$ for all $i$. Let

$$
J=\left\{i=\left(i_{0}, \ldots, i_{N}\right) \in \mathbb{Z}^{N+1} \mid i_{0}, \ldots, i_{N} \geq 1, i_{0}+\ldots i_{N}=d\right\} .
$$

Then there is a formal group law for $H^{N-r}\left(X, \hat{G}_{m, \mathcal{O}_{X}}\right)$ over $K$ of dimension $n=\left(\begin{array}{c}d-1 \\ N\end{array}\right)$ whose logarithm $\ell(\tau)$ is the $n$-tuple $\left(\ell_{i}(\tau)\right)_{i \in J}$ of power series in $\tau=\left(\tau_{i}\right)_{i \in J}$ given by

$$
\ell_{i}(\tau)=\sum_{m \geq 1} \sum_{j \in J} m^{-1} \beta_{m, i, j} \tau_{j}^{m},
$$

where $\beta_{m, i, j}$ is the coefficient of $T_{0}^{m j_{0}-i_{0}} \cdots T_{N}^{m j_{N}-i_{N}}$ in $\left(F_{1} \cdots F_{r}\right)^{m-1}$.

Here, given a formal group $G$, a sheaf $\mathcal{J}$ of $K$-algebras on $X$ and $i \in \mathbb{Z}_{\geq 0}, H^{i}\left(X, G_{\mathcal{J}}\right)$ is a socalled Artin-Mazur functor from nilpotent $K$-algebras to abelian groups; see [70, Section 2]. For the above, $G=\hat{G}_{m}$, the multiplicative formal group law given by $\hat{G}_{m}(u, v)=u+v+u v$ for $u, v \in K$ and $\mathcal{O}_{X}$ is the structure sheaf of $X$.

A.2. 1-CFGL and rigid Calabi-Yau threefolds. We now explain how to use Theorem 6 to show that $\gamma_{p}=\gamma_{p}(\boldsymbol{\alpha})$ in Proposition 1 is related to the zeta function of a smooth model of $V(1)$. We will demonstrate this in the case of $\left(r_{1}, r_{2}\right)=\left(\frac{1}{2}, \frac{1}{2}\right)$. In this case, $V(\psi)$ is arising from the intersection of four hypersurfaces. Other cases are verified similarly. According to Table 4, the threefold $V(\psi)$ is the intersection of four homogenous equations $f_{1}=f_{2}=f_{3}=f_{4}=0$, where

$$
\begin{array}{ll}
f_{1}=X_{1}^{2}+X_{2}^{2}-2 \psi X_{3} X_{4}, & f_{2}=X_{3}^{2}+X_{4}^{2}-2 \psi X_{5} X_{6}, \\
f_{3}=X_{5}^{2}+X_{6}^{2}-2 \psi X_{7} X_{8}, & f_{4}=X_{7}^{2}+X_{8}^{2}-2 \psi X_{1} X_{2} .
\end{array}
$$

Take $Y(\psi)=f_{1} f_{2} f_{3} f_{4}$.

The sequence $f_{1}, f_{2}, f_{3}, f_{4}$ is regular (see [33, p. 184] for definition); we claim that, for any fixed $\psi \in \mathbb{Z}$, the scheme is flat over $K=\mathbb{Z}\left[(2 \psi)^{-1}\right]$, which is a PID.

Recall that over a PID, a module is flat if and only if it is torsion free. If $\mathfrak{f}$ in $R=$ $K\left[X_{1}, \ldots, X_{8}\right]$ is a torsion of $R / I$, then $r \cdot \mathfrak{f} \in I$ for some $r \in K$; that is, $\mathfrak{f}$ can be written as a linear combination of $f_{i}$ with coefficients in $\mathbb{Q}\left[X_{1}, \ldots, X_{8}\right]$. Explicitly, we can show that these coefficients are in $R$, hence $\mathfrak{f}$ must be trivial in $R / I$. We first use $f_{1}$ or $(2 \psi)^{-1} f_{4}$ to eliminate the presence of $X_{1}$ (and similarly of $X_{2}$ as they are interchangeable) from $\mathfrak{f}$ by replacing $X_{1}^{2}$ with $f_{1}-X_{2}^{2}+2 \psi X_{3} X_{4}$. After the elimination we write the reduced form of $\mathfrak{f}$ as $\mathfrak{f}_{1}+\mathfrak{f}_{2}$, where $\mathfrak{f}_{1} \in K\left[X_{3}, \ldots, X_{8}\right]$ and $\mathfrak{f}_{2} \in K\left[X_{1}, \ldots, X_{8}\right]\left[f_{1}, \ldots, f_{4}\right]$. Among them $\mathfrak{f}_{1}$ can be further written as a linear combination of $f_{2}, f_{3}$ with coefficients in $K\left[X_{3}, \ldots, X_{8}\right]$. This allows us to record $\mathfrak{f}$ as a linear combination of $f_{i}$ with coefficients in $K\left[X_{1}, \ldots, X_{8}\right]$. Thus, the scheme $X$ is indeed flat over the localized ring $K$. 
We next apply Theorem 6 with $K=\mathbb{Z}\left[(2 \psi)^{-1}\right]$. For the homogeneous polynomials $f_{i}$, degree $d_{i}=\operatorname{deg} f_{i}=2$ and $d=\sum_{i=1}^{4} d_{i}=8$. The dimension of the projective space is $N=8-1=7$, hence $d_{i}=2 \geq 1=d-N$ for each $i$. The set $J$ is $\left\{\left(i_{1}, \ldots, i_{8}\right) \in \mathbb{Z}^{8}: i_{i} \geq\right.$ $\left.1, i_{1}+\cdots+i_{8}=d\right\}=\{(1, \ldots, 1)\}$. By Theorem 6, $H^{3}\left(V(\psi), \hat{G}_{m, \mathcal{O}_{V(\psi)}}\right)$ is a 1-CFGL, and the $m$-th coefficient $b_{m}(\psi)$ of its logarithm is the coefficient of $\left(X_{1} \cdots X_{8}\right)^{m-1}$ of $\left(f_{1} \cdots f_{4}\right)^{m-1}$, namely,

$$
b_{m}(\psi)=2^{4 m-4} \sum_{k=0}^{m-1}\left(\begin{array}{c}
m-1 \\
2 k
\end{array}\right)^{4} \frac{\left(\frac{1}{2}\right)_{k}^{4}}{k !^{4}} \psi^{-8 k}=2^{4 m-4} \sum_{k \geq 0}^{m-1} \frac{\left(1-\frac{m}{2}\right)_{k}^{4}}{k !^{4}} \frac{\left(\frac{1}{2}-\frac{m}{2}\right)_{k}^{4}}{k !^{4}} \psi^{-8 k} .
$$

In particular, $b_{p}(1) \equiv F_{1}\left(\left\{\frac{1}{2}, \frac{1}{2}, \frac{1}{2}, \frac{1}{2}\right\}\right) \bmod p$ when $p>2$ is a prime. Assume from now on that $b_{p}(1) \not \equiv 0 \bmod p$, that is, $p$ is ordinary. Then by the theory of 1-CFGL [72, Theorem A.8(v)], there exists a unique $\tilde{\gamma}_{p} \in \mathbb{Z}_{p}^{\times}$such that

$$
\tilde{\gamma}_{p} \equiv \frac{b_{p^{s}}(1)}{b_{p^{s-1}}(1)} \quad \bmod p^{s} \quad \text { for all } s \geq 1
$$

Recall that by Proposition 1, for any ordinary odd prime $p$ there is a $p$-adic unit root $\gamma_{p}=\lim _{s \rightarrow \infty} F_{s} / F_{s-1}$. We use the following lemma to show that $\gamma_{p}=\tilde{\gamma}_{p}$.

Lemma 10. Let $p$ be an odd prime such that $b_{p}(1) \not \equiv 0 \bmod p$. Then for any fixed $m \geq 1$,

$$
\tilde{\gamma}_{p}=\lim _{s \rightarrow \infty} b_{p^{s}}(1) / b_{p^{s-1}}(1) \equiv \lim _{s \rightarrow \infty} F_{s} / F_{s-1} \equiv \gamma_{p} \quad \bmod p^{m}
$$

Proof. Let

$$
F(\lambda)=\sum_{k=0}^{\infty} \frac{\left(\frac{1}{2}\right)_{k}^{4}}{k !^{4}} \lambda^{k}
$$

By [25, Theorems 2 and 3], for any integer $s \geq 1$,

$$
F(x) \cdot{ }_{4} F_{3}\left[\begin{array}{c}
\frac{1}{2}, \frac{1}{2}, \frac{1}{2}, \frac{1}{2} ; x^{p} \\
1,1,1
\end{array}\right]_{p^{s-1}-1} \equiv F\left(x^{p}\right) \cdot{ }_{4} F_{3}\left[\begin{array}{c}
\frac{1}{2}, \frac{1}{2}, \frac{1}{2}, \frac{1}{2} \\
1,1,1
\end{array}\right]_{p^{s}-1} \bmod p^{s} \mathbb{Z}_{p}[[x]]
$$

where the truncations of the hypergeometric sum are defined as in (1). These congruences define the quotient $F(\lambda) / F\left(\lambda^{p}\right)$ as a uniform $p$-adic analytic function $f(\lambda)$, which assumes unit values on

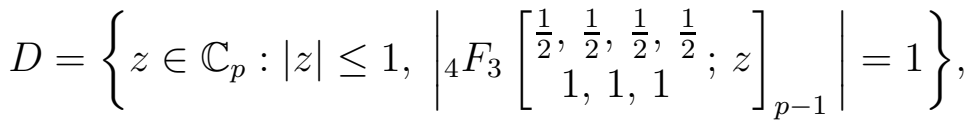

where $|\cdot|$ is the norm on $\mathbb{C}_{p}$. When $\lambda=1$ and $F_{1} \neq 0 \bmod p$,

$$
f(1)=\gamma_{p}=\lim _{s \rightarrow \infty} \frac{F_{s}(1)}{F_{s-1}(1)}
$$

by Proposition 1 .

Let $\lambda=\psi^{-8}$ and

$$
c_{p^{s}}(\lambda):=\sum_{k=0}^{\frac{p^{s}-1}{2}} \frac{\left(1-\frac{p^{s}}{2}\right)_{k}^{4}}{k !^{4}} \frac{\left(\frac{1}{2}-\frac{p^{s}}{2}\right)_{k}^{4}}{k !^{4}} \lambda^{k}
$$


so that $b_{p^{s}}(\psi)=2^{4\left(p^{s}-1\right)} c_{p^{s}}(\lambda)$ for $b_{p^{s}}(\psi)$ defined in (48). Note that $2^{4\left(p^{s}-p^{s-1}\right)} \equiv 1 \bmod p^{s}$. Under the ordinary assumption, meaning that $\lambda \in D$, by the property of 1-CFGL (see [72, Theorem A.8(v)] or [11, Part I, eq. (12)]), the $p$-adic limit

$$
\tilde{\gamma}(\lambda)=\lim _{s \rightarrow \infty} \frac{b_{p^{s}}(\psi)}{b_{p^{s-1}}\left(\psi^{p}\right)}
$$

exists and, for any $s \geq 1$,

$$
\frac{b_{p^{s}}(\psi)}{b_{p^{s-1}}\left(\psi^{p}\right)} \equiv \frac{c_{p^{s}}(\lambda)}{c_{p^{s-1}}\left(\lambda^{p}\right)} \bmod p^{s}
$$

Following the analysis of [11, Part I, Example 5.5], we conclude that the congruences

$$
\frac{\left(\frac{1-p^{s}}{2}\right)_{k}}{k !} \equiv \frac{\left(\frac{1}{2}\right)_{k}}{k !} \quad \bmod p^{s-\operatorname{ord}_{p}(k !)}, \quad \frac{\left(1-\frac{p^{s}}{2}\right)_{k}}{k !} \equiv 1 \quad \bmod p^{s-\operatorname{ord}_{p}(k !)},
$$

imply

$$
c_{p^{s}}(\lambda) \equiv F(\lambda) \bmod \left(\lambda^{s}, p^{\left\lfloor s \frac{p-2}{p-1}\right\rfloor}\right) \mathbb{Z}_{p}[[\lambda]]
$$

At the same time, we also have from 1-CFGL, for any fixed $m \geq 1$ and any $t, s \geq m$,

$$
\frac{c_{p^{t}}(\lambda)}{c_{p^{t-1}}\left(\lambda^{p}\right)} \equiv \frac{c_{p^{s}}(\lambda)}{c_{p^{s-1}}\left(\lambda^{p}\right)} \quad \bmod p^{m}
$$

Since both $s$ (the degree of $\lambda$ ) and $\left\lfloor s \frac{p-2}{p-1}\right\rfloor$ (the exponent of $p$ ) in the ideal $\left(\lambda^{s}, p^{\left\lfloor s \frac{p-2}{p-1}\right\rfloor}\right) \mathbb{Z}_{p}[[\lambda]]$ in (50) are increasing functions of $s$, by letting $s \rightarrow \infty$ in (50) we arrive at

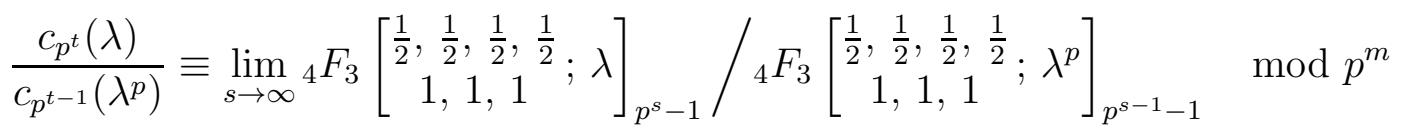

valid for any $m \geq 1$. Thus,

$$
\lim _{t \rightarrow \infty} \frac{c_{p^{t}}(\lambda)}{c_{p^{t-1}}\left(\lambda^{p}\right)}=f(\lambda)
$$

Choosing $\lambda=1$, the lemma follows.

In what follows we continue to assume $\psi=1$. Note that the singularities of algebraic threefolds can be resolved using essentially blowups to get smooth threefolds over a field of characteristic 0 (see [82]) or $p>5$ (see [1]). We denote by $X$ a smooth model of $V(1)$ over $\mathbb{F}_{p}$ of characteristic larger than 5. For the remaining of this section, we outline how Stienstra's results [71, §3.6] imply that $\gamma_{p}$ is related to the zeta function of $X$ (hence, of $V(1)$ ) via the crystalline cohomology groups $H_{\text {cris }}^{N}(X)$ (see [71] for definition and notation). A related discussion is available in the recent work [11, Appendix] of Beukers and Vlasenko on the Dwork $F$-crystal.

Recall from [71, Chapter 3] that the zeta function of $X$ over $\mathbb{F}_{p}$ is

$$
Z\left(X / \mathbb{F}_{p} ; T\right)=\prod_{N=0}^{6} P_{N}(T)^{(-1)^{N+1}}
$$

where $P_{N}(T)=\operatorname{det}\left(1-T F_{p} \mid H_{\text {cris }}^{N}(X) \otimes \mathbb{Q}\right)$ and $F_{p}$ is the Frobenius endomorphism on the de Rham-Witt complex on $X$ (see [71, §3.4]).

Proposition 4. In the notation above, $P_{3}\left(1 / \gamma_{p}\right)=0$. 
Proof. We give the steps of how the ingredients of Stienstra's work [70, 71] combine together. (i) Stienstra relates the CFGL groups of $Y(1)=0$ (defined by the hypersurface $f_{1} \cdots f_{4}=0$ ) and of $V(1)$ (the complete intersection of $f_{1}=0, \ldots, f_{4}=0$ ). Let $f: V(1) \rightarrow \mathbb{P}^{7}$ be the inclusion map, $\mathcal{O}_{V(1)}$ structure sheaf of $V(1)$ and $\mathcal{F}=f_{*} \mathcal{O}_{V(1)}$. For any non-empty proper subset $\rho \subset\{1,2,3,4\}$, denote $Y_{\rho}=\prod_{i \in \rho} f_{i}$; then $Y_{\rho}=0$ is a variety of $\mathbb{P}^{7}$ containing $V(1)$. Let $\mathcal{F}_{\rho}$ be the corresponding sheaf defined as for $\mathcal{F}$. In [70, Section 4], Stienstra shows the following two claims (see [70, Lemma 4.5 and (4.6.1)]):

$$
\begin{aligned}
& H^{i}\left(\mathbb{P}^{7}, \hat{G}_{m, \mathcal{F}_{\rho}}\right)=0 \quad \text { for } i=1, \ldots, 7, \\
& H^{7}\left(\mathbb{P}^{7}, \hat{G}_{m, \mathcal{F}}\right) \cong H^{3}\left(V(1), \hat{G}_{m, \mathcal{O}_{V(1)}}\right) .
\end{aligned}
$$

As the unit root $\gamma_{p}$ is computed from the formal logarithm of $H^{7}\left(\mathbb{P}^{7}, \hat{G}_{m, \mathcal{F}}\right)$, it can be computed from the formal logarithm of $H^{3}\left(V(1), \hat{G}_{m, \mathcal{O}_{V(1)}}\right)$.

(ii) To pass $\gamma_{p}$ from $H^{3}\left(V(1), \hat{G}_{m, \mathcal{O}_{V(1)}}\right)$ to $H^{3}\left(X, \hat{G}_{m, \mathcal{O}_{X}}\right)$, it is sufficient [71, $\left.\S 2.5\right]$ to show that the singularities of $V(1)$ are rational singularities (see [75, Definition 1]). (In our situation, the singularities of $V(1)$ are determined by the Jacobian matrix

$$
\left(\begin{array}{cccccccc}
2 X_{1} & 2 X_{2} & -2 X_{4} & -2 X_{3} & 0 & 0 & 0 & 0 \\
0 & 0 & 2 X_{3} & 2 X_{4} & -2 X_{6} & -2 X_{5} & 0 & 0 \\
0 & 0 & 0 & 0 & 2 X_{5} & 2 X_{6} & -2 X_{8} & -2 X_{7} \\
-2 X_{2} & -2 X_{1} & 0 & 0 & 0 & 0 & 2 X_{7} & 2 X_{8}
\end{array}\right)
$$

of rank less than four. The Jacobian gives rise to 96 isolated solutions in $\mathbb{C P}^{7}$ including, for example, $[1,1, \ldots, 1]$. The set of singularities can be verified using Magma package PrimaryComponents (SingularSubscheme(V)).) The singularities of $V(1)$ only consist of isolated ordinary double points, hence are rational singularities. They can be resolved individually by blowing up (see, for example, [33, Section 1.4] and [82]).

(iii) Finally, in [71, Section 3], Stienstra explains how to go from $H^{3}\left(X, \hat{G}_{m, \mathcal{O}_{X}}\right)$ to $H_{\text {cris }}^{3}(X)$. The particular relevant to us result is as follows.

Theorem 7 (Stienstra). Let $X$ be a smooth projective variety over $\mathbb{F}_{p}$ such that $H^{N}\left(X, \hat{G}_{m, X}\right)$ is a 1 -CFGL over $\mathbb{Z}_{p}$ with formal logarithm

$$
\sum_{m \geq 1} m^{-1} \beta_{m} \tau^{m}
$$

Take

$$
P_{N}(T)=\operatorname{det}\left(1-T F_{p} \mid H_{\text {cris }}^{N}(X) \otimes \mathbb{Q}\right)
$$

where $F_{p}$ is the Frobenius endomorphism on the de Rham-Witt complex on $X$. Then

$$
P_{N}(T)=1+a_{1} T+p a_{2} T+\cdots+p^{k-1} a_{k} T^{k} \quad \text { with } a_{1}, \ldots, a_{k} \in \mathbb{Z},
$$

where $k=\operatorname{deg} P_{N}(T)$ is the $N$-th Betti number of $X$, and for all integers $m, n \geq 1$,

$$
\beta_{m p^{n}}+a_{1} \beta_{m p^{n-1}}+p a_{2} \beta_{m p^{n-2}}+\cdots+p^{k-1} a_{k} \beta_{m p^{n-k}} \equiv 0 \bmod p^{n} .
$$


(iv) When $N=3$, we know that $H^{3}\left(X, \hat{G}_{m, \mathcal{O}_{X}}\right)$ is a 1-CFGL. If $\beta_{p} \not \equiv 0 \bmod p$, i.e. $p$ is ordinary, by [72, Theorem A.8(v)], for any $m, n \geq 1$,

$$
\frac{\beta_{m p^{n}}}{\beta_{m p^{n-1}}} \equiv \gamma_{p} \quad \bmod p^{n}
$$

where $\gamma_{p} \in \mathbb{Z}_{p}^{\times}$. Comparing with (53), when $m=n=1$, this implies that $\gamma_{p}=-a_{1} \bmod p$. When $m=1, n=2$, the congruence (53) translates after division by $\beta_{p}$ into

$$
\frac{\beta_{p^{2}}}{\beta_{p}}+a_{1}+p a_{2} \frac{\beta_{1}}{\beta_{p}} \equiv \gamma_{p}+a_{1}+\frac{p a_{2}}{\gamma_{p}} \equiv 0 \bmod p^{2},
$$

where (54) was used, resulting in

$$
\gamma_{p}^{2}+a_{1} \gamma_{p}+p a_{2} \equiv 0 \quad \bmod p^{2}
$$

Continuing this inductively we deduce, for any integer $n \geq 0$,

$$
\gamma_{p}^{k}+a_{1} \gamma_{p}^{k-1}+p a_{2} \gamma_{p}^{k-2}+\cdots+p^{k-1} a_{k} \equiv 0 \quad \bmod p^{k+n} .
$$

Thus, $P_{3}\left(1 / \gamma_{p}\right)=0$ as required.

The other thirteen cases are processed similarly.

A.3. The formal logarithms. In Table 6 we list the coefficients of the formal logarithms

$$
\sum_{m \geq 1} m^{-1} b_{m}(\psi, \boldsymbol{\alpha}) \tau^{m}
$$

for all fourteen cases $V_{\boldsymbol{\alpha}}(\psi)$.

\section{REFERENCES}

[1] S.S. Abhyankar, Resolution of singularities of embedded algebraic surfaces, Pure and Appl. Math. 24 (Academic Press, New York-London, 1966).

[2] A. Adolphson and S. Sperber, On unit root formulas for toric exponential sums, Algebra Number Theory 6 (2012), no. 3, 573-585.

[3] S. Ahlgren and K. Ono, A Gaussian hypergeometric series evaluation and Apéry number congruences, J. Reine Angew. Math. 518 (2000), 187-212.

[4] G. Almkvist, C. van Enckevort, D. van Straten, and W. Zudilin, Tables of Calabi-Yau equations, Preprint arXiv: math/0507430 [math.AG] (2005 \& 2010).

[5] G.E. Andrews, R. Askey, and R. Roy, Special functions, Encycl. Math. Appl. 71 (Cambridge University Press, Cambridge, 1999).

[6] A.O.L. Atkin, W.-C. Li, T. Liu, and L. Long, Galois representations with quaternion multiplication associated to noncongruence modular forms, Trans. Amer. Math. Soc. 365 (2013), 6217-6242.

[7] V. Batyrev and D. van Straten, Generalized hypergeometric functions and rational curves on Calabi-Yau complete intersections in toric varieties, Comm. Math. Phys. 168 (1995), 493-533.

[8] B.C. Berndt, R.J. Evans, and K.S. Williams, Gauss and Jacobi sums, Canad. Math. Soc. Ser. Monographs Adv. Texts, A Wiley-Interscience Publication (John Wiley \& Sons, Inc., New York, 1998).

[9] F. Beukers, Another congruence for the Apéry numbers, J. Number Theory 25 (1987), no. 2, 201-210.

[10] F. Beukers, H. Cohen, and A. Mellit, Finite hypergeometric functions, Pure Appl. Math. Q. 11 (2015), no. $4,559-589$.

[11] F. Beukers and M. Vlasenko, Dwork crystals I, Intern. Math. Res. Not. 2021 (2021), no. 12, 8807-8844; Dwork crystals II, Intern. Math. Res. Not. 2021 (2021), no. 6, 4427-4444.

[12] W. Bosma, J. Cannon, and C. Playoust, The Magma algebra system. I. The user language, J. Symbolic Comput. 24 (1997), 235-265. 
TABLE 6. Coefficients $b_{m}(\psi, \boldsymbol{\alpha})$ of the formal logarithms

\begin{tabular}{|c|c|c|}
\hline$\left(r_{1}, r_{2}\right)$ & $b_{m}(\psi, \boldsymbol{\alpha})$ & $\lambda$ \\
\hline$\left(\frac{1}{5}, \frac{2}{5}\right)$ & $(-5 \psi)^{m-1} \sum_{k \geq 0}\left(\begin{array}{c}m-1 \\
5 k\end{array}\right) \frac{\left(\frac{1}{5}\right)_{k}\left(\frac{2}{5}\right)_{k}\left(\frac{3}{5}\right)_{k}\left(\frac{4}{5}\right)_{k}}{k !^{4}}(-1)^{5 k} \lambda^{k}$ & $\psi^{-5}$ \\
\hline$\left(\frac{1}{10}, \frac{3}{10}\right)$ & $(-10 \psi)^{m-1} \sum_{k \geq 0}\left(\begin{array}{c}m-1 \\
10 k\end{array}\right) \frac{\left(\frac{1}{10}\right)_{k}\left(\frac{3}{10}\right)_{k}\left(\frac{7}{10}\right)_{k}\left(\frac{9}{10}\right)_{k}}{k !^{4}} \lambda^{k}$ & $\psi^{-10}$ \\
\hline$\left(\frac{1}{8}, \frac{3}{8}\right)$ & $(-8 \psi)^{m-1} \sum_{k \geq 0}\left(\begin{array}{c}m-1 \\
8 k\end{array}\right) \frac{\left(\frac{1}{8}\right)_{k}\left(\frac{3}{8}\right)_{k}\left(\frac{5}{8}\right)_{k}\left(\frac{7}{8}\right)_{k}}{k !^{4}} \lambda^{k}$ & $\psi^{-8}$ \\
\hline$\left(\frac{1}{6}, \frac{1}{3}\right)$ & $(-6 \psi)^{m-1} \sum_{k \geq 0}\left(\begin{array}{c}m-1 \\
6 k\end{array}\right) \frac{\left(\frac{1}{6}\right)_{k}\left(\frac{1}{3}\right)_{k}\left(\frac{2}{3}\right)_{k}\left(\frac{5}{6}\right)_{k}}{k !^{4}} \lambda^{k}$ & $\psi^{-6}$ \\
\hline$\left(\frac{1}{2}, \frac{1}{2}\right)$ & $(2 \psi)^{4 m-4} \sum_{k \geq 0}\left(\begin{array}{c}m-1 \\
2 k\end{array}\right)^{4} \frac{\left(\frac{1}{2}\right)_{k}^{4}}{k !^{4}} \lambda^{k}$ & $\psi^{-8}$ \\
\hline$\left(\frac{1}{3}, \frac{1}{3}\right)$ & $(3 \psi)^{2 m-2} \sum_{k \geq 0}\left(\begin{array}{c}m-1 \\
3 k\end{array}\right)^{2} \frac{\left(\frac{1}{3}\right)_{k}^{2}\left(\frac{2}{3}\right)_{k}^{2}}{k !^{4}} \lambda^{k}$ & $\psi^{-6}$ \\
\hline$\left(\frac{1}{2}, \frac{1}{3}\right)$ & $\left(-12 \psi^{3}\right)^{m-1} \sum_{k \geq 0}\left(\begin{array}{c}m-1 \\
2 k\end{array}\right)^{2}\left(\begin{array}{c}m-1 \\
3 k\end{array}\right) \frac{\left(\frac{1}{3}\right)_{k}\left(\frac{1}{2}\right)_{k}^{2}\left(\frac{2}{3}\right)_{k}}{k !^{4}}(-\lambda)^{k}$ & $\psi^{-7}$ \\
\hline$\left(\frac{1}{2}, \frac{1}{4}\right)$ & $\left(8 \psi^{2}\right)^{m-1} \sum_{k \geq 0}\left(\begin{array}{c}m-1 \\
2 k\end{array}\right)\left(\begin{array}{c}m-1 \\
4 k\end{array}\right) \frac{\left(\frac{1}{4}\right)_{k}\left(\frac{1}{2}\right)_{k}^{2}\left(\frac{3}{4}\right)_{k}}{k !^{4}} \lambda^{k}$ & $\psi^{-6}$ \\
\hline$\left(\frac{1}{12}, \frac{5}{12}\right)$ & $\left(24 \psi^{2}\right)^{m-1} \sum_{k \geq 0}\left(\begin{array}{c}m-1 \\
2 k\end{array}\right)\left(\begin{array}{c}m-1 \\
12 k\end{array}\right) \frac{\left(\frac{1}{12}\right)_{k}\left(\frac{5}{12}\right)_{k}\left(\frac{7}{12}\right)_{k}\left(\frac{11}{12}\right)_{k}}{k !^{4}} \lambda^{k}$ & $\psi^{-14}$ \\
\hline$\left(\frac{1}{4}, \frac{1}{4}\right)$ & $(4 \psi)^{2 m-2} \sum_{k \geq 0}\left(\begin{array}{c}m-1 \\
4 k\end{array}\right)^{2} \frac{\left(\frac{1}{4}\right)_{k}^{2}\left(\frac{3}{4}\right)_{k}^{2}}{k !^{4}} \lambda^{k}$ & $\psi^{-8}$ \\
\hline$\left(\frac{1}{4}, \frac{1}{6}\right)$ & $\left(24 \psi^{2}\right)^{m-1} \sum_{k \geq 0}\left(\begin{array}{c}m-1 \\
4 k\end{array}\right)\left(\begin{array}{c}m-1 \\
6 k\end{array}\right) \frac{\left(\frac{1}{6}\right)_{k}\left(\frac{1}{4}\right)_{k}\left(\frac{3}{4}\right)_{k}\left(\frac{5}{6}\right)_{k}}{k !^{4}} \lambda^{k}$ & $\psi^{-10}$ \\
\hline$\left(\frac{1}{3}, \frac{1}{4}\right)$ & $\left(12 \psi^{2}\right)^{m-1} \sum_{k \geq 0}\left(\begin{array}{c}m-1 \\
3 k\end{array}\right)\left(\begin{array}{c}m-1 \\
4 k\end{array}\right) \frac{\left(\frac{1}{4}\right)_{k}\left(\frac{1}{3}\right)_{k}\left(\frac{2}{3}\right)_{k}\left(\frac{3}{4}\right)_{k}}{k !^{4}}(-\lambda)^{k}$ & $\psi^{-7}$ \\
\hline$\left(\frac{1}{6}, \frac{1}{6}\right)$ & $(6 \psi)^{2 m-2} \sum_{k \geq 0}\left(\begin{array}{c}m-1 \\
6 k\end{array}\right)^{2} \frac{\left(\frac{1}{6}\right)_{k}^{2}\left(\frac{5}{6}\right)_{k}^{2}}{k !^{4}} \lambda^{k}$ & $\psi^{-12}$ \\
\hline$\left(\frac{1}{2}, \frac{1}{6}\right)$ & $\left(12 \psi^{2}\right)^{m-1} \sum_{k>0}\left(\begin{array}{c}m-1 \\
2 k\end{array}\right)\left(\begin{array}{c}m-1 \\
6 k\end{array}\right) \frac{\left(\frac{1}{6}\right)_{k}\left(\frac{1}{2}\right)_{k}^{2}\left(\frac{5}{6}\right)_{k}}{k !^{4}} \lambda^{k}$ & $\psi^{-8}$ \\
\hline
\end{tabular}

[13] P. Candelas, X. de la Ossa, P.S. Green, and L. Parkes, A pair of Calabi-Yau manifolds as an exactly soluble superconformal theory, Nuclear Phys. B 359 (1991), no. 1, 21-74. 
[14] P. Candelas, X. de la Ossa, and F. Rodriguez-Villegas, Calabi-Yau manifolds over finite fields I, Preprint arXiv: hep-th/0012233 (2015).

[15] H.H. Chan, L. Long, and W. Zudilin, A supercongruence motivated by the Legendre family of elliptic curves, Math. Notes 88 (2010), no. 4, 599-602.

[16] Y.-H. Chen, Y. Yang, and N. Yui, Monodromy of Picard-Fuchs differential equations for Calabi-Yau threefolds. With an appendix by C. Erdenberger, J. Reine Angew. Math. 616 (2008), 167-203.

[17] A. Clingher, C.F. Doran, J. Lewis, A.Y. Novoseltsev, and A. Thompson, The 14th case VHS via K3 fibrations, in: Recent advances in Hodge theory: Period domains, algebraic cycles and arithmetic, London Math. Soc. Lecture Note Ser. 427 (Cambridge University Press, Cambridge, 2016), 165-227.

[18] H. Cohen, Number theory. Vol. II. Analytic and modern tools, Graduate Texts in Math. 240 (Springer, New York, 2007).

[19] D.A. Cox, Mirror symmetry and polar duality of polytopes, Symmetry 7 (2015), no. 3, 1633-1645.

[20] A. Deines, J. Fuselier, L. Long, H. Swisher, and F.-T. Tu, Hypergeometric series, truncated hypergeometric series, and Gaussian hypergeometric functions, in: Directions in number theory, Assoc. Women Math. Ser. 3 (Springer, New York, 2016), 125-159.

[21] L. Dembélé, A. Panchishkin, J. Voight, and W. Zudilin, Special hypergeometric motives and their L-Functions: Asai recognition, Experiment. Math. (to appear), 13 pp.; doi: 10.1080/10586458.2020.1737990.

[22] L. Dieulefait, On the modularity of rigid Calabi-Yau threefolds: epilogue, J. Math. Sci. 171 (2010), no. 6, 725-727.

[23] L. Dieulefait, Computing the level of a modular rigid Calabi-Yau threefold, Experiment. Math. 13 (2004), no. 2, 165-169.

[24] C.F. Doran, T.L. Kelly, A. Salerno, S. Sperber, J. Voight, and U. Whitcher, Zeta functions of alternate mirror Calabi-Yau families, Israel J. Math. 228 (2018), no. 2, 665-705.

[25] B. Dwork, p-adic cycles, Publ. Math. l'IHÉS 37 (1969), no. 1, 27-115.

[26] J.G. Fuselier, L. Long, R. Ramakrishna, H. Swisher, and F.-T. Tu, Hypergeometric functions over finite fields, Preprint arXiv: 1510.02575 [math.NT] (2015); Mem. Amer. Math. Soc. (to appear).

[27] J.G. Fuselier and D. McCarthy, Hypergeometric type identities in the $p$-adic setting and modular forms, Proc. Amer. Math. Soc. 144 (2016), no. 4, 1493-1508.

[28] V.J.W. Guo and W. Zudilin, A q-microscope for supercongruences, Adv. Math. 346 (2019), 329-358.

[29] V.J.W. Guo and W. Zudilin, Dwork-type supercongruences through a creative $q$-microscope, J. Combin. Theory Ser. A 178 (2021), Art. 105362.

[30] F. Gouvêa and N. Yui, Rigid Calabi-Yau threefolds over $\mathbb{Q}$ are modular, Expo. Math. 29 (2011), 142-149.

[31] J. Greene, Hypergeometric functions over finite fields, Trans. Amer. Math. Soc. 301 (1987), no. 1, $77-101$.

[32] B.H. Gross and N. Koblitz, Gauss sums and the p-adic $\Gamma$-function, Ann. of Math. (2) 109 (1979), no. $3,569-581$.

[33] R. Hartshorne, Algebraic geometry, Graduate Texts in Math. 52 (Springer-Verlag, New YorkHeidelberg, 1977).

[34] T. Honda, Formal groups and zeta-functions, Osaka Math. J. 5 (1968), 199-213.

[35] S. Hosono, B.H. Lian, and S.-T. Yau, GKZ-generalized hypergeometric systems in mirror symmetry of Calabi-Yau hypersurfaces, Comm. Math. Phys. 182 (1996), no. 3, 535-577.

[36] A. Huang, B. Lian, S.-T. Yau, and C. Yu, Period integrals of local complete intersections and tautological systems, Preprint arXiv: 1801.01194 [math.NT] (2018).

[37] J.W. Hoffman and F.-T. Tu, Transformations of hypergeometric motives, Preprint arXiv: 2003.05031 [math.NT] (2020).

[38] S.N. Kadir, The arithmetic of Calabi-Yau manifolds and mirror symmetry, DPhil thesis (Oxford University, 2004); Preprint arXiv: hep-th/0409202 (2004).

[39] S. Kadir and N. Yui, Motives and mirror symmetry for Calabi-Yau orbifolds, in: Modular forms and string duality, Fields Inst. Commun. 54 (Amer. Math. Soc., Providence, RI, 2008), 3-46.

[40] N.M. Katz, Exponential sums and differential equations, Ann. Math. Studies 124 (Princeton Univ. Press, Princeton, 1990 
[41] N.M. Katz, Travaux de Dwork, in: Sém. Bourbaki, 24ème année (1971/72), Exp. no. 409, Lecture Notes in Math. 317 (Springer, Berlin, 1973), 167-200.

[42] N.M. Katz, Another look at the Dwork family, in: Algebra, arithmetic, and geometry: In honor of Yu.I. Manin, vol. II, Progr. Math. 270 (Birkhäuser, Boston, MA, 2009), 89-126.

[43] T. Kilbourn, An extension of the Apéry number supercongruence, Acta Arith. 123 (2006), no. 4, 335348.

[44] A. Klemm and S. Theisen, Mirror maps and instanton sums for complete intersections in weighted projective space, Modern Phys. Lett. A 9 (1994), no. 20, 1807-1817.

[45] N. Koblitz, The hypergeometric function with p-adic parameters, in: Proceedings of the Queen's Number Theory Conference, 1979 (Kingston, Ont., 1979), Queen's Papers in Pure and Appl. Math. 54 (Queen's Univ., Kingston, Ont., 1980), 319-328.

[46] N. Koblitz, The number of points on certain families of hypersurfaces over finite fields, Compositio Math. 48 (1983), no. 1, 3-23.

[47] The LMFDB Collaboration, The L-functions and modular forms database, http://www.lmfdb.org (2013-2021); Holomorphic cusp forms, http://www.lmfdb.org/ModularForm/GL2/Q/holomorphic/ (accessed 1 August 2020).

[48] W.C. Li, T. Liu, and L. Long, Potentially $G L_{2}$-type Galois representations associated to noncongruence modular forms, Trans. Amer. Math. Soc. 371 (2019), no. 8, 5341-5377.

[49] W.C. Li, L. Long, and F.-T. Tu, A Whipple ${ }_{7} F_{6}$ formula revisited, La Matematica, accepted in 2021 subject to suggested edits (2021)

[50] A. Libgober and J. Teitelbaum, Lines on Calabi-Yau complete intersections, mirror symmetry, and Picard-Fuchs equations, Intern. Math. Res. Not. (1993), no. 1, 29-39.

[51] L. Long, Hypergeometric evaluation identities and supercongruences, Pacific J. Math. 249 (2011), no. $2,405-418$.

[52] L. Long, Some numeric supercongruences, Contemp. Math. 753 (2020), 139-156.

[53] L. Long, R. Plaza, P. Sin, and Q. Xiang, Characterization of intersecting families of maximum size in PSL(2,q), J. Combin. Theory Ser. A 157 (2018), 461-499.

[54] L. Long and R. Ramakrishna, Some supercongruences occurring in truncated hypergeometric series, Adv. Math. 290 (2016), 773-808.

[55] D. McCarthy, The trace of Frobenius of elliptic curves and the p-adic gamma function, Pacific J. Math. 261 (2013), no. 1, 219-236.

[56] D. McCarthy, Binomial coefficient-harmonic sum identities associated to supercongruences, Integers 11 (2011), A37.

[57] D. McCarthy, On a supercongruence conjecture of Rodriguez-Villegas, Proc. Amer. Math. Soc. 140 (2012), no. 7, 2241-2254.

[58] D. McCarthy, Extending Gaussian hypergeometric series to the p-adic setting, Intern. J. Number Theory 8 (2012), no. 7, 1581-1612.

[59] C. Meyer, A dictionary of modular threefolds, Dissertation (Mainz, 2005).

[60] D. Morrison, Picard-Fuchs equations and mirror maps for hypersurfaces, in: Essays on mirror manifolds (Internat. Press, Hong Kong, 1992), 241-264.

[61] R. Osburn, A. Straub, and W. Zudilin, A modular supercongruence for ${ }_{6} F_{5}$ : an Apéry-like story, Ann. Inst. Fourier (Grenoble) 68 (2018), no. 5, 1987-2004.

[62] A. Peruničić, Introduction to arithmetic mirror symmetry, in: Calabi-Yau varieties: arithmetic, geometry and physics, Fields Inst. Monogr. 34 (Fields Inst. Res. Math. Sci., Toronto, ON, 2015), 503-539.

[63] G. Pólya and G. Szegö, Problems and theorems in analysis, Vol. II, Grundlehren Math. Wiss. 216 (Springer-Verlag, Berlin et al., 1976).

[64] D.P. Roberts and F. Rodriguez Villegas, Hypergeometric supercongruences, in: 2017 MATRIX Annals, MATRIX Book Ser. 2 (Springer, Cham, 2019), 435-439.

[65] D.P. Roberts, F. Rodriguez-Villegas, and M. Watkins, Hypergeometric motives, Preprint (2017-2020).

[66] F. Rodriguez-Villegas, Hypergeometric families of Calabi-Yau manifolds, in: Calabi-Yau varieties and mirror symmetry (Toronto, ON, 2001), Fields Inst. Commun. 38 (Amer. Math. Soc., Providence, RI, 2003), 223-231.

[67] F. Rodriguez-Villegas, Hypergeometric motives, Lecture notes (2017). 
[68] SageMath: Sage Mathematics Software System vers. 9.2 (The Sage Developers, 2020), https://www. sagemath.org.

[69] C. Schoen, On the geometry of a special determinantal hypersurfaces associated to the MumfordHorrocks vector bundle, J. Reine Angew. Math. 364 (1986), 85-111.

[70] J. Stienstra, Formal group laws arising from algebraic varieties, Amer. J. Math. 109 (1987), no. 5, 907-925.

[71] J. Stienstra, Formal groups and congruences for L-functions., Amer. J. Math. 109 (1987), no. 6, 11111127.

[72] J. Stienstra and F. Beukers, On the Picard-Fuchs equation and the formal Brauer group of certain elliptic K3-surfaces, Math. Ann. 271 (1985), no. 2, 269-304.

[73] H. Swisher, On the supercongruence conjectures of van Hamme, Res. Math. Sci. 2 (2015), Art. 18, $21 \mathrm{pp}$.

[74] L. Van Hamme, Some conjectures concerning partial sums of generalized hypergeometric series, in: $p$ adic functional analysis (Nijmegen, 1996), Lecture Notes in Pure and Appl. Math. 192 (Dekker, 1997), 223-236.

[75] E. Viehweg, Rational singularities of higher dimensional schemes, Proc. Amer. Math. Soc. 63 (1977), no. $1,6-8$.

[76] D. Wan, Mirror symmetry for zeta functions, in: Mirrror Symmetry V, AMS/IP Stud. Adv. Math. 38 (Amer. Math. Soc., Providence, RI, 2006), 159-184.

[77] M. Watkins, Hypergeometric motives notes, Preprint http://magma.maths .usyd.edu.au/ watkins/papers/known.pd (2017).

[78] J. Werner and B. van Geemen, New examples of threefolds with $c_{1}=0$, Math. Z. 203 (1990), 211-225.

[79] J.-D. Yu, Variation of the unit root along the Dwork family of Calabi-Yau varieties, Math. Ann. 343 (2009), no. 1, 53-78.

[80] N. Yui, A note on formal groups and zeta functions, J. Reine Angew. Math. 303/304 (1978), 159-169.

[81] N. Yui, Update on the modularity of Calabi-Yau varieties. With an appendix by H. Verrill, in: CalabiYau varieties and mirror symmetry (Toronto, ON, 2001), Fields Inst. Commun. 38 (Amer. Math. Soc., Providence, RI, 2003), 307-362.

[82] O. Zariski, Reduction of the singularities of algebraic three dimensional varieties, Ann. of Math. (2) 45 (1944), 472-542.

[83] W. Zudilin, An elementary proof of Apéry's theorem, Preprint arXiv: math/0202159 [math.NT] (2002); Apéry's theorem. Thirty years after, Intern. J. Math. Computer Sci. 4 (2009), no. 1, 9-19.

Louisiana State University, Baton Rouge, LA 70803, USA

Email address: llong@lsu.edu

Louisiana State University, Baton Rouge, LA 70803, USA

Email address: ftu@lsu.edu

Department of Mathematics and Statistics, Queen's University, Kingston, On, K7L3n6, CANAdA

Email address: yui@queensu.ca

Department of Mathematics, IMAPP, Radboud University, PO Box 9010, 6500 GL NiJMEGEn, The Netherlands

Email address: w.zudilin@math.ru.nl

Laboratory of Mirror Symmetry and Automorphic Forms, National Research University Higher School of Economics, 6 Usacheva str., 119048 Moscow, Russia

Email address: wzudilin@gmail.com 